\title{
PLASMA POL YMERIZED HYDROGEL THIN FILMS FOR APPLICATIONS IN SENSORS AND ACTUATORS
}

\author{
A Dissertation \\ Presented to \\ The Academic Faculty
}

by

Prabhakar A. Tamirisa

\begin{abstract}
In Partial Fulfillment of the Requirements for the Degree

Doctor of Philosophy in the

School of Chemical \& Biomolecular Engineering
\end{abstract}

Georgia Institute of Technology

December 2006 


\section{PLASMA POLYMERIZED HYDROGEL THIN FILMS FOR APPLICATIONS IN SENSORS AND ACTUATORS}

Approved by:

Dr. Dennis W. Hess, Advisor School of Chemical \& Biomolecular Engineering Georgia Institute of Technology

Dr. Clifford L. Henderson School of Chemical \& Biomolecular Engineering Georgia Institute of Technology

Dr. William D. Hunt School of Electrical \& Computer Engineering Georgia Institute of Technology
Dr. J. Carson Meredith

School of Chemical \& Biomolecular Engineering Georgia Institute of Technology

Dr. Mark R. Prausnitz School of Chemical \& Biomolecular Engineering Georgia Institute of Technology 


\section{ACKNOWLEDGEMENTS}

First, I would like to thank my research advisor, Prof. Dennis Hess for providing valuable guidance, financial support, and encouraging creative ideas and critical thinking throughout my stay at Georgia Institute of Technology. I also wish to thank my committee members Prof. Clifford Henderson, Prof. William Hunt, Prof. Carson Meredith, and Prof. Mark Prausnitz for their suggestions, feedback, and critique of my work. I thank Prof. William Hunt and Dr. Sang-Hun Lee, School of Electrical \& Computer Engineering for collaborating on the development of the electrophoretic immobilization procedure and surface acoustic wave sensors. I wish to thank Prof. Clifford Henderson for kindly allowing the use of many analytical instruments. Most of this work would not have been possible without access to the thin film characterization equipment. I would like to thank Prof. Y. C. (Jerry) Jean, Dept. of Chemistry, University of Missouri-Kansas City, for kindly agreeing to conduct positron annihilation studies on the plasma deposited hydrogel films, and assisting in the interpretation of the results. I would also like to thank Prof. Yulin Deng for kindly allowing the use of a contact angle measurement apparatus at the Institute of Paper Science and Technology. I thank Dr. Jere Koskinen, formerly of the Institute of Paper Science and Technology, for collaborating on the initial stages of this project, and providing financial support. I also thank Ms. Gloria Beale for processing numerous purchase orders, Mr. Scott Fowler for helping me troubleshoot the RF power system, and Mr. Johnafel Crowe for training me on the confocal laser scanning microscope and providing several useful suggestions. 
Thanks to Dr. Sudeep Vaswani, former member of Prof. Hess' group, for training me on the operation of the plasma reactors. I would like to acknowledge several members (both former and present) of Prof. Hess' research group for sharing their valuable insights on my experiments during and outside of the group meetings, training me on various analytical instruments, and their camaraderie (in no particular order): Dr. Matthew Spuller, Dr. Ebony Mays, Dr. Jie Diao, Dr. Satyanarayana Myneni, Dr. Christopher Timmons, Dr. Qian Luo, Dr. Galit Levitin, Ashwini Sinha, Lingbo Zhu, Ingu Song, Yonghao Xiu, Gelareh Shakourian, Shantanu Pathak and Bala Balakrishna. I thank Mr. Safdar Ali, for working with me to study the stability of NIPAAm thin films. In addition, I would like to thank Dr. Desmond Stubbs, Mr. Tony Dickherber, and Mr. Christopher Corso, members of the 'maglab', for their valuable comments and support. I would also like to thank Mr. Daniel Hallow for loaning a DC power source.

I also thank Troy, Sean, David, Len, and Murali, for their friendship and support during my stay in Atlanta. Finally, I thank my parents for their love, encouragement, and precious support over the years. 


\section{TABLE OF CONTENTS}

Page

ACKNOWLEDGEMENTS

iii

LIST OF TABLES

vii

LIST OF FIGURES Viii

SUMMARY $\quad$ xi

\section{CHAPTER}

1 INTRODUCTION 1

2 BACKGROUND 5

2.1 Plasma polymerization $\quad 5$

$\begin{array}{ll}2.2 \text { Hydrogels } & 15\end{array}$

$\begin{array}{ll}2.3 \text { Applications in sensors and actuators } & 21\end{array}$

3 SYNTHESIS AND CHARACTERIZATION 29

$\begin{array}{ll}3.1 \text { Introduction } & 29\end{array}$

3.2 Experimental Section 31

3.3 Results and Discussion 36

3.3.1 Infrared Spectroscopy Characterization of Hydrogel Films $\quad 38$

3.3.2 Thermoresponsive Properties of Hydrogel Films 44

3.4 Conclusions $\quad 49$

4 WATER AND MOISTURE UPTAKE CHARACTERISTICS 51

$\begin{array}{ll}4.1 \text { Introduction } & 51\end{array}$

4.2 Experimental Section $\quad 55$

$\begin{array}{ll}\text { 4.3 Results and Discussion } & 58\end{array}$ 
4.4 Conclusions

5 MICROSTRUCTURE CHARACTERIZATION

5.1 Introduction

5.2 Experimental Section

5.3 Results and Discussion

5.4 Conclusions

6 SUMMARY AND RECOMMENDATIONS

6.1 Summary

6.2 Recommendations

APPENDIX A: ELECTROPHORETIC IMMOBILIZATION AND SENSOR DEVELOPMENT

APPENDIX B: STABILITY STUDIES 


\section{LIST OF TABLES}

Page

Table 2.1: Examples of polymers that exhibit LCST behavior in aqueous solutions ${ }^{1} 16$

Table 3.1: VASE results of NIPAAm films deposited on silicon 37

Table 3.2: $\quad$ FTIR band assignments for NIPAAm films deposited on silicon 41

Table 5.1: Thickness and refractive index of plasma polymerized NIPAAm films from $\begin{array}{ll}\text { variable angle spectroscopic ellipsometry } & 81\end{array}$

Table 5.2: $\quad$ Fitted parameters (from VEPFIT $^{2}$ ) for DBES plots shown in Figure 5.1

Table B.1: Thickness values of NIPAAm films exposed to DI water

Table B.2: Thickness values of NIPAAm films exposed to PBS

Table B.3: Thickness values of NIPAAm films exposed to TAE

Table B.4: Comparison of Amide I and II bands in the FTIR spectra 


\section{LIST OF FIGURES}

Page

Figure 2.1: Schematic of the competitive ablation and polymerization (CAP) mechanismof plasma polymerization: (1) Ablation of the monomer to form reactive species in the plasma phase; (2) Adsorption of reactive species on substrate; (3) Heterogeneous reaction with substrate to form plasma polymer film; (4) Desorption of unreacted species from substrate ${ }^{25}$

Figure 2.2: Relationship between basic plasma parameters and plasma-surface interaction parameters ${ }^{3}$

Figure 3.1: Schematic of the capacitively coupled, parallel plate plasma reactor used to deposit NIPAAm thin films

Figure 3.2: FTIR spectra of NIPAAm films deposited on silicon at $133.3 \mathrm{~Pa}$ at $150{ }^{\circ} \mathrm{C}$, $175^{\circ} \mathrm{C}$ and $200{ }^{\circ} \mathrm{C} ; 30 \mathrm{~W}$ rf power

Figure 3.3: FTIR spectra of NIPAAm films deposited on silicon at $93.3 \mathrm{~Pa}$ at $120^{\circ} \mathrm{C}$, $150{ }^{\circ} \mathrm{C}, 175^{\circ} \mathrm{C}$ and $200{ }^{\circ} \mathrm{C} ; 30 \mathrm{~W}$ rf power

Figure 3.4: Contact angles of plasma polymerized NIPAAm on silicon surface deposited at (a) $120{ }^{\circ} \mathrm{C}, 93.3 \mathrm{~Pa}$, (b) $150{ }^{\circ} \mathrm{C}, 133.3 \mathrm{~Pa}$. RF power was $30 \mathrm{~W}$ for all samples, (c) $150{ }^{\circ} \mathrm{C}, 93.3 \mathrm{~Pa}$, and (d) $175^{\circ} \mathrm{C}, 133.3 \mathrm{~Pa}$. RF power was $30 \mathrm{~W}$ for all samples

Figure 4.1: Variation of $-\Delta \mathrm{f}$ (top) and $\Delta \mathrm{D}$ (bottom) with temperature for NIPAAm films prepared using process condition $\mathrm{B}$ (Substrate temperature $=120^{\circ} \mathrm{C}$; Reactor pressure $=93.3 \mathrm{~Pa}$ )

Figure 4.2: Variation of $-\Delta \mathrm{f}$ (top) and $\Delta \mathrm{D}$ (bottom) with temperature for NIPAAm films prepared using process condition $\mathrm{C}$ (Substrate temperature $=175^{\circ} \mathrm{C}$; Reactor pressure $=133.3 \mathrm{~Pa}$ )

Figure 4.3: Variation of $-\Delta \mathrm{f}$ (top) and $\Delta \mathrm{D}$ (bottom) with temperature for NIPAAm films prepared using process condition $\mathrm{D}$ (Substrate temperature $=175^{\circ} \mathrm{C}$; Reactor pressure $=93.3 \mathrm{~Pa}$ )

Figure 4.4: Variation of $-\Delta \mathrm{D} / \Delta \mathrm{f}(1 \mathrm{E}-6)$ with temperature for NIPAAm films prepared using different reactor conditions

Figure 4.5: Moisture absorption kinetics from ambient air ( $\sim 40 \%$ relative humidity) for NIPAAm film prepared using: (a) process condition B (Substrate temperature $=120^{\circ} \mathrm{C}$; Reactor pressure $=93.3 \mathrm{~Pa}$ ); (b) process condition $\mathrm{C}$ 
(Substrate temperature $=175^{\circ} \mathrm{C}$; Reactor pressure $=133.3 \mathrm{~Pa}-$ top $) ;(\mathrm{c})$ process condition $\mathrm{D}\left(\right.$ Substrate temperature $=175^{\circ} \mathrm{C}$; Reactor pressure $=$ 133.3 $\mathrm{Pa}$ - bottom)

Figure 4.6: Comparison of moisture uptake capacities of NIPAAm films prepared using different reactor conditions, through swelling ratio, $\mathrm{Q}$

Figure 5.1: Interactions of positron and positronium with condensed matter ${ }^{4}$

Figure 5.2: A schematic diagram of the slow positron beam at the University of Missouri-Kansas City. A: $50 \mathrm{mCi}{ }^{22} \mathrm{Na}$ positron source, B: W-mesh moderator, C: magnetic field ( $75 \mathrm{G})$ coils, D: E X B filter, E: positron accelerator and electric insulator, F: correcting magnets, G: gas inlet, $\mathrm{H}$ : positron lifeline system for PAL, I: turbo molecular pump, J: samples, K: sample manipulator, L: cryo pump, M: Ge solid state detector, N: PAL detector $^{5}$

Figure 5.3: Variation of S parameter as a function of positron incident energy in plasma polymerized NIPAAm thin films prepared under different process conditions: (a) $0<$ positron incident energy $<30 \mathrm{keV}$ (top) (b) $0<$ positrons incident energy $<5 \mathrm{keV}$ (bottom)

Figure 5.4: Values of S parameter of the second layer and refractive index of plasma polymerized NIPAAm films prepared under different reactor conditions

Figure A.1: Schematic of the electrophoretic immobilization set up (not drawn to scale)

Figure A.2: Frequency response of SAW devices after NIPAAm deposition (---), after anti-FITC immobilization (---), and after exposure to uranine vapor (---); (a) Electrophoretic immobilization at $5 \mathrm{~V}$ (top); (b) Electrophoretic immobilization at $1 \mathrm{~V}$ (bottom); (c) No anti-FITC immobilization

Figure A.3: Frequency response of SAW devices after NIPAAm deposition (---), after anti-FITC immobilization (---), and after exposure to uranine vapor (---); (a) Electrophoretic immobilization at $0.5 \mathrm{~V}$ for $15 \mathrm{~min}$ (top); (b) Electrophoretic immobilization at $0.5 \mathrm{~V}$ for $10 \mathrm{~min}$ (bottom); (c) NIPAAm film - no electrophoretic immobilization (top); (d) Blank SAW device - No NIPAAm film, no electrophoretic immobilization (bottom)

Figure A.4: CLSM images: (a) Anti-FITC containing NIPAAm film ( $0.5 \mathrm{~V}$ for $15 \mathrm{~min})$; (b) Anti-FITC containing NIPAAm film (0.5 V for $10 \mathrm{~min})$; (c) Blank SAW device - no hydrogel film; (d) Hydrogel coated SAW device - no anti-FITC 
Figure B.1: Thickness of NIPAAm films prepared under different reactor conditions as a function of exposure to DI water

Figure B.2: Thickness of NIPAAm films prepared under different reactor conditions as a function of exposure to PBS solution

Figure B.3: Thickness of NIPAAm films prepared under different reactor conditions as a function of exposure to TAE solution

Figure B.4: FTIR spectra of NIPAAm films exposed to DI water showing the amide I and II regions

Figure B.5: FTIR spectra of NIPAAm films exposed to PBS solution showing the amide I and II regions

Figure B.6: FTIR spectra of NIPAAm films exposed to TAE solution showing the amide I and II regions 


\section{SUMMARY}

Plasma polymerization was used to produce thermoresponsive, hydrogel films of NIsopropylacrylamide (NIPAAm) in a single step. Through variation of reactor conditions such as deposition pressure and substrate temperature, physicochemical properties of the hydrogel films such as crosslink density and thus swelling could be controlled. Chemical bonding structures in plasma polymerized NIPAAm were studied using Fourier transform infrared spectroscopy (FTIR). Contact angle goniometry and quartz crystal microbalance with dissipation monitoring were used to confirm the existence of a hydrophilichydrophobic transition in plasma polymerized NIPAAm thin films, analogous to the lower critical solution temperature (LCST) transition in linear, uncrosslinked chains. Hydrogen bonding in NIPAAm thin films was found to control the moisture uptake capacity; films prepared at higher substrate temperatures and lower reactor pressures, and hence believed to possess greater crosslink density, showed the highest moisture uptake capacity in ambient humidity. Free volume characteristics of NIPAAm thin films were studied using Doppler broadening energy spectroscopy (DBES). Furthermore, a novel, electrophoretic procedure was conceived to incorporate biomolecules such as antibodies in plasma polymerized NIPAAm films for use as sensing layers in vapor phase, surface acoustic wave sensors. 


\section{CHAPTER 1}

\section{INTRODUCTION}

Hydrogels, an important class of soft materials, are water swollen polymeric networks that are capable of absorbing large quantities of water while retaining their physical integrity. "Intelligent" hydrogels respond to environmental triggers such as $\mathrm{pH}^{6}$, temperature ${ }^{7}$, and electric fields ${ }^{8}$ to undergo volume changes wherein the polymeric network expands upon absorption of water or collapses by releasing the absorbed water. Temperature-sensitive hydrogels such as poly(N-Isopropylacrylamide) (NIPAAm) undergo a volume phase transition analogous to the lower critical solution temperature (LCST) transition in linear chains; below the transition temperature, the hydrogel absorbs water and expands, whereas above the transition temperature, the hydrogel collapses and releases the absorbed water. Hydrogels have been used in a variety of applications such as drug delivery ${ }^{9,10}$, bioseparations ${ }^{11}$, sensors ${ }^{1}$, intelligent surfaces $^{12}$, and tissue culture ${ }^{13}$, 14.

The physicochemical properties of hydrogels, which determine their suitability for various applications, are established by the processing technique and parameters used to create them. In bulk hydrogels, crosslink density is an important parameter that controls the mechanical properties and water uptake capacity, and can be varied to obtain desired properties. Furthermore, the properties of bulk, thermoresponsive gels may be modeled through various thermodynamic and transport theories, thus allowing a greater 
understanding of the structure-property correlations. However, knowledge of bulk hydrogel behavior may not be useful when applied to structures used in various applications where the hydrogel may be constrained in some way. For example, in the case of thin films of thermoresponsive, poly (NIPAAm) produced by techniques such as surface grafting ${ }^{15,16}$, photopolymerization ${ }^{17,18}$ and plasma polymerization ${ }^{19-21}$, the phase transition behavior is significantly different relative to bulk hydrogels, and is believed to be the result of the constraining substrate. In the thin film geometry, the hydrogel is unable to expand or contract in the lateral directions, thus confining the motion to a single direction, normal to the substrate.

In contrast to the conventional methods used to produce hydrogel thin films, plasma polymerization is a solution-free approach to prepare crosslinked organic thin films in a single step. During plasma polymerization, well-adhering, crosslinked polymer films are deposited directly on a substrate; subsequent exposure of the polymer films to water results in the formation of a hydrogel structure and removal of uncrosslinked polymeric segments. In this work, plasma polymerized NIPAAm thin films have been prepared to study the effect of plasma processing parameters such as substrate temperature and reactor operating pressure on the physicochemical properties of the NIPAAm films. The motivation for this study is derived from the understanding that by varying process conditions, it is possible to alter the crosslink density of the hydrogel network in a controlled manner, and thus produce hydrogel films of desired properties. 
While plasma polymerized NIPAAm thin films have been prepared by other investigators ${ }^{19,20}$, details of the correlation between processing conditions and thin film properties are not available. Furthermore, to the author's knowledge, no attempts were made to vary the crosslink density by controlling the temperature of the growing film or the reactor pressure. The synthesis of plasma polymerized NIPAAm films in a radio frequency, parallel plate plasma reactor and investigation of the thin film properties through variable angle spectroscopic ellipsometry (VASE), Fourier transform infrared (FTIR) spectroscopy, and temperature dependent contact angle measurements are described in Chapter 3. Water and moisture uptake studies of the plasma polymerized NIPAAm films are given in Chapter 4, whereas details of a preliminary investigation into the microstructure of NIPAAm films through positron annihilation lifetime spectroscopy are given in Chapter 6.

In addition, a novel electrophoretic immobilization procedure was conceived to prepare biomolecule sensitive hydrogel thin films. Preliminary experiments were conducted to drift charged biomolecules such as antibodies into plasma polymerized NIPAAm films to use the resulting plasma polymer-biomolecule composite as an immunosensing layer on acoustoelectronic transducers such as quartz crystal microbalances and surface acoustic wave devices. The post deposition incorporation of biomolecules into plasma polymerized films presents significant advantages from the standpoint of large scale production of sensors. First, both plasma polymerization and the electrophoretic incorporation procedure are suitable for large scale production. If the electrophoretic procedure is used, chemical derivatization reagents and procedures, 
typically invoked in conjugating biomolecules to hydrogel films may be avoided.

Furthermore, since the biomolecules are immobilized in the hydrogel matrix in a threedimensional approach rather than only on the surface, greater density of molecular recognition sites should be achieved, thereby increasing the sensitivity of detection. Details of the electrophoretic incorporation procedure and sensor development are given in Appendix A. The stability of plasma polymerized NIPAAm films was investigated through prolonged exposure to deionized water, phosphate buffered saline, and tris acetate buffer. The physicochemical properties of plasma polymerized NIPAAm thin films were studied using VASE and FTIR spectroscopy as a function of exposure; results from these studies are given in Appendix B. 


\section{CHAPTER 2}

\section{BACKGROUND}

\subsection{Plasma polymerization}

Plasmas have often been referred to as the "fourth state of matter" or an "ionized gas", and can be broadly defined as a collection of oppositely charged carriers in the vapor phase with a net zero electric charge. Plasmas can be classified into two broad categories based on the degree of ionization and energy levels of the species constituting them: hot (equilibrium) plasmas and cold (non-equilibrium) plasmas. Plasma polymerization and other types of materials processing involving plasmas are typically carried out using electrically driven, low-pressure plasmas of the latter type, which are characterized by low degrees of ionization, and lower energies than the hot plasmas. Since the energy of the ionized atoms or molecules in such plasmas is lower than that of the electrons, these particle types are not in thermal equilibrium with each other. Furthermore, the electrons are not in equilibrium with the surrounding gas.

Consequently, the plasma does not heat the substrate or the surrounding reactor walls substantially $\left(<100^{\circ} \mathrm{C}\right)$, and hence is referred to as a cold plasma ${ }^{22}$.

Plasma polymerization refers to the formation of thin films from plasmas of organic precursors or monomers. Energetic species such as free radicals, ions, electrons, and excited state species, which are formed in the plasma by electron impact collisions causing ionization and fragmentation of the organic precursors, react with other energetic species 
and with the surface of a substrate to form a polymer film. Since plasma-deposited polymers are formed through chemical reactions at surface sites in a radiation environment, the resulting films do not resemble conventional polymers that contain a single repeat unit. Rather, these materials are crosslinked as deposited, are pinhole free and homogeneous, have excellent adhesion to substrates or other films, are chemically and mechanically stable, and do not require the use of solvents for film formation, resulting in a more environmentally benign process. Thus, plasma deposition techniques are attractive alternatives to spin-casting methods and can be used to deposit sensing layers for mass production of sensors based on a variety of transducers.

The monomers that have been used in the plasma polymerization process to date can be broadly grouped under the following categories ${ }^{23}$ :

- Hydrocarbons: This class can further be divided into 1) alkynes and aromatic compounds 2) alkenes, double bond containing and cyclic structures 3) saturated monomers.

- Hydrocarbons containing polar groups: Allylamine, vinyl pyridine and pyridine are examples of such monomers.

- Fluorocarbons

- Silicon containing monomers: Includes linear and cyclic siloxanes and silazanes and silanes.

- Metal containing monomers: Plasma polymers formed from such monomers are usually metal containing polymer composites. High vapor pressure organometallic compounds are the most common monomers of this class. 
Plasma polymerization involves both homogeneous and heterogeneous processes, and several models have been proposed for the complex set of reactive processes. The most popular mechanisms describe a competitive ablation and polymerization mechanism $(\mathrm{CAP})^{24,25}$ (Figure 2.1). During plasma polymerization, monomers undergo fragmentation and polymerization; however depending on the process parameters and reactant species, ablation (resulting in sputtering), chemical etching or polymerization can dominate. For example, fluorocarbon plasmas have been used to etch silicon and also to deposit fluorocarbon polymers on silicon wafers; by changing the process conditions it is possible to perform (net) etching or deposition. Ablation or fragmentation is unique to plasma polymerization, and allows monomers that cannot be conventionally polymerized due to the lack of reactive groups, to be polymerized in the plasma state. However ablation also causes crosslinking in plasma polymers, and hence the resulting polymer is a network consisting of oligomers and unreacted monomers.

It is well known that free radicals are among the most important active species in the plasma polymerization process. Two distinct types of polymerization based on free radicals are known ${ }^{24}$ : plasma-induced polymerization and plasma state polymerization. The former resembles free radical induced polymerization of monomers containing unsaturated carbon-carbon bonds, and requires the plasma only to create the reactive species such as free radicals and electrons required for initiation of polymerization. Following initiation, propagation and termination of polymerization can occur in the 
absence of plasma. On the other hand, in plasma state polymerization, the entire polymerization process, including initiation, propagation and termination occurs in the

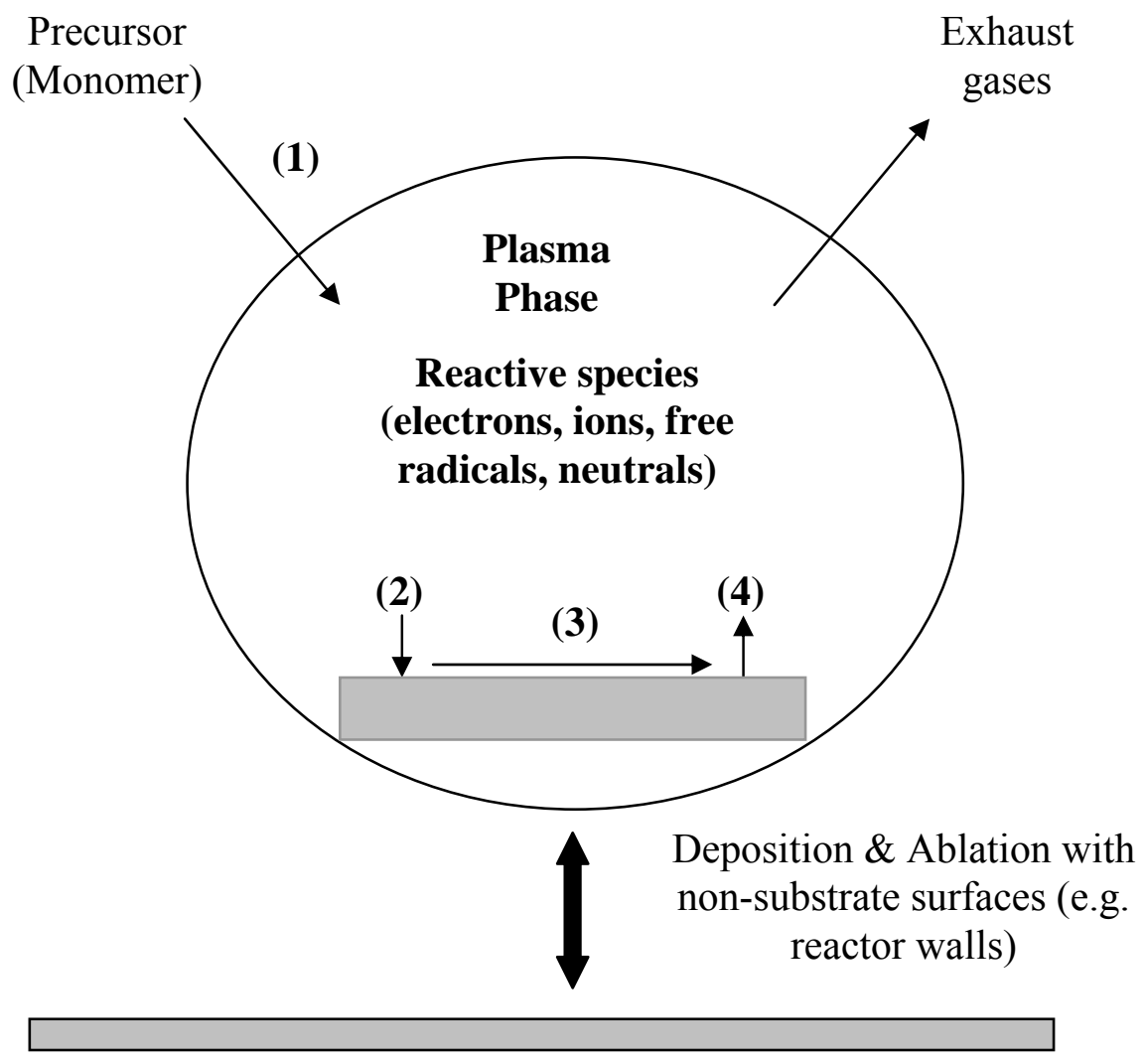

Figure 2.1 Schematic of the competitive ablation and polymerization (CAP) mechanism of plasma polymerization: (1) Ablation of the monomer to form reactive species in the plasma phase; (2) Adsorption of reactive species on substrate; (3) Heterogeneous reaction with substrate to form plasma polymer film; (4) Desorption of unreacted species from substrate $^{25}$.

plasma phase. Since the energy transferred to a monomer in the plasma state is sufficient to create several reactive species either through fragmentation or a simpler rearrangement of atoms, it is possible to polymerize almost any type of monomer, even ones that would not polymerize in the solution phase due to a lack of reactive groups such as vinylic 
double bonds. Furthermore, since the free electrons and radicals in the plasma state are able to react with partially or fully fragmented monomer species, the resultant polymer does not resemble polymers produced from other conventional polymerization processes that proceed via a sequential addition of a single type of repeat unit to an ever-growing macromolecular chain. The presence and impingement of many different types of energetic species promote cross-linking in the polymers. It is believed that the plasma polymer grows through a rapid step growth mechanism ${ }^{25}$.

The term plasma polymer of a certain monomer refers to not one macromolecule but a family of macromolecules or networks that can be formed from the same monomer under different plasma processing conditions. In other words, it is possible to produce macromolecular networks with very different properties from the same monomer or precursor just by changing one or several process conditions, and thus basic plasma parameters. The different materials formed could only be predicted a priori if the specific chemical reactions that produce the various structures were known.

The properties of plasma polymers are determined by various factors, which can be classified as: 1) Basic plasma parameters, and 2) Plasma-surface interactions ${ }^{3}$. The basic plasma parameters are: $\mathrm{n}_{\mathrm{e}}$, electron density; $f(E)$, electron energy distribution; $\mathrm{N}$, gas density; $\tau$, residence time of gas molecule in the plasma. Process conditions such as plasma excitation power, excitation frequency, nature of discharge gas or mixture (in the present case, this refers to the type of monomer), gas flow rate, pumping speed, and geometrical factors of the reactor influence the basic plasma parameters directly, whereas 
geometrical factors related to the surface of the substrate, physical and chemical nature of the surface, temperature and potential of surfaces in contact with the plasma affect the plasma-surface interactions and therefore indirectly influence the basic plasma parameters. By varying either the basic plasma parameters through the process conditions or the plasma-surface interactions, it is possible to tailor the properties of the plasma polymerized films (Figure 2.1). A detailed account of the effect of various process parameters on the properties of plasma polymers may be found in the literature ${ }^{23}$.

In this work, the effect of reactor operating pressure and substrate temperature on the physicochemical properties of plasma polymerized hydrogel films of NIsopropylacrylamide has been studied. Specifically, it is believed that the crosslink density, a key parameter in hydrogel films, may be tailored by varying the process conditions. The effect of varying reactor operating pressure may be understood through the changes in the average electron energy, which is inversely proportional to the pressure. Therefore, at lower pressures when the average electron energy is higher, greater fragmentation of the monomer and hence, greater crosslinking may be expected. In contrast, at higher pressures, due to a smaller degree of fragmentation at lower electron temperatures, less crosslinking and hence, more linear chain type of polymers may be expected. 


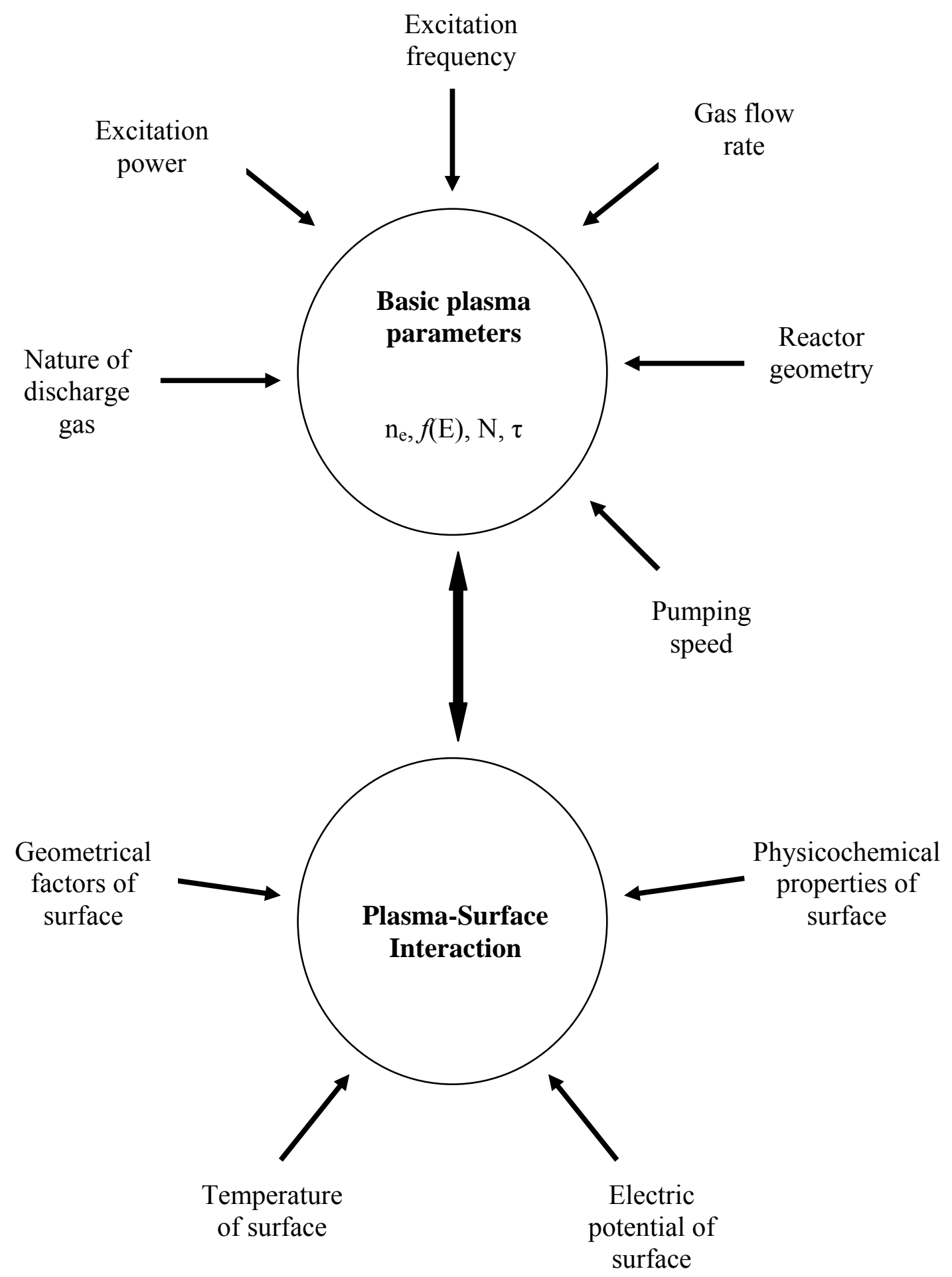

Figure 2.2 Relationship between basic plasma parameters and plasma-surface interaction parameters $^{3}$. 
Substrate temperature is another key parameter used to control the structure of plasma polymers; at higher substrate temperatures, fragmentation and crosslinking of adsorbed species is facilitated relative to polymerization. In a study of the chemical bonding characteristics of plasma polymer films deposited at different substrate temperatures, polymer films formed at lower substrate temperatures contained a greater amount of the intact monomer and presumably a lower level of crosslinking; at higher substrate temperatures due to greater fragmentation, the monomer structure was not preserved $^{26}$. Furthermore, plasma polymer deposition rate decreases with increasing substrate temperature, indicating that plasma polymerization is an adsorption limited process $^{26,27}$. In an attempt to preserve the monomer structure by incorporating slightly modified monomers and oligomers in the plasma polymer film, cryogenically cooled substrates have been used ${ }^{23}$. In a different report, the crosslink density of a plasma polymer formed of two monomers (iron pentacarbonyl and 4-vinyl pyridine) was altered by varying the substrate temperature between 180 and $300{ }^{\circ} \mathrm{C}$. The electrochemical response of the resulting plasma polymer films depended on the crosslink density; charge transfer between redox centers was facilitated in less crosslinked films relative to highly crosslinked films ${ }^{28}$.

In addition, composite parameters based on the excitation power (W), precursor flow rate $(F)$, molecular weight $(\mathrm{M})$, and reactor operating pressure $(\mathrm{p})$ have been used to predict the degree of crosslinking, fragmentation, and preservation of the monomer structure. Since the extent of crosslinking is directly proportional to $\mathrm{W}$, and varies inversely with $\mathrm{F}, \mathrm{M}$, and $\mathrm{p}$, three types of parameters have been proposed in the literature: 
$\mathrm{W} / \mathrm{F} \mathrm{M} ; \mathrm{W} / \mathrm{F}^{24}$; and $\mathrm{W} / \mathrm{F} \log (\mathrm{p})^{29}$; clearly lower values of the parameters are associated with greater retention of the monomer structure and a smaller degree of crosslink density. The degree of crosslinking in plasma polymer films has been inferred by analyzing the physicochemical properties through $\mathrm{x}$-ray photoelectron spectroscopy $(\mathrm{XPS})^{30}$, Fourier transform infrared spectroscopy $(\mathrm{FTIR})^{31}$, mechanical properties through nanoindentation ${ }^{32}$, solvent swelling measurements through neutron reflectivity ${ }^{33}$, and density inhomogeneities through small angle x-ray scattering ${ }^{34}$.

Different types of plasma sources have used for plasma polymerization. The oldest and best-documented source for plasma polymerization is perhaps the $\mathrm{DC}$ or $\mathrm{AC}$ (up to $50 \mathrm{kHz}$ ) glow discharge ${ }^{35}$, which has been used in a bell jar type of reactor fitted with parallel plate electrodes. Another common source operates in the radio frequency $(\mathrm{RF})$ regime $(100 \mathrm{kHz}-30 \mathrm{MHz})$, and is most often used in a tubular reactor with external coils or ring electrodes, therefore relying on a combination of capacitive and inductive coupling of the energy to the plasma. RF energy may also be capacitively coupled to two electrodes placed parallel to each other inside the reactor, in an RF diode type of configuration. More recently, microwave discharges and magnetic field containing discharges like electron cyclotron resonance (ECR) and planar or cylindrical magnetrons are being employed in plasma polymerization ${ }^{23,24}$. Since microwave discharges possess higher energy electrons, due to a higher proportion of electrons in the high energy tail of the electron energy distribution, energy is coupled to the discharge more efficiently than in lower frequency discharges. Consequently, greater fragmentation of the monomer is expected, leading to highly crosslinked polymer films. 
Crosslink density of plasma polymers may also be modulated through pulsed plasma polymerization, which can be accomplished by pulsing an RF discharge in a plasma reactor. Pulsing the RF field is known to decrease the concentration of free radicals by almost ten fold ${ }^{36}$, which ensures greater control of the film chemistry ${ }^{37,38}$ because reduction in the average electron concentration and electric field reduces the possibility of various fragmentation reactions occurring. Furthermore, a smaller free radical concentration during the polymerization results in a lower free radical concentration in the deposited film, and thus reduces the possibility of forming "living polymers" 24 . Film chemistry from pulsed plasma polymerization experiments has been studied as a function of the ratio of the "on" time to the "off" time by several authors" Pulsed plasma polymerization can be particularly useful with aromatic monomers because these compounds are known to produce an unusually large number of free radicals with other plasma polymerization methods. A decrease in the density of free radicals in the plasma can have a favorable effect on the retention of the original monomer structure and control of film chemistry. Since pulsed plasmas apply a lower average power to the monomer vapor, the average deposition (polymerization) rate is lower relative to that of continuous wave plasmas.

This section is intended as a brief introduction to the topic of plasma polymerization. The reader is referred to reviews and monographs dealing with plasma polymerization for a more extensive coverage of the subject ${ }^{23,24,35,42}$. 


\section{$2.2 \quad$ Hydrogels}

Hydrogels are water-swollen crosslinked polymeric structures derived from hydrophilic monomers. They are prepared by the polymerization of one or more monomers, and are crosslinked in a variety of ways: radiation crosslinking ${ }^{43} 44$ through the use of electron beams, $\mathrm{x}$-rays, ultra-violet light, gamma rays; covalent crosslinking through the use of a chemical crosslinking agent, which is at least difunctional; physical crosslinking through various intermolecular forces such as hydrogen bonds, and strong van der Waals interactions between polymeric chains ${ }^{45}$. The hydrophilic nature of individual monomers allows absorption of water, whereas the crosslinked network-like structure of hydrogels prevents dissolution in water. Hydrogels prepared using the methods described above are generally synthesized in aqueous solutions, thereby introducing a considerable amount of water in the hydrogel networks during crosslinking. Furthermore, formation of hydrogel thin films using solution based methods involves an additional step of spincoating or dip-coating. In contrast, plasma polymerization allows the production of highly networked thin films of hydrophilic monomers from the vapor phase in a single step; hydration of the hydrophilic, thin film networks after deposition results in the formation of a hydrogel film.

"Intelligent" hydrogels respond to a variety of environmental stimuli such as $\mathrm{pH}^{6}$, temperature ${ }^{46}$, electric field ${ }^{8}$, light $^{47}$ and stress ${ }^{48}$ through volume or conformation changes. The macroscopic changes are a result of fast, reversible changes at the 
microscopic level and have led to novel applications in drug delivery ${ }^{9,10}$, bioseparations ${ }^{11}$,

Table 2.1 Examples of polymers that exhibit LCST behavior in aqueous solutions ${ }^{1}$

\section{Amide groups}

Poly(N-substituted acrylamides)

Poly(N-acryloyl pyrrolidine)

Poly(N-acryloyl piperidine)

Poly(acryl-L-amino acid amides)

Poly(vinyl lactams)

\section{Ether groups}

Poly(vinyl methyl ether)

Poly(EO/PO) random copolymers

PEO-PPO-PEO triblock surfactants

Alkyl-PEO block surfactants

\section{Alcohol groups}

Hydroxypropyl acrylate

Hydroxypropyl methylcellulose

Hydroxypropyl cellulose

Methylcellulose

Poly(vinyl alcohol) derivatives

\section{Other}

Poly(methacrylic acid) 
sensors $^{1}$, and intelligent surfaces ${ }^{12}$. Temperature sensitive hydrogels may be prepared from monomers and linear polymers that exhibit inverse temperature solubility in aqueous solutions as a result of a phase transition induced by a lower critical solution temperature (LCST) or cloud point (CP). A variety of polymers demonstrate the thermally-induced LCST or CP transition (Table I). Thermoresponsive hydrogels based on N-Isopropylacrylamide (NIPAAm) have been the most extensively studied, and demonstrate thermoshrinking or LCST behavior: a hydrophilic and swollen state at temperatures below LCST and a hydrophobic and collapsed state above LCST. Immobilizing thermoshrinking/thermoresponsive hydrogels in the form of thin films on a solid support such as a silicon wafer allows expansion and contraction essentially only in the direction normal to the substrate. The resulting temperature-sensitive hydrogel structure offers the potential for sensor and actuator applications.

Numerous mathematical models have been developed in order to understand the phenomenon of volume phase transitions in hydrogels ${ }^{49-53}$. Models describing volume transitions in hydrogels may be broadly classified into two types: thermodynamic models and transport models ${ }^{54}$.

Thermodynamic models are the most popular and use free energy or chemical potential or the related osmotic pressure to describe the equilibrium state of hydrogels. The assumption of four different contributions to the free energy arising from mixing, elasticity, ionic species and other specific interactions for a gel and the aqueous media is 
central to all gel transition theories. There is little disagreement on the description of osmotic pressure due to ionic species in the gel: Donnan equilibrium is consistently assumed. However, mathematical expressions for the three other contributions to free energy vary from theory to theory. For example, use of Flory-Huggins theory for the free energy of mixing presents a simple and perhaps incorrect picture of the gel in aqueous media since it assumes random placement of species and that the lattice is incompressible. The elasticity term is commonly described by rubber elasticity theory, which assumes Gaussian chains and does not account for the chains being finite. Free energy expressions from specific interactions such as hydrogen bonding or hydrophobic bonds are not commonly invoked and the additivity of such terms with the other three terms is often questioned, since the effect of specific interactions need to be accounted for in the other three terms also 54 .

While certain theories are able to model and predict the experimental results quite well, they are not universal; some are unable to predict a continuous / discontinuous transition or a pressure dependence of the transition. Furthermore, use of the thermodynamic models to predict phase behavior of hydrogels is inhibited when the various thermodynamic parameters are unknown. Extension of thermodynamic theories developed for bulk hydrogels to hydrogel thin films is not common mainly because the effect of the constraint imposed by the substrate on hydrogel properties is unknown. In one case, the effect of the substrate has been modeled as an additional hydrostatic pressure, but the model is limited since it has been unable to predict conditions and cause for a continuous transition, which is commonly observed in thin films ${ }^{55}$ 
Another major disadvantage of thermodynamic models is that they are capable of predicting equilibrium situations only; information on chain dynamics or diffusion of molecules through the gel network cannot be described. Transport (transient) models assume that the transition of hydrogels is diffusion driven. Hydrogel volume transitions are controlled by hydrogel network stress, electrical potential and osmotic pressure, which are determined by ion diffusive fluxes. Modern transport theories for gels have their origins in the theory of Tanaka, Hocker and Benedeck (THB) ${ }^{56}$, which relates network displacement to existing stress through a solvent-polymer friction coefficient. More recently, continuum approaches for simple mixtures of two ${ }^{57}$ or three ${ }^{58}$ components or multicomponent ${ }^{59}$ mixtures assuming Fickian and non-Fickian diffusion have been developed. The parameters needed for transport models mainly include diffusion coefficients of the mobile species and the elastic parameters of the hydrogels. Elastic parameters of hydrogels are size dependent and can vary during swelling and transitions. However, the transport models are able to describe both the transient and equilibrium states of the hydrogel and are a substantial improvement over thermodynamic models.

As discussed earlier, plasma polymerization is an attractive method to prepare hydrogel thin films because polymerization, crosslinking, and thin film deposition are combined into a single process. Furthermore, the films can be prepared on any substrate with good adhesion, and it is possible to control the crosslink density, an important parameter that affects the physicochemical properties of hydrogels, by varying the process conditions. Early reports on plasma-assisted methods for the production of 
hydrogel films focused on developing cell and protein resistant/non-fouling surfaces for biomedical applications. Monomers such as triethylene glycol monoallyl ether ${ }^{60}, 2-$ hydroxyethyl methacrylate ${ }^{61-63}$, crown ethers ${ }^{64,65}$, oligoglymes ${ }^{26,64,66,67}$, and ethylene oxide containing monomers ${ }^{68,69}$ were plasma polymerized to produce non-stimuliresponsive hydrogel thin films, which resisted protein adsorption.

To date, only a few studies have been reported on the formation of stimuliresponsive hydrogel films through plasma polymerization, and they have all been focused on thermoresponsive films based on NIPAAm ${ }^{13,19,20,70,71}$. The plasma polymerized NIPAAm thin films were characterized using atomic force microscopy (AFM), contact angle measurements, Fourier transform infrared spectroscopy (FTIR), x-ray photoelectron spectroscopy (XPS), time-of-flight secondary ion mass spectroscopy (ToFSIMS), sum frequency generation (SFG) vibrational spectroscopy, and fluorescent protein adsorption studies. Results from these studies generated insight into the physicochemical properties, thermodynamics of the LCST transition, and allowed comparison of properties of plasma polymerized NIPAAm to those of solution polymerized NIPAAm ${ }^{19,20,71}$. In addition, plasma polymerized NIPAAm films were used to develop 'smart' tissue culture substrates ${ }^{13,70}$. Since protein mediated cell adhesion is favored by hydrophobic surfaces relative to hydrophilic surfaces, at physiological temperature $\left(\sim 37^{\circ} \mathrm{C}\right)$, NIPAAm surfaces allow cell adhesion since NIPAAm is above the LCST temperature $\left(\sim 32^{\circ} \mathrm{C}\right)$; however, below the LCST temperature, the cells delaminate as a confluent sheet. In contrast, cells cultured on traditional substrates such as tissue culture polystyrene (TCPS) require chemical or 
mechanical means of removal. Temperature mediated cell-adhesion was first reported several years ago ${ }^{14}$, and has been subsequently studied by several investigators ${ }^{13,72-77}$.

In all previous studies of plasma polymerized NIPAAm, similar plasma reactors, consisting of a copper coil wound around a glass/quartz tube, were employed to prepare thin films. In such reactors, film thickness and composition generally vary as a function of position within the reactor, and ion bombardment flux and energy to the growing film surface are not controlled. In addition, substrate temperature has not been controlled in this reactor configuration. Furthermore, no attempts were made to control crosslink density by varying the process conditions. Finally, scale-up of this type of volumetric reactor for manufacturing is expected to be difficult at best.

\subsection{Applications in sensors and actuators}

Hydrogels, mostly in the form of thin films, have found applications in sensors as the active layers on a variety of transducers. Certain hydrogel layers possess inherent sensitivity to analytes such as moisture and volatile organic compounds, and thus may be used in sensors. Sensing is accomplished by monitoring changes in the transducer signal in response to the analyte, which can adsorb and interact with the hydrogel matrix through various non-covalent forces ${ }^{78,79}$. However, such sensors suffer from poor selectivity due to cross or multiple reactivity. 
On the other hand, sensitivity for chemical and biological sensing may be imparted by incorporating various molecular recognition agents or catalysts in the hydrogel matrix, which can bind or react with analytes. The binding or catalytic event causes a change in the transducer signal, and hence allows sensing. Since molecular binding and catalysis are selective, sensors built on such principles are highly selective to specific analytes.

As discussed earlier, hydrogels are capable of swelling when exposed to water; the volume change in hydrogels has therefore led to applications in actuators. Smart hydrogels, which respond to various physical stimuli through volume changes, have found applications as self-powered actuators in micropumps and valves on microfluidic devices; recently hydrogel actuators that produce dimensional changes of $\sim 100 \%$ in less than 10 seconds as a result of $\mathrm{pH}$ change have been reported ${ }^{80}$. However, the thicknesses of hydrogel layers that can be used in microfluidic devices are generally in the micrometer range and higher, and will not be discussed further. The actuation response time of hydrogels is dictated by their size and water diffusivity since the volume changes are induced by diffusion of water molecules into and out of the hydrogel matrix. In general, since the actuation response time is directly proportional to the square of the characteristic dimension and inversely proportional to the diffusion coefficient, smaller dimension hydrogels have faster response times ${ }^{81}$.

Sub-micron thick hydrogel thin films may be expected to possess fast response times, and are also suitable for application in sensors. Stimuli responsive hydrogel films 
represent a sensor-actuator combination with great potential for novel analytical applications; the hydrogel films respond to physical, chemical or biological stimuli through volume changes, which may be quantified by transducers. Hydrogel films have been used to sense $\mathrm{pH}$, glucose, ions, and antigens. The transducers may be distinguished by the transduction principle used; the actuation response has been transduced into mechanical or mass, optical, thermal, and electrical or electrochemical signals. Some examples of environmentally sensitive hydrogels that serve as sensing elements, and transducers used to monitor actuation responses of the hydrogels reported in the literature, are given below.

$\mathrm{pH}$-sensitive hydrogels respond to changes in $\mathrm{pH}$ due to ionization of the acidic or basic sidegroups. By monitoring the volume changes of hydrogels in response to $\mathrm{pH}$, it is possible to sense $\mathrm{pH}$. Swelling induced by $\mathrm{pH}$ changes has been monitored through conductimetry $^{82}$, deflection of silicon membranes in piezoresistive pressure sensors ${ }^{83}$, changes in the holographic diffraction wavelength of holographs recorded in $\mathrm{pH}$-sensitive hydrogels ${ }^{84}$, shifts in the resonant frequency of quartz crystal microbalance ${ }^{85}$, and deflection of hydrogel coated microcantilevers ${ }^{85}$.

Glucose-sensitive hydrogels have been developed by incorporating glucose oxidase, or lectins such as concanavalin A, or phenylboronic acid moieties in the hydrogel matrix ${ }^{86}$. Glucose oxidase converts glucose to gluconic acid, thereby causing a decrease in the $\mathrm{pH}$ of the hydrogel microenvironment; if the hydrogel is $\mathrm{pH}$-sensitive, it will respond with a volume change. Although no sensors have been built to take 
advantage of this type of sensor-actuator mechanism, perhaps due to the popularity of amperometric determination of glucose, several self-regulating insulin delivery systems have been proposed ${ }^{86}$. Lectins such as concanavalin A (Con A) are carbohydrate binding proteins, and have also been used to develop glucose-sensitive hydrogels. Sensors based on the affinity binding of Con A to glucose have been demonstrated ${ }^{87}$, but they rely on monitoring the binding event itself and not on the binding induced swelling of a hydrogel film. Self-regulated insulin delivery systems based on Con A loaded hydrogels have been demonstrated ${ }^{88,89}$. Phenylboronic acid moieties are capable of binding glucose through ligand-receptor interactions, and have been incorporated into hydrogel matrices to develop glucose responsive hydrogels. Several holographic sensors for glucose have been demonstrated by conjugating phenylboronic acid moieties to hydrogel networks; upon binding with glucose, due to the change in osmotic pressure the hydrogel matrix experiences a volume change, which is quantified by a change in the diffraction wavelength of the hologram ${ }^{90,91}$. Further, when phenylboronic acid moieties are conjugated to a thermoresponsive polymer such as N-Isopropylacrylamide, binding with glucose modulates the LCST temperature of the hydrogel matrix; glucose sensing can then be accomplished by studying the changes in LCST temperatures of the hydrogel films $^{92}$

Divalent cations such as $\mathrm{Ca}^{2+}$ form ionic crosslinks with alginate hydrogels and hence, cause shrinking or swelling in response to environmental changes in the ion concentration. Optical sensors, which use diffraction gratings recorded in the alginate gels have been demonstrated for the detection of ions; the diffraction and reflection 
characteristics of the ion-sensitive hydrogel grating change in response to ion concentration $^{93}$. Sensors using other types of ion-sensitive hydrogels such as hydrogels impregnated with metal chelating monomers such as iminodiacetic acid $^{94}$, ligands such as nitriloacetic acid $^{95}$, crown ethers that complex metal ions ${ }^{96}$, and tetraalkylammonium salts that complex various anions ${ }^{97}$, have also been used to construct ion sensors. Detection of ions was accomplished by quantifying hydrogel volume changes as a result of the complexation event.

Antigen responsive hydrogels are a relatively new development, and appear to consist of reversible crosslinks formed between an antigen and the corresponding antibody immobilized in the hydrogel network ${ }^{98}$. When exposed to free antigen, the hydrogel swells because the free antigen displaces the immobilized antigen bound to the antibody in the network, resulting in the dissociation of physical crosslinks in the hydrogel. Recently, label-free biosensing of antigens has been demonstrated using hydrogel microlenses that contain reversible antigen-antibody crosslinks; upon exposure to free antigen, the focal length of the microlenses changed due to swelling caused by the dissociation of the physical crosslinks between bound antigen and antibody ${ }^{99}$. Antigen responsive hydrogels may also be developed using polymerizable antibody $\mathrm{F}_{\mathrm{ab}}{ }^{\prime}$ fragments ${ }^{100}$. The variable $\mathrm{F}_{\mathrm{ab}}$ ' segment of the antibody, which can be copolymerized in a hydrogel matrix, can bind antigens; osmotic pressure changes as a result of the binding event cause the hydrogel to swell or shrink. 
In addition to the chemical characteristics of the active layer, the process of preparing the thin film controls the performance and stability of a sensor. Traditional methods of forming polymer and hydrogel films such as spin coating from liquids, result in films that are soft, may not be homogeneous in thickness or properties, are difficult to form controllably and uniformly at thicknesses $<300 \mathrm{~nm}$, often display poor adhesion, require toxic, flammable solvents for spin-casting, and are frequently unstable over time ${ }^{101}$. Improvement in stability can be achieved through an additional fabrication step involving radiation exposure to crosslink the film, but device sensitivity may be compromised $^{102}$. Alternatively, hydrogel molecules can be chemically or physically grafted onto surfaces by the use of a coupling agent followed by solution polymerization ${ }^{103}$. This approach complicates the process and requires the development of specific coupling agents for different device surfaces, thereby inhibiting efficient manufacturing. In contrast, plasma polymerization can be used to produce pinhole free, spatially uniform, chemically, and physically stable polymer and hydrogel thin films for sensor devices ${ }^{104}$. Furthermore, plasma polymerization can be used to mass produce thin films for the production of sensor devices.

Early reports on the use of plasma polymerized films in sensors describe sensing layers that relied on inherent sensitivity of the plasma polymers to the analytes. Organosilicon polymers based on hexamethyldisiloxane and hexamethyldisilazane have been used to detect moisture and organic vapors by monitoring changes in their capacitive properties $^{105-107}$ or through the use of acoustic wave resonators ${ }^{101,108-111}$. Metal containing plasma polymers based on acetylacetonates ${ }^{112}$ and phthalocyanines ${ }^{113}$ have been used to 
sense reducing vapors such as $\mathrm{NO}_{2}$ and polycyclic compounds ${ }^{114}$ by monitoring changes in the film resistance and in the frequency of quartz crystal microbalances coated with the film. In addition, conductive polymers formed through plasma polymerization of pyrrole ${ }^{115}$ and thiophene ${ }^{116}$ have been used in chemiresistive sensors to detect organic vapors.

Recently, biosensors based on plasma polymers have been developed by conjugating molecular recognition agents such as antibodies and enzymes to the surface of transducers coated with plasma polymerized films. A surface plasmon resonance biosensor was constructed using plasma polymerized hexamethyldisiloxane film that was conjugated to bovine serum albumin and used to detect anti-BSA in solution ${ }^{117}$. Due to the relative ease of conjugating various biomolecules to amine terminated surfaces $^{118}$, plasma polymers with terminal, primary amine groups have been used to conjugate either antibodies or enzymes: amperometric sensors for glucose were fabricated by conjugating glucose oxidase to an amine terminated plasma polymer of acetonitrile through glutaraldehyde crosslinking procedure ${ }^{119-121}$; surface plasmon resonance and quartz crystal microbalance immunosensors were developed by attaching antibodies to amine terminated ethylenediamine plasma polymers through glutaraldehyde crosslinking ${ }^{122,123}$; ethylenediamine plasma polymers were also used in potentiometric acetylcholine chloride sensors by conjugating acetylcholinesterase to the terminal amine groups of the polymer by the glutaraldehyde crosslinking procedure ${ }^{124}$. In slightly different implementations, amine terminated surfaces of plasma polymerized n-butyl amine films were used to adsorb nanogold particles or an alginate polyelectrolyte layer, which then allow adsorption of antibodies or their fragments through chemical or electrostatic adsorption forces ${ }^{125-128}$; the 
assembly of antibodies on the plasma polymerized films could be used in a variety of immunosensing platforms. Since electrostatic adsorption forces may be modulated by changing the $\mathrm{pH}$, an immunosensor could be regenerated by desorbing the antibody supporting alginate polyelectrolyte layer from the amine terminated plasma polymer surface in strongly acidic or basic solutions ${ }^{125}$.

In this work, plasma polymerized NIPAAm films are used as the sensing layers in acoustoelectronic immunosensors based on surface acoustic wave (SAW) devices. Antibodies are incorporated into the plasma polymerized NIPAAm films using a novel, electrophoretic incorporation procedure, which provides orientation control of the antibodies. Further details on the procedure and sensor development may be found in Chapter 5. The key advantages of using plasma polymerized hydrogels in immunosensors include lower insertion loss of the acoustic devices relative to the use of conventionally processed hydrogel films, and the potential application of the technology for mass production of sensors. 


\section{CHAPTER 3}

\section{SYNTHESIS AND CHARACTERIZATION}

\subsection{Introduction}

Hydrogels are water-swollen crosslinked polymeric structures derived from hydrophilic monomers, and are produced by the polymerization of one or more monomers and involve interactions such as hydrogen bonding and strong van der Waals interactions between polymeric chains ${ }^{81}$. During plasma polymerization, crosslinks are built into the polymeric network without the need for crosslinking agents. Immediately following synthesis in the dry plasma state, the polymeric networks are glassy due to the absence of water and have properties similar to those of other glassy polymers; when the crosslinked polymer networks are exposed to water, they can absorb water up to several times their own weight, resulting in the formation of a hydrogel.

Crosslink density is an important parameter, and determines various properties of polymeric networks and hydrogels such as water uptake capacity, degree of hydrophilicity, and mechanical modulus ${ }^{129}$. In the case of intelligent hydrogels such as poly(N-Isopropylacrylamide), the degree of crosslinking dictates the parameters of the volume phase transition, such as the lower critical solution transition (LCST) temperature and the width of transition ${ }^{7}$. To obtain hydrogel films with the desired combination of properties, crosslink density may be varied through the addition of a crosslinking agent. However, the unreacted crosslinker needs to be leached out of the hydrogel network at 
the end of the crosslinking process. In contrast, during plasma polymerization, crosslinking is achieved without the use of a crosslinking agent. Furthermore, the degree of crosslinking in the hydrogel films can be tailored by varying process conditions such as substrate temperature, reactor operating pressure, and power coupled to the plasma.

Immobilizing thermoshrinking polymers in the form of thin films on a solid support such as a silicon wafer allows expansion and contraction essentially only in the direction normal to the substrate in response to temperature changes. The resulting temperature-sensitive hydrogel structure offers the potential for sensor and actuator applications ${ }^{81,130,131}$ and intelligent surfaces ${ }^{12}$ in biotechnology and medicine. Recently, the effects of lateral confinement of hydrogel films ${ }^{102,131}$ and possible applications have been studied. The majority of the investigations have centered on the preparation of hydrogel films using solution-based, free-radical polymerization or photopolymerization that necessitate the use of a crosslinker. Moreover, formation of thin films of hydrogels in this manner requires an additional step such as spin casting. In this chapter, a single step method of obtaining hydrogel thin films through plasma polymerization is described. So far, only a few studies have been reported on the use of plasma-assisted methods for hydrogel film formation. Details of these studies have been given in Chapter 2, and will not be repeated here.

NIPAAm films on silicon surfaces are prone to delamination and are not stable in aqueous environments, thus hindering film characterization and use in aqueous media. Adhesion limitations of NIPAAm films to silicon surfaces have been approached in various ways; anchoring NIPAAm chains to the surface using covalent linkages has been 
a common approach. Specifically, an adhesion promoter based on a monochlorosilane anchor group and a chromophore head group has been used to overcome poor adhesion of NIPAAm to silicon $^{132}$. Other silane based surface pretreatments have also been reported: $\gamma$-methacryloxypropyl trimethoxysilane ${ }^{18,133}, 3$-aminopropyl triethoxysilane ${ }^{134}$, vinyl triethoxysilane ${ }^{103}$, (N,N'-Diethylamino) dithiocarbamoyl propyl- (triethoxy) silane ${ }^{135}$. In addition, NIPAAm brushes have been grafted to hydroxyl terminated alkylthiolate monolayers on gold surfaces ${ }^{15}$. In an attempt to produce plasma polymerized NIPAAm thin films with improved adhesion, ${ }^{19}$ higher rf powers were invoked initially in the plasma reactor. Presumably the initial film layer thereby had higher carbon content than the remaining film, which was deposited under lower power conditions. No mention was made in these studies regarding whether NIPAAm film delamination occurs in conjunction with partial dissolution of the films, whether it is possible to improve the adhesion of the films, or whether it is possible to improve film adhesion by increasing the crosslink density during deposition. In this chapter, deposition and characterization of NIPAAm films, which display limited dissolution, excellent adhesion to silicon substrates, and thermoresponsive characteristics are described. The NIPAAm thin films were deposited in a parallel plate, plasma reactor directly on silicon substrates, without the use of an adhesion promoting layer.

\subsection{Experimental section}

Plasma polymerized NIPAAm thin films were deposited on silicon wafers in a capacitively coupled, $13.56 \mathrm{MHz}$ plasma reactor, details of which have been described

previously ${ }^{27}$. The electrodes in the reactor consisted of two stainless steel plates $(4 \mathrm{~cm}$ 
diameter) placed parallel to each other, and separated by 2" in all experiments. Power from an RF generator operating at $13.56 \mathrm{MHz}$ (ENI power systems: HF-300) was coupled to the top electrode through an antenna tuner (Heathkit SA 2060A), whereas the bottom electrode upon which the substrates were placed, and the body of the reactor were electrically grounded. A schematic of the reactor is shown in Figure 3.1. Prior to the deposition of NIPAAm films the substrate surface was activated by exposing it to an oxygen plasma for one minute at $133.3 \mathrm{~Pa}$ and $30 \mathrm{~W}$ rf power. The substrate was maintained at the same temperature used for subsequent deposition of NIPAAm films; four different temperatures of $120^{\circ} \mathrm{C}, 150{ }^{\circ} \mathrm{C}, 175{ }^{\circ} \mathrm{C}$ and $200{ }^{\circ} \mathrm{C}$ were used in this study. Prior to deposition, the surface pretreatment will likely create surface radicals, reduce organic contamination on the substrate surface, and thus enhance the adhesion of NIPAAm films to the substrate.

Since NIPAAm is a crystalline solid at ambient conditions, (melting point $=63$ ${ }^{\circ} \mathrm{C}$; boiling point $=85-88^{\circ} \mathrm{C}$ at $133.3 \mathrm{~Pa}$ ) it was vaporized by heating $10 \mathrm{~g}$ of the solid to $110^{\circ} \mathrm{C}$ in a glass storage flask. A threaded Teflon plug was used to regulate the introduction of NIPAAm vapor into gas delivery tubes. Stainless steel gas delivery tubes were maintained at $\sim 90{ }^{\circ} \mathrm{C}$ using heating tapes to avoid vapor condensation. Argon was used as the diluent in a 50:50 ratio to NIPAAm vapor in all experiments. NIPAAm thin films were deposited at platen temperatures of $120^{\circ} \mathrm{C}, 150^{\circ} \mathrm{C}, 175^{\circ} \mathrm{C}$ or $200{ }^{\circ} \mathrm{C}$, and pressures of 93.3 $\mathrm{Pa}$ or 133.3 $\mathrm{Pa}$. The resulting films were characterized with variable angle spectroscopic ellipsometry (VASE) (J. A. Woollam M-2000VI), contact angle measurements (FTA 32) and FTIR spectroscopy (Bruker IFS $66 \mathrm{v} / \mathrm{s}$ spectrometer). 
Ellipsometry was also used to determine the film thickness before and after water exposure.

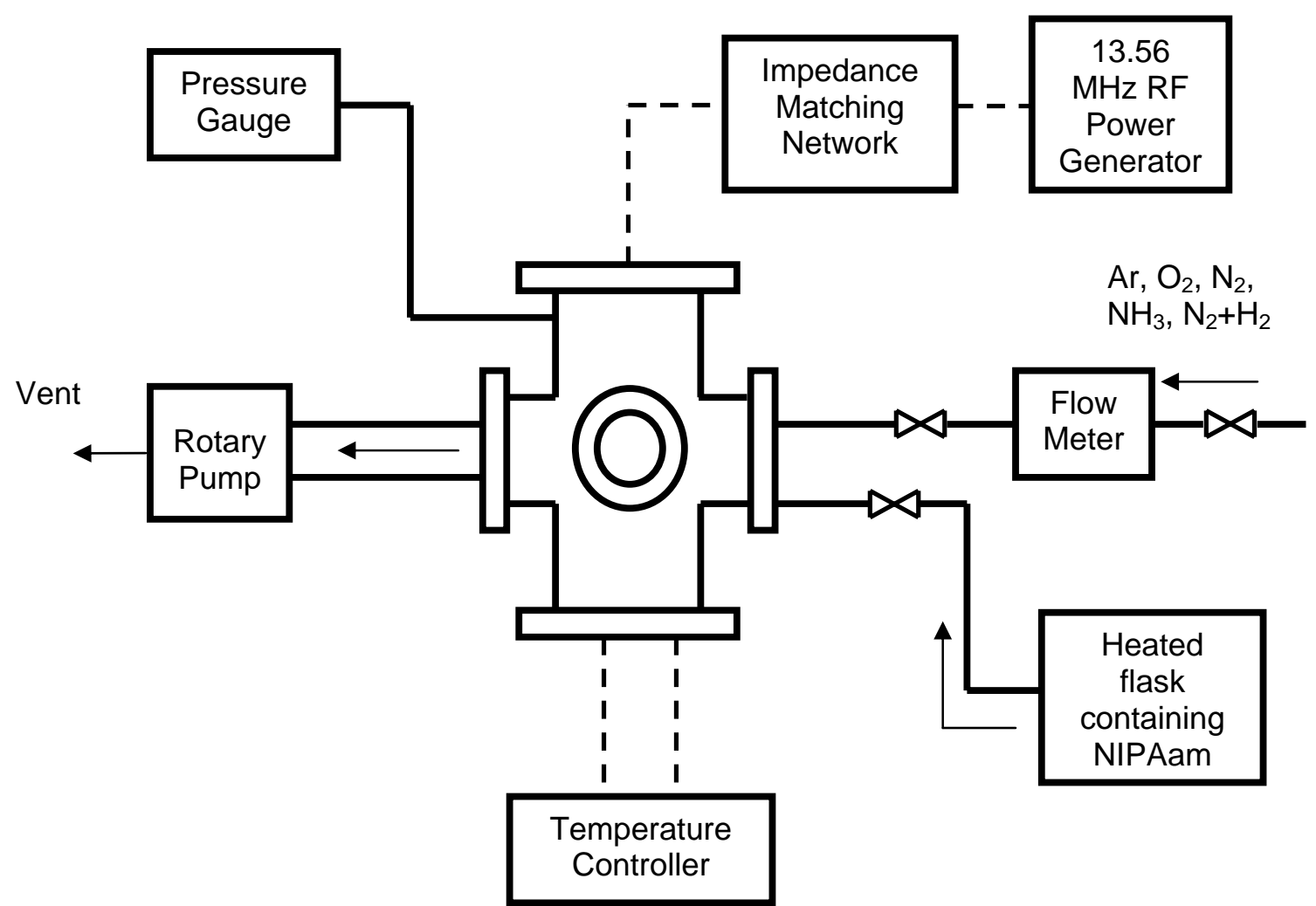

Figure 3.1 Schematic of the capacitively coupled, parallel plate plasma reactor used to deposit NIPAAm thin films

With the help of contact angle measurements, it was possible to determine the relative hydrophilicity / hydrophobicity of the NIPAAm surfaces. FTIR spectroscopy permitted the determination of chemical bonding information before exposure to water.

Thickness of the NIPAAm films was determined by fitting experimental ellipsometry data ( $\delta$ and $\psi$ ) obtained at three different angles of $65^{\circ}, 70^{\circ}$, and $75^{\circ}$, and at 
different wavelengths ranging from $400-1000 \mathrm{~nm}$ to Cauchy layers. The dispersion relation of a Cauchy layer is given by

$$
n=A+\frac{B}{\lambda^{2}}+\frac{C}{\lambda^{4}}
$$

where, $n$ is the refractive index of the layer, $\lambda$ is the wavelength, and $A, B$, and $C$ are Cauchy constants. Fitting was performed through a Marquardt-Levenburg algorithm in the WVASE32 software provided by J A Woollam Co., Inc. so that the mean square error (MSE) associated with the fit is less than 30. Furthermore, fits to the data were discarded if the refractive index of fitted Cauchy layer did not show a monoton decrease with increase in the wavelength.

Contact angle measurements were performed using 18.0 M $\Omega$-cm deionized water to determine the surface hydrophilicity / hydrophobicity of the NIPAAm films. Prior to contact angle measurements, NIPAAm samples were rinsed in deionized water three times to remove uncrosslinked monomer chains ${ }^{13}$; excess water was removed by blowdrying using filtered compressed air. This procedure is also believed to hydrate the polymer, which was crosslinked in the "dry", plasma state. Contact angle measurements were performed on a custom built thermoelectric heating/cooling stage; temperature was controlled to $\pm 0.2{ }^{\circ} \mathrm{C}$ using a board level Proportional-Integral-Derivative temperature controller (Oven Industries 5C7-550). The temperature controller used a thermistor to sense the temperature of the thermoelectric stage; independent confirmation of the temperature was obtained using a thermocouple. Although the thermocouple and thermistor probes were placed near the sample surface to minimize errors in measurement of the temperature of the sample surface, spatial temperature variations of up to $2{ }^{\circ} \mathrm{C}$ were 
found on the thermoelectric stage. The thermocouple temperature readings had a linear correlation with the thermistor temperatures $\left(\mathrm{R}^{2}=0.998\right)$, which allowed a simple calibration.

Contact angles were determined from spherical fits of images of $10 \mu \mathrm{l}$ deionized water drops on the surface of NIPAAm films. Images of the sessile drops on the sample surface were recorded using a camera, which could be triggered immediately before the drop contacted the surface. By adjusting parameters on the software program used to control the syringe containing the deionized water and the camera, an image was captured each second for $120 \mathrm{~s}$. In all cases, measured contact angles decrease from the time of initial contact of the water droplet with the hydrogel surface. The contact angles reported in the following discussion were recorded $60 \mathrm{~s}$ after the drop contacted the surface in most cases; at this point, the contact angle no longer changed with time. When the sample temperature was greater than $32^{\circ} \mathrm{C}$, the droplet volume decreased drastically $10 \mathrm{~s}$ after the drop contacted the surface due to water evaporation. In order to ensure that the contact angles measured were those of sessile drops and not receding drops, the contact angles were recorded $10 \mathrm{~s}$ after the water drop contacted the surface. The contact angles reported are the average of at least three measurements at each temperature; all contact angles were recorded at a relative humidity value of approximately $40 \%$.

FTIR spectra of NIPAAm thin films deposited on silicon wafers were collected in the transmission mode at a resolution of $4 \mathrm{~cm}^{-1}$. All spectra were averaged over 256 scans to minimize the noise and maximize the signal-to-noise ratio, and ratioed to the 
spectrum from a blank silicon wafer to account for the background. Furthermore, to prevent interference from water in the environment, the samples were subjected to a vacuum (200 Pa) while the spectra were being recorded.

\subsection{Results and Discussion}

Film thickness before and after exposure to water was determined using VASE and model fitting. Deposition rates in $\mathrm{nm} / \mathrm{min}$ were calculated from the film thickness and deposition time; results are presented in Table 3.1. Under all deposition conditions, a change in film thickness was observed after water exposure. Negative relative changes in thickness are caused by partial film dissolution or adhesion loss, while positive relative changes in thickness are due to film swelling. Lack of adequate crosslinking can cause dissolution of the films, since uncrosslinked hydrophilic chains of the polymer dissolve in water. Higher substrate temperatures and lower deposition pressures (higher ion bombardment energy) are expected to lead to more highly crosslinked films, which may not show dissolution or adhesion loss. Lower substrate temperatures and higher deposition pressures should result in less crosslinked films that are prone to film dissolution. Thus, an optimum set of deposition conditions is expected; indeed results presented in Table 3.1 are consistent with this hypothesis. Films deposited at $200{ }^{\circ} \mathrm{C}$ and 93.3 $\mathrm{Pa}$ show the highest positive relative change in thickness, while films deposited at $120^{\circ} \mathrm{C}$ and $133.3 \mathrm{~Pa}$ display the highest negative relative change in thickness. 
Table 3.1 VASE results of NIPAAm films deposited on silicon

\begin{tabular}{|c|c|c|c|c|c|}
\hline $\begin{array}{c}\text { Substrate } \\
\text { temperature } \\
\left({ }^{\circ} \mathrm{C}\right)\end{array}$ & $\begin{array}{c}\text { Deposition } \\
\text { pressure } \\
(\mathrm{Pa})\end{array}$ & $\begin{array}{c}\text { Deposition } \\
\text { rate } \\
(\mathrm{nm} / \mathrm{min})\end{array}$ & $\begin{array}{c}\text { Thickness } \\
\text { before } \\
\text { exposure to } \\
\text { water }(\mathrm{nm})\end{array}$ & $\begin{array}{c}\text { Thickness } \\
\text { after } \\
\text { exposure } \\
\text { to water } \\
(\mathrm{nm})\end{array}$ & $\begin{array}{c}\% \text { Change } \\
\text { in } \\
\text { thickness }\end{array}$ \\
\hline \multirow{2}{*}{120} & 133.3 & 56.3 & 284.0 & 39.1 & -86.2 \\
\cline { 2 - 6 } & 93.3 & 22.0 & 321.7 & 151.4 & -52.9 \\
\hline \multirow{2}{*}{150} & 133.3 & 10.8 & 130.6 & 135.7 & 3.9 \\
\cline { 2 - 6 } & 93.3 & 12.7 & 117.7 & 111.2 & -5.5 \\
\hline \multirow{2}{*}{175} & 133.3 & 17.1 & 342.2 & 342.0 & -0.1 \\
\cline { 2 - 6 } & 93.3 & 3.6 & 55.2 & 52.0 & -5.7 \\
\hline \multirow{2}{*}{200} & 133.3 & 2.7 & 55.5 & 61.6 & 11.0 \\
\cline { 2 - 6 } & 93.3 & 2.0 & 22.5 & 28.4 & 25.9 \\
\hline
\end{tabular}

The above results are in agreement with previous studies that used substrate temperature variation to control film chemistry in the plasma polymerization of 2hydroxyethyl methacrylate, hexafluorobutadiene, ethylene oxide and tetrahydrofuran ${ }^{26}$. Lower substrate temperatures yield films with chemical composition identical to that of the monomer. At higher substrate temperatures, the increased thermal energy of reactive species can result in greater bond scission and chemical reactivity. In the current study, lower pressure results in higher ion bombardment energy of the growing film surface and thus enhanced crosslinking.

Film deposition rate, which is a function of reactor conditions, is indicative of film properties. Indeed, our results demonstrate that at $120^{\circ} \mathrm{C}$ and $133.3 \mathrm{~Pa}$, NIPAAm 
films were deposited at a rate of $56.3 \mathrm{~nm} / \mathrm{min}$, which is the highest rate observed under the conditions investigated; as indicated previously, these films showed the greatest reduction in thickness after water exposure. In comparison, films deposited at $200{ }^{\circ} \mathrm{C}$ and $93.3 \mathrm{~Pa}$, displayed the lowest deposition rate of $2.0 \mathrm{~nm} / \mathrm{min}$ and showed the highest positive relative change in thickness upon water exposure.

\subsubsection{Infrared Spectroscopy Characterization of Hydrogel Films}

FTIR spectra of plasma polymerized NIPAAm films are presented in Figs. 3.2 \&

3.3. Chemical composition of the plasma polymerized NIPAAm thin films were studied using FTIR; wavenumbers of the primary absorption bands and the bonding structures of various samples are provided in Table 3.2. The amide I $\left(\sim 1640-1680 \mathrm{~cm}^{-1}\right)$ and amide II $\left(\sim 1520-1540 \mathrm{~cm}^{-1}\right)$ bands associated with $\mathrm{C}=\mathrm{O}$ stretching and $\mathrm{N}-\mathrm{H}$ stretching of secondary amides respectively, are critical to understanding the structure of NIPAAm. These bands are prominent in FTIR spectra of NIPAAm, and are sensitive to the degree

and type of hydrogen bonding ${ }^{136,137}$. Deconvolution and second derivative spectroscopy have revealed various subbands that are shifted to lower wavenumbers as a result of hydrogen bonding with the amide I structure: "free", non-hydrogen bonded $\mathrm{C}=\mathrm{O}$ 


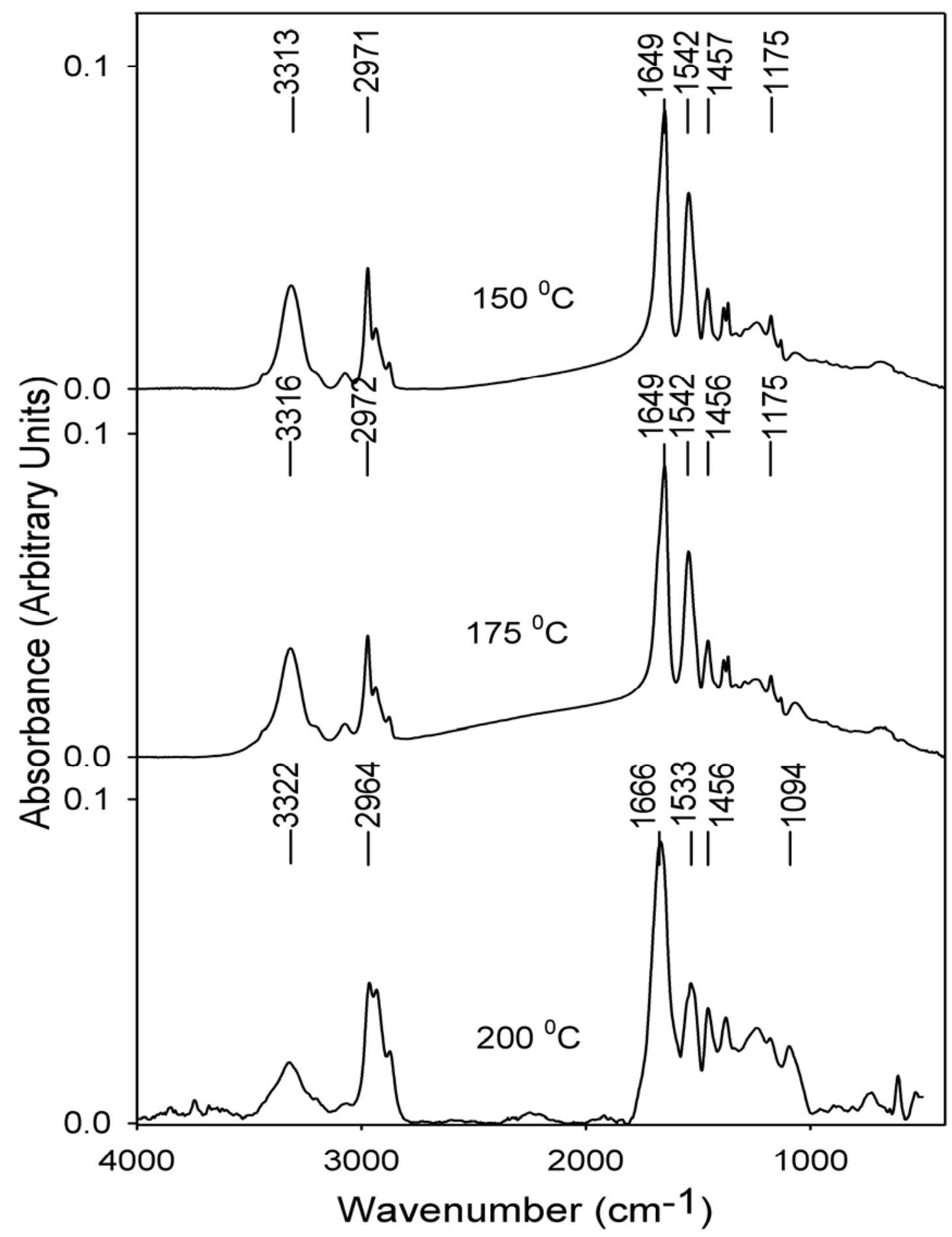

Figure 3.2 FTIR spectra of NIPAAm films deposited on silicon at $133.3 \mathrm{~Pa}$ at $150{ }^{\circ} \mathrm{C}$, $175^{\circ} \mathrm{C}$ and $200{ }^{\circ} \mathrm{C} ; 30 \mathrm{~W}$ rf power. 


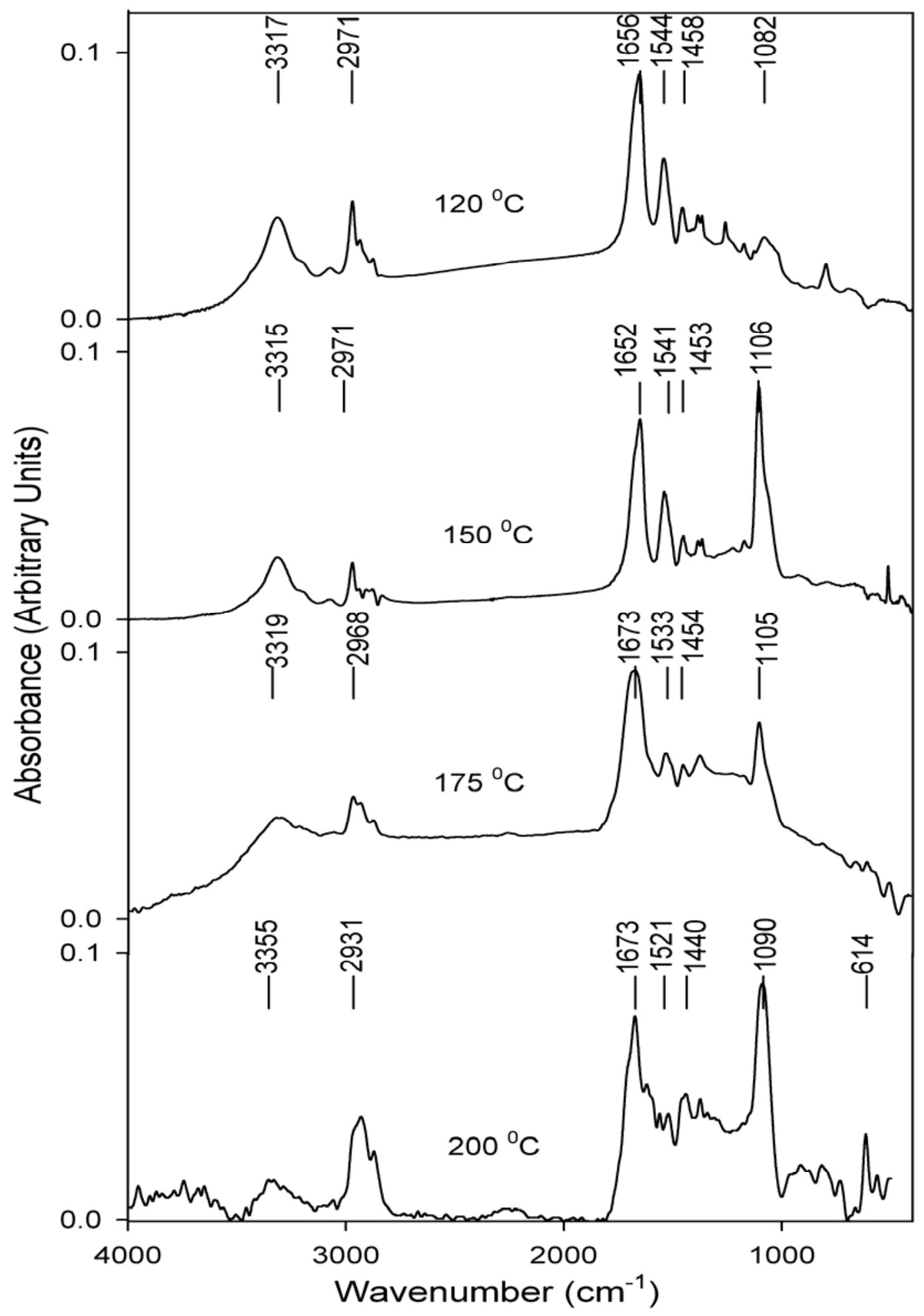

Figure 3.3 FTIR spectra of NIPAAm films deposited on silicon at $93.3 \mathrm{~Pa}$ at $120{ }^{\circ} \mathrm{C}, 150$ ${ }^{\circ} \mathrm{C}, 175^{\circ} \mathrm{C}$ and $200{ }^{\circ} \mathrm{C} ; 30 \mathrm{~W}$ rf power. 
Table 3.2. FTIR band assignments for NIPAAm films deposited on silicon

\begin{tabular}{|c|c|c|c|c|c|c|c|}
\hline $\begin{array}{c}200^{\circ} \mathrm{C} \\
93.3 \mathrm{~Pa} \\
\left(\mathrm{~cm}^{-1}\right)\end{array}$ & $\begin{array}{l}175^{\circ} \mathrm{C} \\
93.3 \mathrm{~Pa} \\
\left(\mathrm{~cm}^{-1}\right)\end{array}$ & $\begin{array}{c}150^{\circ} \mathrm{C} \\
93.3 \mathrm{~Pa} \\
\left(\mathrm{~cm}^{-1}\right)\end{array}$ & $\begin{array}{c}120^{\circ} \mathrm{C} \\
93.3 \mathrm{~Pa} \\
\left(\mathrm{~cm}^{-1}\right)\end{array}$ & $\begin{array}{c}200^{\circ} \mathrm{C} \\
133.3 \\
\mathrm{~Pa} \\
\left(\mathrm{~cm}^{-1}\right)\end{array}$ & $\begin{array}{c}175^{\circ} \mathrm{C} ; \\
133.3 \\
\mathrm{~Pa}\left(\mathrm{~cm}^{-}\right. \\
\left.{ }_{1}\right)\end{array}$ & $\begin{array}{c}150^{\circ} \mathrm{C} ; \\
133.3 \\
\mathrm{~Pa}\left(\mathrm{~cm}^{-}\right. \\
\left.{ }^{1}\right)\end{array}$ & Assignment \\
\hline \multirow[t]{3}{*}{1089.65} & 1105.03 & 1106.27 & 1081.87 & 1094.46 & 1067.87 & 1066.80 & $\begin{array}{l}\text { Asymmetric } \\
\text { Si-O-C } \\
\text { stretching }\end{array}$ \\
\hline & & 1173.82 & 1174.88 & & 1175.42 & 1175.32 & $\begin{array}{c}\text { Skeletal } \\
\text { vibration of - } \\
\mathrm{C}\left(\mathrm{CH}_{3}\right)_{2}\end{array}$ \\
\hline & & & 1260.44 & 1237.30 & 1239.75 & 1238.73 & Amide III band \\
\hline \multirow[t]{2}{*}{1374.38} & 1377.78 & 1366.89 & 1367.34 & 1376.22 & 1366.39 & 1366.65 & \multirow{2}{*}{$\begin{array}{l}\text { Antisymmetric } \\
\text { deformation of } \\
-\mathrm{C}\left(\mathrm{CH}_{3}\right)_{2}\end{array}$} \\
\hline & & 1385.41 & 1386.41 & & 1386.10 & 1386.36 & \\
\hline 1439.96 & 1454.46 & 1452.93 & 1457.75 & 1455.76 & 1455.91 & 1457.28 & $\begin{array}{c}\text { Symmetric } \\
\text { deformation of } \\
-\mathrm{C}\left(\mathrm{CH}_{3}\right)_{2}\end{array}$ \\
\hline 1520.79 & 1533.47 & 1541.31 & 1543.51 & 1533.04 & 1542.23 & 1541.99 & Amide II band \\
\hline 1673.40 & 1673.14 & 1652.13 & 1655.71 & 1666.19 & 1649.21 & 1648.91 & Amide I band \\
\hline \multirow[t]{2}{*}{2254.36} & 2261.05 & & & 2248.86 & & & \\
\hline & & & 2937.59 & 2932.69 & 2936.32 & 2935.45 & $\begin{array}{c}\text { Asymmetric } \\
\text { stretching of - } \\
\mathrm{CH}_{2}\end{array}$ \\
\hline 2930.56 & 2967.66 & 2971.49 & 2971.17 & 2964.02 & 2971.56 & 2971.25 & $\begin{array}{c}\text { Asymmetric } \\
\text { stretching of - } \\
\mathrm{CH}_{3}\end{array}$ \\
\hline 3354.99 & 3319.39 & 3315.10 & 3316.60 & 3322.35 & 3316.17 & 3312.89 & $\begin{array}{l}\mathrm{N}-\mathrm{H} \text { stretch of } \\
2^{\circ} \text { amide }\end{array}$ \\
\hline
\end{tabular}

stretching is observed at $\sim 1670 \mathrm{~cm}^{-1}$; weak intramolecular hydrogen bonded $\mathrm{C}=\mathrm{O}$ stretches at $\sim 1655 \mathrm{~cm}^{-1}$; strong intermolecular hydrogen bonded $\mathrm{C}=\mathrm{O}$ stretching is 
observed at $\sim 1629 \mathrm{~cm}^{-1} 138$. The opposite trend is found in the case of amide II bands: hydrogen bonding causes the amide II band to shift to higher wavenumbers. The nonhydrogen bonded, "free" band is found at $\sim 1535 \mathrm{~cm}^{-1}$, the intramolecular hydrogen bonded band at $\sim 1551 \mathrm{~cm}^{-1}$ and the intermolecular hydrogen bonded band at $\sim 1565 \mathrm{~cm}^{-1}$ 139. Analysis of the FTIR spectra of NIPAAm in Figs. $3.2 \& 3.3$ based on the trends described above shows that deposition at higher temperatures results in NIPAAm films with weak or no hydrogen bonding. This observation is significant from the standpoint of understanding the degree of hydration of NIPAAm films as a result of exposure to the ambient. Films deposited at lower temperatures $\left(120^{\circ} \mathrm{C}\right.$ and $\left.150^{\circ} \mathrm{C}\right)$ are more likely to be hydrated by water vapor in the laboratory environment than films deposited at higher temperatures $\left(175^{\circ} \mathrm{C}\right.$ and $\left.200{ }^{\circ} \mathrm{C}\right)$.

Existence of bands at $1172,1366,1386$ and $1455 \mathrm{~cm}^{-1}$ is considered proof that the isopropyl group of NIPAAm is preserved and not degraded due to exposure to the plasma environment. In the present study, two bands at $1365 \mathrm{~cm}^{-1}$ and $1386 \mathrm{~cm}^{-1}$, which are associated with antisymmetric deformation of the isopropyl group, were observed in all samples deposited at lower temperatures whereas a single band at $\sim 1377 \mathrm{~cm}^{-1}$, perhaps resulting from merging of the bands at 1365 and $1386 \mathrm{~cm}^{-1}$, was noted in NIPAAm deposited at higher temperatures. Furthermore, the band at $\sim 1172 \mathrm{~cm}^{-1}$, associated with skeletal vibration of $-\mathrm{C}\left(\mathrm{CH}_{3}\right)_{2}{ }^{140}$ is not observed in NIPAAm films deposited at higher temperatures. These changes, which signify a general loss of vibrational freedom, are likely associated with the greater crosslinking and conformational order that can be expected in NIPAAm films deposited at higher temperatures. As proof of this trend, the 
bands at $\sim 1451 \mathrm{~cm}^{-1}$ (symmetric deformation of $\left.-\mathrm{C}\left(\mathrm{CH}_{3}\right)_{2}\right)$ and $2969 \mathrm{~cm}^{-1}$ (asymmetric stretching of $-\mathrm{CH}_{3}$ ) shift toward lower frequencies with increasing deposition temperatures ${ }^{138}$. Furthermore, NIPAAm films deposited at higher substrate temperatures and lower pressures do not show the band at $\sim 2932 \mathrm{~cm}^{-1}$, which is assigned to the asymmetric stretch of the methylene group found in the backbone of NIPAAm. Absence of this band and hence, of the methylene group indicates that at higher substrate temperatures and lower reactor pressure, chain scission may occur.

The absorption band at $\sim 1080 \mathrm{~cm}^{-1}$, assigned to asymmetric stretching of the SiO-C bond, has differing widths and is prominent in films deposited at $93.3 \mathrm{~Pa}$ only. This suggests the existence of a covalent linkage between the silicon substrate and NIPAAm formed at 93.3 Pa. Although this band is present in NIPAAm films formed at $133.3 \mathrm{~Pa}$, it is weak in comparison to the other prominent bands in the spectra. Such observations are consistent with the reduced ion energy at higher pressures, which results in less bond breaking at the substrate surface as film deposition begins. Evidence of other types of covalent bonding between silicon and NIPAAm films formed at $133.3 \mathrm{~Pa}$ is not discernible. The absorption band due to $\mathrm{N}-\mathrm{H}$ stretching in secondary amides is found at $\sim 3315 \mathrm{~cm}^{-1}$ in all samples. The amide III band $\left(\sim 1230 \mathrm{~cm}^{-1}\right)$, which contains contributions from $\mathrm{N}-\mathrm{H}$ in plane bending and $\mathrm{C}-\mathrm{N}$ stretching, is present in all samples deposited at 133.3 Pa and in NIPAAm formed at $120{ }^{\circ} \mathrm{C}$ and $93.3 \mathrm{~Pa}$. The broad band observed at $\sim 2250 \mathrm{~cm}^{-1}$ in NIPAAm deposited at $200{ }^{\circ} \mathrm{C}$ and $175{ }^{\circ} \mathrm{C}(93.3 \mathrm{~Pa}$ only) has not been assigned to any specific chemical moiety since it is believed to be a combination peak arising from $\mathrm{HOH}$ bending modes ${ }^{139}$. 


\subsubsection{Thermoresponsive Properties of Hydrogel Films}

The thermoresponsive behavior of plasma polymerized NIPAAm films deposited under four different reactor conditions was investigated using contact angle measurements. Relatively high deposition rates and low net dissolution of the films were important criteria for the choice of samples studied. The dependence of contact angle on sample temperature is shown in Figure 3.4 (a-d). Error bars on the contact angle values represent standard deviations from the measurement averages. In all cases, nearly reversible thermoresponsive behavior is displayed. The arrows on the figures indicate the heating and cooling cycles. While the data points obtained on the heating and cooling cycles are within experimental error, the contact angles measured on the cooling cycle were almost always higher than those on the heating cycle. This is not surprising because in all cases data was obtained by beginning with the heating cycle. Hence, the contact angles measured on the cooling cycle were obtained after several measurements were made on the heating cycle during which, the sample surfaces were exposed to various organic contaminants in the laboratory environment and from the lint-free tissue used to absorb the droplets of water on the surface. The reason for deviation from this trend in the case of NIPAAm films deposited at $150{ }^{\circ} \mathrm{C}$ and $133.3 \mathrm{~Pa}$ (Figure 3.4 (b)) is not clear; it is most likely that a systematic error is introduced in the measurements. 

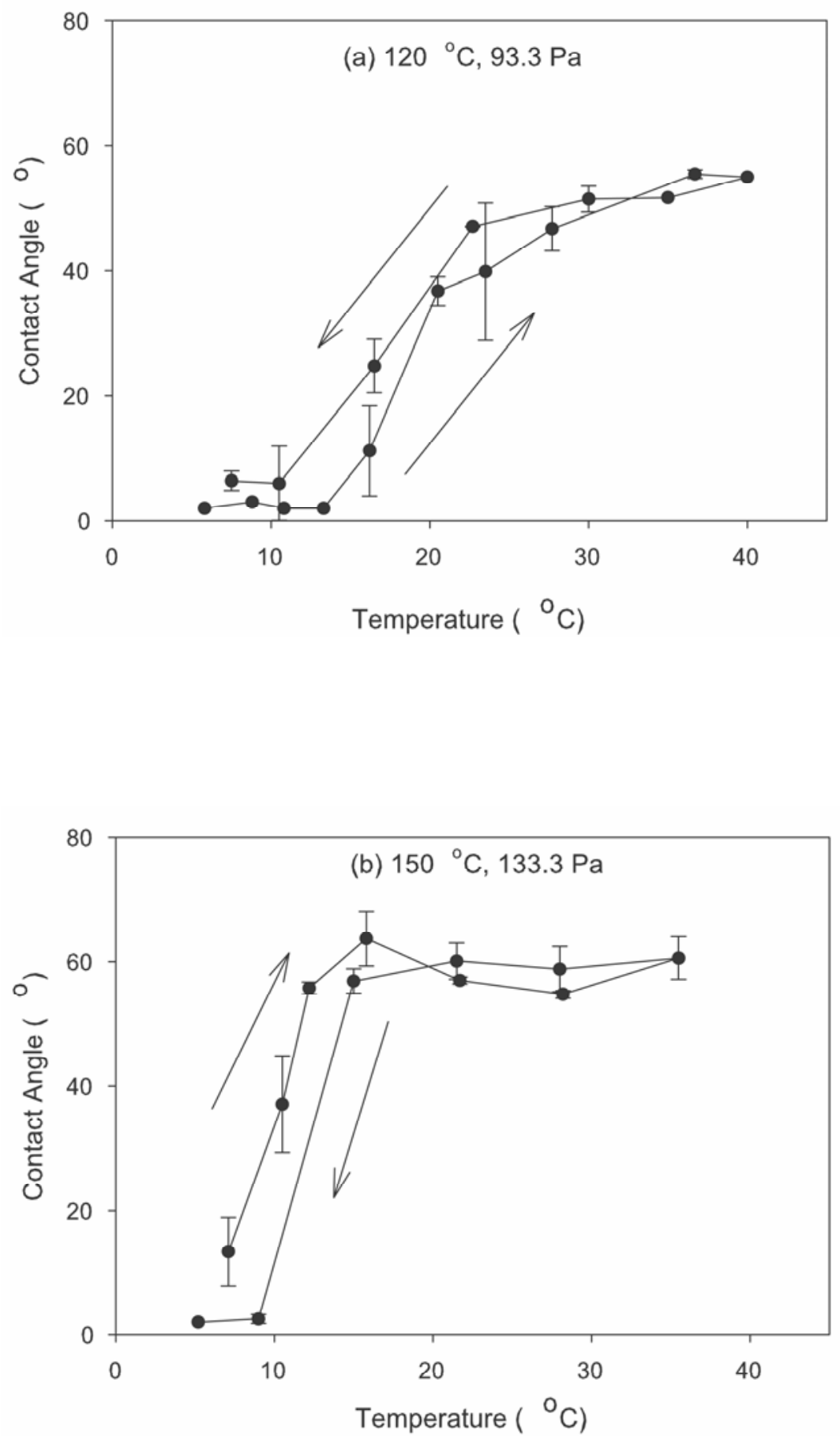

Figure 3.4 Contact angles of plasma polymerized NIPAAm on silicon surface deposited at (a) $120^{\circ} \mathrm{C}, 93.3 \mathrm{~Pa}$, (b) $150{ }^{\circ} \mathrm{C}, 133.3 \mathrm{~Pa}$. RF power was $30 \mathrm{~W}$ for all samples (continued on next page) 

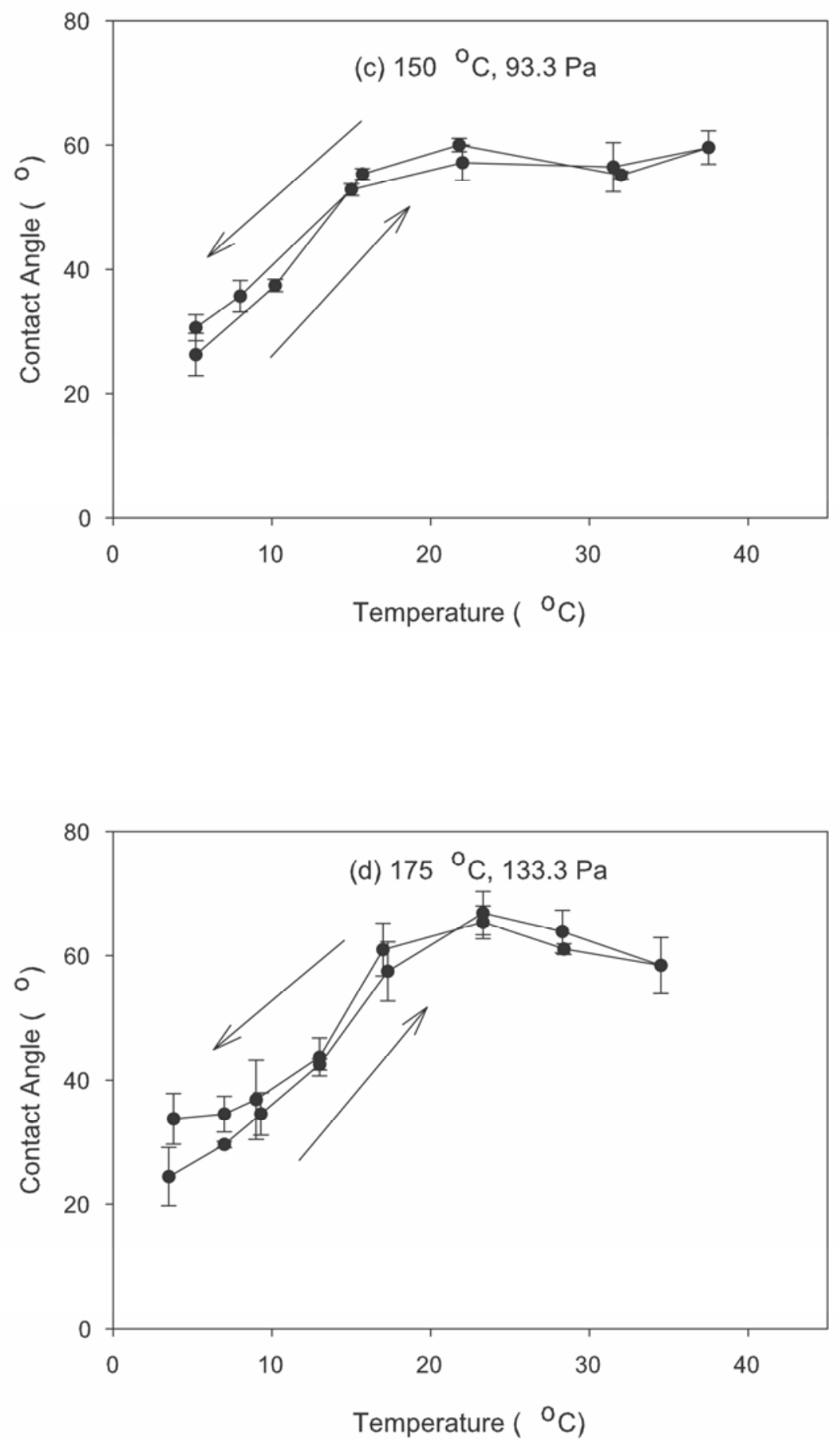

Figure 3.4 continued: Contact angles of plasma polymerized NIPAAm on silicon surface deposited at (c) $150^{\circ} \mathrm{C}, 93.3 \mathrm{~Pa}$, and (d) $175^{\circ} \mathrm{C}, 133.3 \mathrm{~Pa}$. RF power was $30 \mathrm{~W}$ for all samples. 
From Figure 3.4 it is clear that the plasma polymerized hydrogel thin films undergo a temperature-induced phase transition, wherein the affinity of the surface to water changes. At lower temperatures, the surface of plasma polymerized hydrogel films is hydrophilic, but at higher temperatures, the surface is less hydrophilic and hence, relatively hydrophobic. In all the cases studied, the difference in the water droplet contact angle as a result of the phase transition is at least $35^{\circ}$.

The hydrophilic-hydrophobic transition temperatures for the various samples were determined by numerically differentiating the data and computing the inflection point ${ }^{76}$. In all cases the data of both the heating and cooling cycles were used to determine the inflection point. The transition temperatures $\left(T_{c}\right)$ of the various samples were assigned by averaging the inflection points of the heating and cooling cycles and are as follows: $120^{\circ} \mathrm{C}, 93.3$ Pa: $18.2{ }^{\circ} \mathrm{C} ; 150{ }^{\circ} \mathrm{C} 133.3$ Pa: $12{ }^{\circ} \mathrm{C} ; 175^{\circ} \mathrm{C}, 133.3$ Pa: $13.7^{\circ} \mathrm{C} ; 150{ }^{\circ} \mathrm{C}, 93.3$

Pa: $9{ }^{\circ} \mathrm{C}$. The values of $\mathrm{T}_{\mathrm{c}}$ are significantly lower than those reported in the literature for NIPAAm $\left(31^{\circ} \mathrm{C}\right)^{7}$; these differences may be explained on the basis of the hydrophobic nature of plasma polymerized NIPAAm. It is well known that increasing the hydrophobic content of a hydrogel, for example by copolymerizing NIPAAm with a hydrophobic monomer, lowers the transition temperature ${ }^{7}$. Indeed transition temperatures as low as $24.7^{\circ} \mathrm{C}$ have been reported for NIPAAm co- and terpolymers based on the degree of hydrophobicity introduced into the hydrogel network through the chromophore used for the polymerization reaction ${ }^{102}$.

In a related study ${ }^{141}$, surfaces of end-grafted and multi-point grafted poly(NIPAAm) and its copolymers showed the hydrophilic-hydrophobic transition when 
dynamic contact angles of water were recorded as a function of temperature; terminally grafted surfaces were found to have a transition temperature at $24^{\circ} \mathrm{C}$. Furthermore, these studies demonstrated that the multi-point grafted surface display smaller changes in contact angles and hence, hydrophilic properties, than the end-grafted surface, possibly due to restrictions on the polymer chain mobility in the multi-point grafted surface. Such results are consistent with our observations since plasma deposition yields many surface attachment points for the hydrogel films due to ion/electron bombardment of the surface during deposition.

Based on FTIR studies, increased hydrophobic character and a partial loss of polar groups in the plasma polymerized hydrogel network are supported by the lack of significant hydrogen bonding in films deposited at higher temperatures and lower pressures. Furthermore, films deposited at higher temperatures and lower pressures exhibit chain scission as evidenced by the absence of the FTIR band at $2932 \mathrm{~cm}^{-1}$. The transition temperatures obtained for the four samples examined using contact angle goniometry support this trend: samples prepared at lower pressures and higher temperatures show lower $T_{c}$ than films deposited at lower temperatures and higher reactor pressures. Furthermore, the transition of plasma polymerized hydrogel thin films may be expected to be lower than the reported temperature for bulk poly (NIPAAm) since it was polymerized in the "dry" state. It is known that polymerization and crosslinking in the dry state induces a compressive stress on the hydrogel network when it is swollen, which contributes to a lowering of the transition temperature ${ }^{140}$. 
In addition to the trend in transition temperatures, the width of the transition merits attention. In the samples prepared at $120^{\circ} \mathrm{C}, 93.3 \mathrm{~Pa}$ and $150{ }^{\circ} \mathrm{C}, 133.3 \mathrm{~Pa}$, the transition is relatively sharp, whereas the transition is nearly continuous in the case of the other two samples. Short chains and inhomogeneous networks with a broad distribution of polymer chain lengths between crosslinks yield a continuous transition ${ }^{142}$. Thus, it is plausible that a broad transition occurs in films plasma deposited at lower pressures and higher temperatures; that is deposition under more energetic conditions results in extensive chain scission and crosslinking. Therefore, Figure 3.4 indicates that plasma polymerized hydrogel thin films of NIPAAm exhibit a reversible LCST phase transition since the plasma polymerization conditions allow retention of both hydrophilic and hydrophobic molecular groups that are necessary for the phase transition.

\subsection{Conclusions}

Thin films of NIPAAm were deposited in a parallel plate capacitively coupled rf plasma reactor. Reactor pressure and temperature were varied in order to alter film chemical and physical properties. Films deposited under lower temperature, higher pressure conditions displayed enhanced dissolution in water leading to significant loss in film thickness. NIPAAm films deposited at higher substrate temperatures and lower deposition pressures, were more stable in aqueous environments and showed little or no dissolution; films deposited under appropriate deposition conditions swelled when exposed to water. FTIR spectra of films deposited under different reactor conditions yielded insight into the resulting chemical structures. Contact angle measurements 
demonstrated that plasma polymerized NIPAAm films are capable of exhibiting a reversible LCST transition. By varying the reactor conditions, and hence crosslink density of the films, it is possible to tailor the transition temperature.

These studies represent the first step in producing plasma polymerized hydrogel thin films of NIPAAm in a capacitively coupled reactor as well as delineating and understanding the properties of the films formed under various reactor conditions. Good adhesion of the films to silicon surfaces was successfully achieved by polymerizing NIPAAm under conditions that lead to greater crosslinking and chain scission. Different volume phase transition behavior was observed as a result of minor changes in the chemical composition of the hydrogel films produced. These results are significant in that they demonstrate the potential of plasma polymerization to produce hydrogel thin films with excellent adhesion to the silicon surface without an intermediate adhesion promotion layer and indicate the feasibility of producing hydrogel films with welldefined phase transition behavior. 


\section{CHAPTER 4}

\section{WATER AND MOISTURE UPTAKE CHARACTERISTICS}

\subsection{Introduction}

Hydrogels are three-dimensional polymer networks that swell in aqueous solutions while maintaining their integrity. "Intelligent" hydrogels respond to a variety

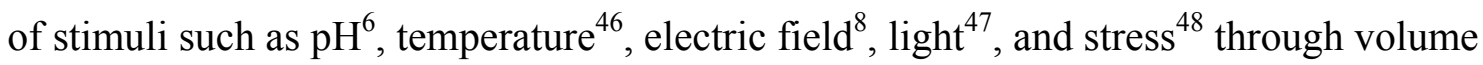
or conformation changes. The macroscopic changes are a result of fast, reversible changes at the microscopic level and have led to novel applications in drug delivery ${ }^{9,10}$, bioseparations $^{11}$, sensors ${ }^{1}$, and intelligent surfaces ${ }^{12}$. Poly (N-Isopropylacrylamide) (NIPAAm), a widely studied thermoresponsive polymer, exhibits inverse temperature solubility in aqueous solutions as a result of a volume phase transition analogous to lower critical solution transition (LCST) temperature in single linear chains. While a variety of techniques are available to study the LCST transition in bulk hydrogels, the number of analytical methods to characterize the thermoresponsive properties of thin films is limited. Furthermore, the presence of a constraining substrate in thin films makes interpretation of the results difficult. Nevertheless, several investigators have studied the LCST type of transition in NIPAAm thin films using optical techniques such as surface plasmon resonance ${ }^{15,18,131}$, and ellipsometry ${ }^{143}$, surface analytical techniques such as static $^{144}$ and dynamic contact angle ${ }^{145} 141135$ measurements, and atomic force microscopy ${ }^{19}$. 
In addition to the analytical techniques mentioned above, temperature dependent quartz crystal microbalance (QCM) measurements may be used to study mass changes associated with NIPAAm thin films upon exposure to water vapor (moisture) and liquid water. Early applications of the QCM were in air or vacuum to monitor adsorption and deposition processes. Based on the well-known Sauerbrey relationship it was possible to relate frequency changes of the QCM to added mass through the crystal constant ${ }^{146}$. After the development of oscillation circuits to ensure stable operation in liquids ${ }^{147}$, the QCM technique gained more widespread use, especially in electrochemistry ${ }^{148}$ and as a transducer for gravimetric sensors in the biomedical field ${ }^{149}$. With the advent of the quartz crystal microbalance with dissipation (QCM-D) technique, QCM methods can be used to monitor not only the frequency changes associated with mass changes of a thin film on the oscillating crystal, but also the dissipation factor, which provides information on the mechanical properties of thin films ${ }^{150}$.

The dissipation factor, $\mathrm{D}$, which is defined as the reciprocal of the quality factor, Q, of the oscillation of the quartz crystal, represents the ratio of the energy dissipation to the stored energy during an oscillation. Thus, when the oscillation of a quartz crystal is damped by the presence of a lossy material, the dissipation factor will be higher than when the oscillation is not damped. Since immersion of a quartz crystal in a liquid will damp the oscillation, the dissipation factor in liquids is higher than that in air or vacuum. Furthermore, a viscoelastic polymer layer on a QCM will show greater dissipation than a rigid, elastic film. Therefore, the dissipation factor may be used to determine mechanical properties of various materials coated on a quartz crystal in a QCM. 
The QCM-D method has been used to study absorption, swelling kinetics, and mechanical properties of various polymer films and gels ${ }^{151-155}$. Furthermore, temperature dependent QCM-D studies have been performed to study the swelling and conformation changes associated with uncrosslinked, solution polymerized NIPAAm chains grafted to $\mathrm{SiO}_{2}$ coated quartz crystal resonators ${ }^{156-159}$. Based on the frequency and dissipation changes at various temperatures, the authors were able to study the coil to globule transition in the grafted NIPAAm chains; in contrast to the sharp coil to globule transition found in linear NIPAAm chains in solution, the grafted NIPAAm chains showed a continuous transition in the temperature range $20-38^{\circ} \mathrm{C}$.

Plasma polymerization produces randomly crosslinked polymeric networks; properties of plasma polymers (e.g., crosslink density) can be controlled by variation of reactor conditions such as substrate temperature, reactor operating pressure, applied RF power, and precursor flow rates. Based on the discussion in Chapter 3, it is clear that NIPAAm thin films obtained under different process conditions of reactor pressure and substrate temperature show different chemical bonding characteristics. In particular, films obtained at higher reactor pressures and lower substrate temperatures show strong intermolecular hydrogen bonding and a high percentage loss in film thickness upon exposure to liquid water possibly due to lack of adequate crosslinking. Conversely, NIPAAm films obtained at lower reactor pressures and higher substrate temperatures show negligible intermolecular hydrogen bonding and a negligible or positive change in film thickness upon exposure to water. 
Previous reports on plasma polymerized NIPAAm films have focused on characterizing the thermoresponsive properties through a variety of surface analytical techniques ${ }^{19,20,70,71}$, but details on the bulk material properties such as water and moisture uptake characteristics were not provided. Specifically, we are not aware of any QCM-D studies of water and moisture uptake characteristics of plasma polymerized NIPAAm films. Furthermore, to our knowledge, no attempts were made to vary the crosslink density of plasma polymerized hydrogel films by changing the reactor processing conditions. In this study, the water and moisture uptake characteristics of NIPAAm films prepared under different reactor conditions are correlated to their physicochemical properties through QCM-D studies. Furthermore, the existence of an LCST type of transition will be confirmed. Although the existence of the LCST transition in plasma polymerized NIPAAm films was shown through temperature dependent contact angle measurements in Chapter 3, it was not possible to comment on whether the transition occurred in the bulk of the thin film; the QCM-D studies are useful in this regard since temperature dependent water uptake values, with lower water uptake capacities at higher temperatures will indicate that the bulk of the thin films are capable of exhibiting different affinities to water. Knowledge of moisture and water uptake characteristics of plasma polymerized hydrogel films will assist in the development of chemical/biomolecule sensitive films for sensor applications. Since plasma-deposited NIPAAm films are deposited from the vapor phase, the necessary cross-linking and adhesion properties may be built into the films during deposition. With this approach, the 
incorporation and integration of these films into sensors and other electronic devices is

facilitated relative to grafting or spin-casting methods of film formation ${ }^{104,160}$.

\subsection{Experimental section}

Plasma polymerized NIPAAm thin films were prepared in a $13.56 \mathrm{MHz}$, parallel plate reactor at temperatures of either $120^{\circ}$ or $175^{\circ} \mathrm{C}$ and pressures of either 93.3 or 133.3 Pa. A 1:1 volumetric mixture of argon and NIPAAm vapor was fed into the reactor. Details of the reactor configuration, thin film deposition methods and film characterization have been published previously ${ }^{21,27}$, and also given in Chapter 3 . To investigate the water uptake and moisture sorption characteristics of the NIPAAm films obtained under different process conditions, QCM-D studies were performed at various temperatures between 20 and $40^{\circ} \mathrm{C}$.

Plasma polymerized NIPAAm thin films were deposited onto quartz crystal sensors (QCM) (Q-Sense, Newport Beach, CA) coated with $50 \mathrm{~nm}$ silicon/silicon dioxide $\left(\mathrm{Si} / \mathrm{SiO}_{2}\right)$ to study their water uptake and moisture sorption characteristics. Immediately after deposition, the films were rinsed in deionized water three times to remove the uncrosslinked monomers or polymer chains. Subsequently, the film thickness was measured using variable angle spectroscopic ellipsometry. All NIPAAm films used in this study had a nominal thickness of $100 \mathrm{~nm}$. 
The quartz crystals are thin, circular discs made from AT-cut quartz and sandwiched between two gold electrodes. Since quartz is piezoelectric, application of an AC voltage across the disc introduces a thickness shear mode resonant wave in the ATcut crystal; based on the cut of the quartz crystal and the dimensions, crystals can be set to resonate at various frequencies. In the QCM-D technique, the quartz crystal is electrically driven to oscillate at its fundamental frequency of $5 \mathrm{MHz}$ or at one of the three overtones at $15(\mathrm{n}=3), 25(\mathrm{n}=5)$, or $35(\mathrm{n}=7) \mathrm{MHz}$; the dissipation factor is determined by measuring the exponential decay constant of the resonant wave after the drive is short circuited. In the case of rigid overlayers on the quartz crystal, the amount of mass coupled to the oscillating crystal can be determined by applying the Sauerbrey relationship, which states that the change in mass $(\Delta \mathrm{m})$, or mass coupled to the crystal, is directly proportional to the change in resonant frequency $(\Delta \mathrm{f})$ :

$\Delta m=-\frac{C \Delta f}{n}$

where, $C=17.7 \mathrm{ng} / \mathrm{Hz}-\mathrm{cm}^{-2}$ for a $5 \mathrm{MHz}$ crystal, and is also known as the crystal constant; $n=3,5$, or 7 , is the overtone number.

From Eq. (4.1), it is clear that for a rigid overlayer, the frequencies of different overtones scale by the overtone number. Consequently, when the frequency values are normalized by the overtone number, they would collapse to a single set of values. However, when the material coupled to the quartz crystal is viscoelastic, the frequencies do not scale linearly with the overtone number, and the dissipation factor is increased relative to a rigid overlayer. As mentioned earlier, the dissipation factor may be expressed as the ratio of the loss modulus $\left(E_{\text {loss }}\right)$ to the storage modulus $\left(E_{\text {storage }}\right)$, and thus is an indicator of the softness of the overlayer. 


$$
D=\frac{E_{\text {loss }}}{2 \pi E_{\text {storage }}}
$$

where $\mathrm{D}$ is the dissipation factor.

The behavior of plasma polymerized NIPAAm thin films in liquid water was monitored at different temperatures through frequency and dissipation changes in the QAFC 302 flow cell of the QCM-D apparatus. First, a NIPAAm coated quartz crystal was mounted in the QCM-D flow cell; the temperature of the flow cell was allowed to stabilize at $20 \pm 0.1^{\circ} \mathrm{C}$; subsequently, deionized (DI) water flow was initiated. After this initial purge, the flow of DI water was terminated and the NIPAAm coated QCM exposed to a constant quantity of liquid water $(\sim 80 \mu \mathrm{L})$ for the remainder of the experiment. Since the flow of DI water caused a temperature change, additional time was allowed for the temperature to stabilize at the set point. After temperature stabilization, frequency and dissipation data were acquired. Data acquisition continued at a given temperature until stable readings of the frequency and dissipation data were attained. Similarly, frequency and dissipation data were acquired at other temperatures between 18 and $40{ }^{\circ} \mathrm{C}$. At each temperature, the time required for the frequency and dissipation values to stabilize was approximately twenty minutes.

Moisture sorption experiments were also performed in the QAFC 302 flow cell at three different temperatures: $18{ }^{\circ} \mathrm{C} \pm 0.1{ }^{\circ} \mathrm{C}, 25^{\circ} \mathrm{C} \pm 0.1{ }^{\circ} \mathrm{C}$, and $35^{\circ} \mathrm{C} \pm 0.1^{\circ} \mathrm{C}$. After loading a NIPAAm coated quartz crystal into the QCM-D apparatus, the flow cell base and the crystal were brought to the set temperature. Subsequently, the flow cell was 
purged with dry $\mathrm{N}_{2}$ for 30 min to remove background moisture contained in the NIPAAm films. At the end of $30 \mathrm{~min}$ and while dry $\mathrm{N}_{2}$ was still flowing, data acquisition was initiated. Dry $\mathrm{N}_{2}$ flow continued for an additional five minutes before the QCM was exposed to ambient humidity (relative humidity $\sim 40 \%$ ). The data values recorded in the dry $\mathrm{N}_{2}$ environment served as the baseline for subsequent measurements. Frequency and dissipation values of the NIPAAm coated quartz crystal were recorded in ambient humidity for approximately $60 \mathrm{~min}$ after the $\mathrm{N}_{2}$ flow was terminated. At this point, the rate of decrease in the frequency due to moisture uptake had slowed to less than $2 \mathrm{~Hz}$ every 15 min. Following exposure to ambient humidity, the quartz crystal was exposed to dry $\mathrm{N}_{2}$ to remove the sorbed moisture from the NIPAAm films.

All frequency and dissipation factor changes reported in this study were determined by subtracting the value of frequency or dissipation of the bare crystal from the value measured for the NIPAAm coated quartz crystal at each temperature. Since the frequency and dissipation factor of the bare quartz crystal can vary with temperature, the values of frequency and dissipation were determined at various temperatures between 20 and $40{ }^{\circ} \mathrm{C}$ prior to depositing NIPAAm on the quartz crystal resonators.

\subsection{Results and Discussion}

Water and moisture uptake measurements of plasma polymerized NIPAAm thin films were obtained for films deposited under four different conditions of substrate temperature and reactor pressure: $120^{\circ} \mathrm{C}, 133.3 \mathrm{~Pa}$ (process condition $\mathrm{A}$ ), $120^{\circ} \mathrm{C}, 93.3 \mathrm{~Pa}$ (process condition (B), $175^{\circ} \mathrm{C}, 133.3 \mathrm{~Pa}$ (process condition $\mathrm{C}$ ), and $175^{\circ} \mathrm{C}, 93.3 \mathrm{~Pa}$ 
(process condition D). However, due to film dissolution during water rinsing, only results from films prepared using the latter three process conditions are presented below. That is, when NIPAAm thin films prepared at a substrate temperature of $120^{\circ} \mathrm{C}$, and a reactor pressure of $133.3 \mathrm{~Pa}$ are exposed to liquid water to remove uncrosslinked chains and monomer from the network, the film thickness decreases to $\sim 15 \%$ of its original value, apparently due to the lack of adequate crosslinking. The reduction in film thickness causes considerable roughening of the film surface; as a result, it can no longer be considered a homogeneous overlayer on the quartz crystal. Thus, thickness measurements with variable angle spectroscopic ellipsometry were not possible due to scattering from the rough surface of the NIPAAm films. Furthermore, QCM-D data from such films may not be reliable since the increased surface area of the film can enhance water retention.

\subsubsection{Water uptake measurements}

The normalized frequency and dissipation values for overtones 3, 5, and 7 of quartz crystals coated with NIPAAm prepared using process conditions B, C, and D are shown in Figs. 4.1, 4.2, and 4.3 respectively. Since the trend in the fundamental frequency and dissipation factor with temperature was inconsistent with that observed in the case of $3^{\text {rd }}$, $5^{\text {th }}$, and $7^{\text {th }}$ overtones, they were not included in the following analysis. Indeed, such behavior, believed to be caused by poor energy trapping due to the small size of the crystal, has been reported by other investigators ${ }^{154,161}$. Furthermore, in a previous study, the authors use only the $3^{\text {rd }}$ overtone of the frequency and dissipation to draw conclusions

on the conformation changes in poly (NIPAAm) chains with temperature ${ }^{157,159}$. Since 
the normalized frequency values for the three different overtones do not collapse to a single set of values, the plasma polymerized NIPAAm films are frequency dependent, and hence may be viscoelastic. Therefore, the Sauerbrey formalism to determine water uptake capacities is not applicable.

Figures 4.1, 4.2, and 4.3 indicate that frequency and dissipation values of the NIPAAm films are temperature dependent. The values of $|\Delta \mathrm{f}|$ are higher at lower temperatures, and decrease as temperature increases. This indicates that the NIPAAm films take up water at the lower temperatures and reject it at higher temperatures. In the temperature range studied, the transition between the hydrophilic and hydrophobic states appears continuous and essentially reversible. Similarly, the values of $\Delta \mathrm{D}$ decrease with an increase in temperature. The trend in dissipation values supports the inference from $|\Delta f|$ values: when the NIPAAm films are hydrophilic at lower temperatures, increased water absorption causes the films to show greater deviation from a pure elastic behavior, resulting in greater dissipation. At higher temperatures, the observed lower dissipation values are consistent with a more rigid polymeric network with reduced water content as indicated by the frequency changes. The overall change in $\Delta \mathrm{f}$ with temperature, as a result of the hydration/dehydration of the NIPAAm network, is approximately $150 \mathrm{~Hz}$, and is comparable to the change observed when NIPAAm chains were grafted to a QCM surface; however, the overall change in $\Delta \mathrm{D}$ with temperature is higher than that reported in previous investigations ${ }^{157,158}$. 

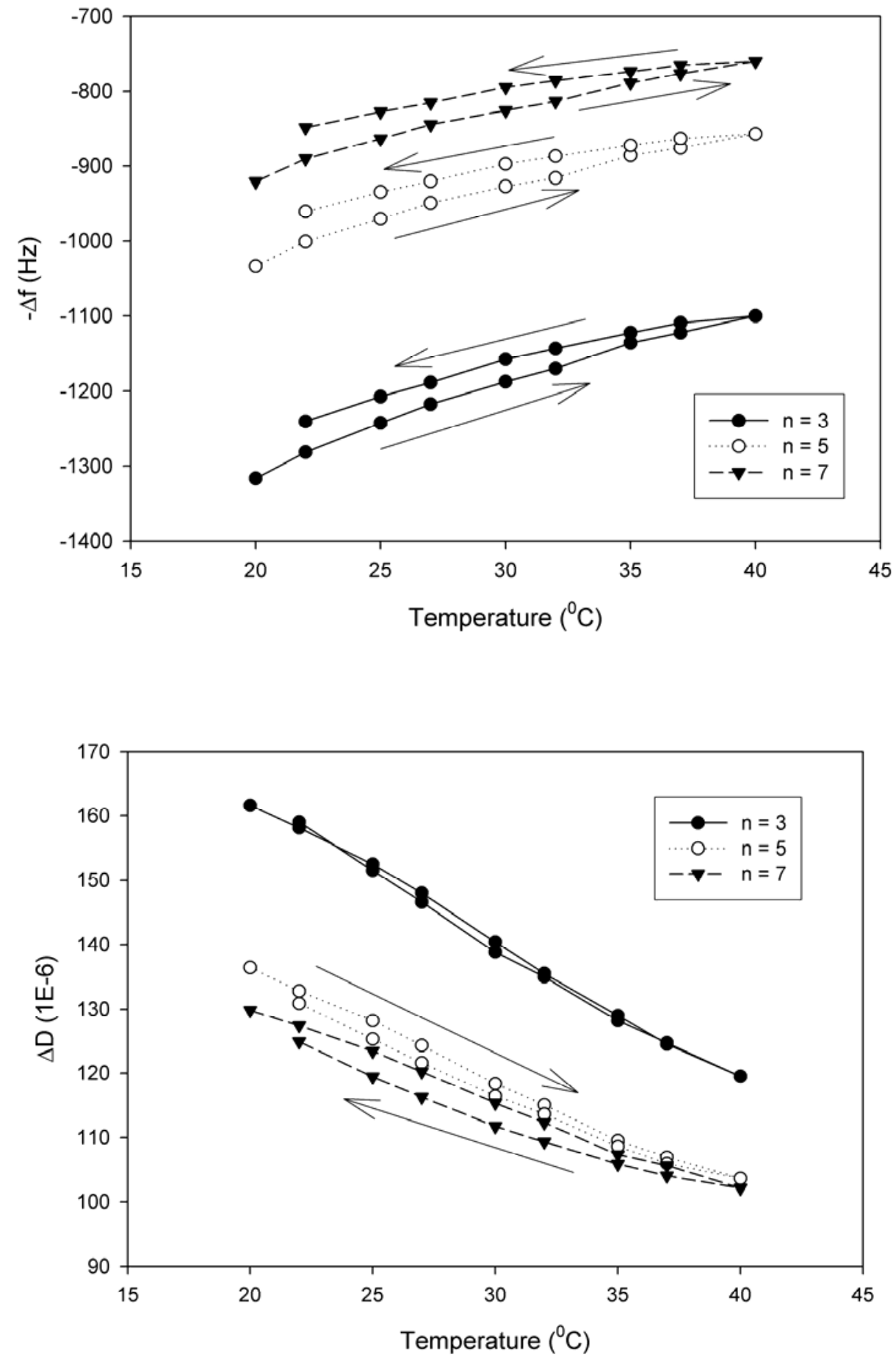

Figure 4.1 Variation of $-\Delta \mathrm{f}$ (top) and $\Delta \mathrm{D}$ (bottom) with temperature for NIPAAm films prepared using process condition $\mathrm{B}$ (Substrate temperature $=120^{\circ} \mathrm{C}$; Reactor pressure $=$ 93.3 Pa). 

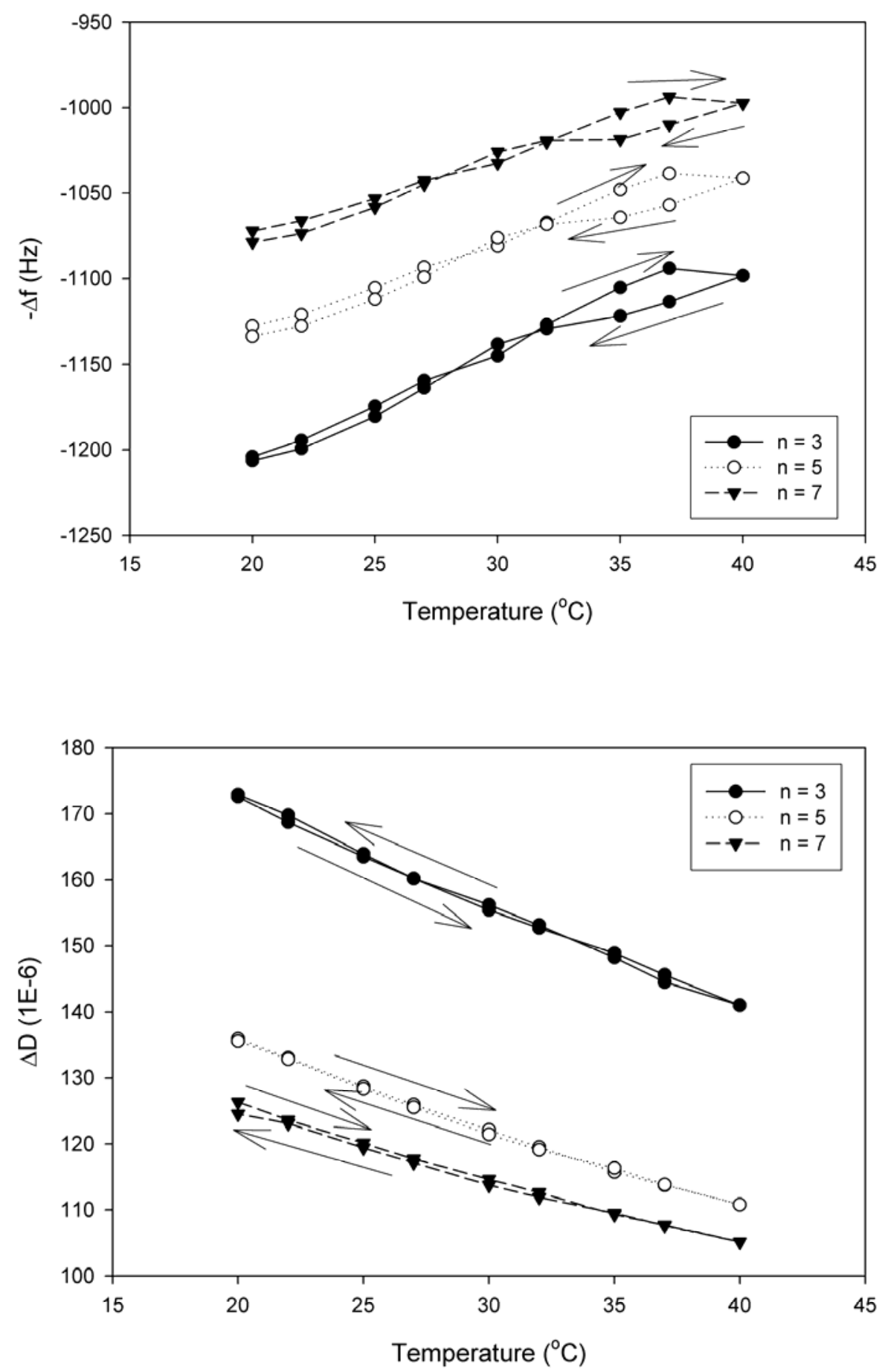

Figure 4.2 Variation of $-\Delta \mathrm{f}$ (top) and $\Delta \mathrm{D}$ (bottom) with temperature for NIPAAm films prepared using process condition $\mathrm{C}$ (Substrate temperature $=175^{\circ} \mathrm{C}$; Reactor pressure $=$ 133.3 Pa). 

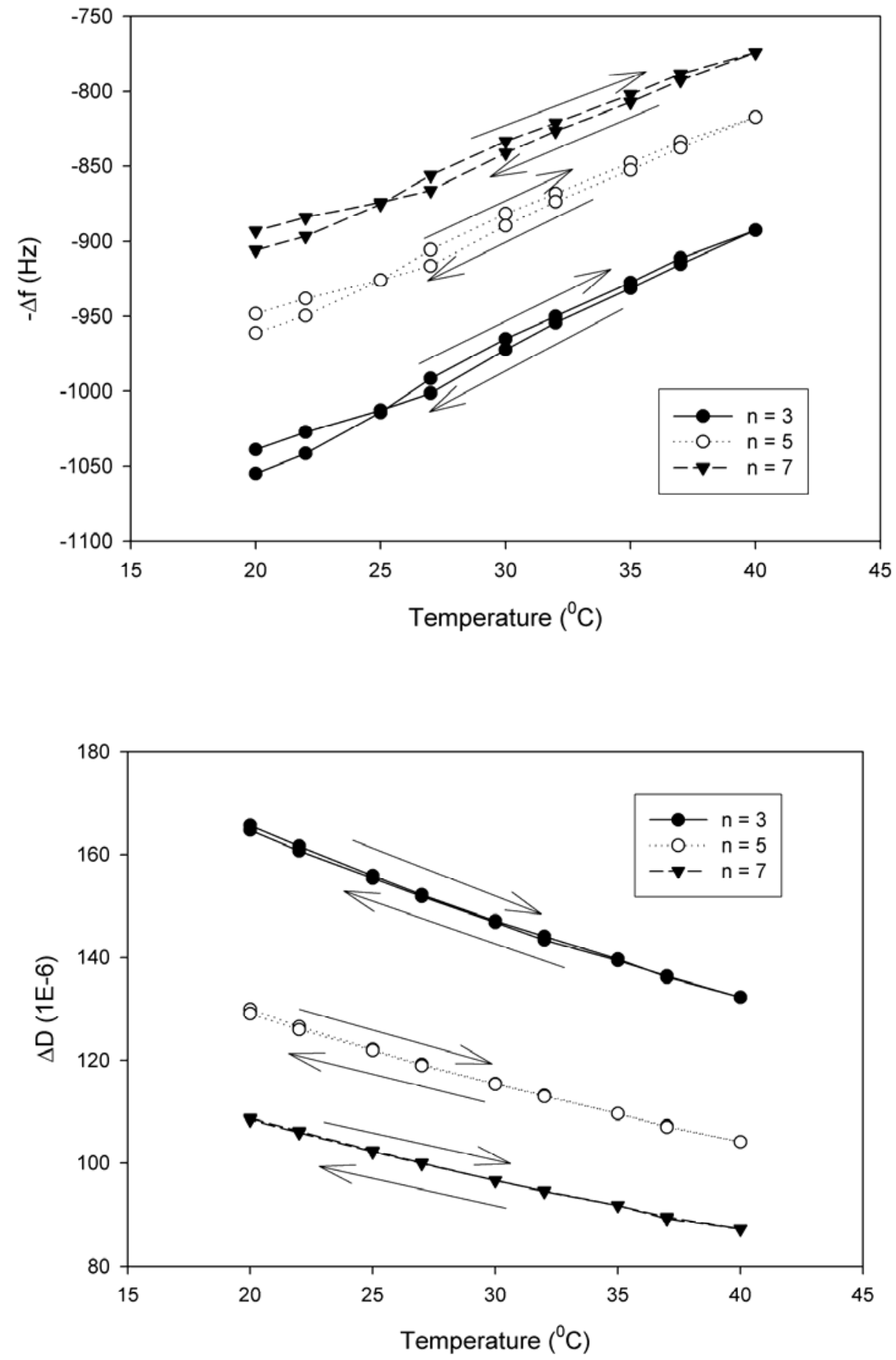

Figure 4.3 Variation of $-\Delta \mathrm{f}$ (top) and $\Delta \mathrm{D}$ (bottom) with temperature for NIPAAm films prepared using process condition $\mathrm{D}\left(\right.$ Substrate temperature $=175^{\circ} \mathrm{C}$; Reactor pressure $=$ 93.3 Pa). 
Previous studies have demonstrated that plots of $\Delta \mathrm{D}$ versus $-\Delta \mathrm{f}$ or $-\Delta \mathrm{D} / \Delta \mathrm{f}$ versus temperature display changes in D (possibly due to structure or conformation changes), and $\mathrm{f}$ (due to changes in coupled mass), and therefore permit the two processes to be monitored simultaneously ${ }^{162}$. The importance of such approaches has been demonstrated for surface grafted poly (NIPAAm) chains; although the variation of $-\Delta \mathrm{f}$ and $\Delta \mathrm{D}$ with temperature was continuous, plots of $\Delta \mathrm{D}$ versus $-\Delta \mathrm{f}$ and $-\Delta \mathrm{D} / \Delta \mathrm{f}$ versus temperature revealed changes in the slope at approximately $30{ }^{\circ} \mathrm{C}$, close to the well-accepted LCST temperature of NIPAAm chains in water ${ }^{156-159}$. Based on these changes in slope, swelling and collapse behavior of poly (NIPAAm) chains were inferred.

Figure 4.4 shows the variation of $-\Delta \mathrm{D} / \Delta \mathrm{f}$ (based on the $3^{\text {rd }}$ overtone) with temperature for plasma polymerized NIPAAm films deposited under process conditions $B, C$, and D. Except for minor changes in the slopes, variation of $-\Delta D / \Delta f$ appears continuous with temperature, suggesting that hydration/dehydration of the NIPAAm network and the corresponding mechanical property changes occur simultaneously. At higher temperatures, $-\Delta \mathrm{D} / \Delta \mathrm{f}$ is reduced relative to that at lower temperatures, suggesting that the dissipation per unit mass is lower at higher temperatures. This is consistent with the LCST type of transition expected for NIPAAm films. Furthermore, at all temperatures studied, the value of $-\Delta \mathrm{D} / \Delta \mathrm{f}$ was highest for NIPAAm films prepared under process condition $\mathrm{D}$, indicating that these films show the highest dissipation per unit mass of coupled water, possibly due to greater water uptake relative to that of the other films. 


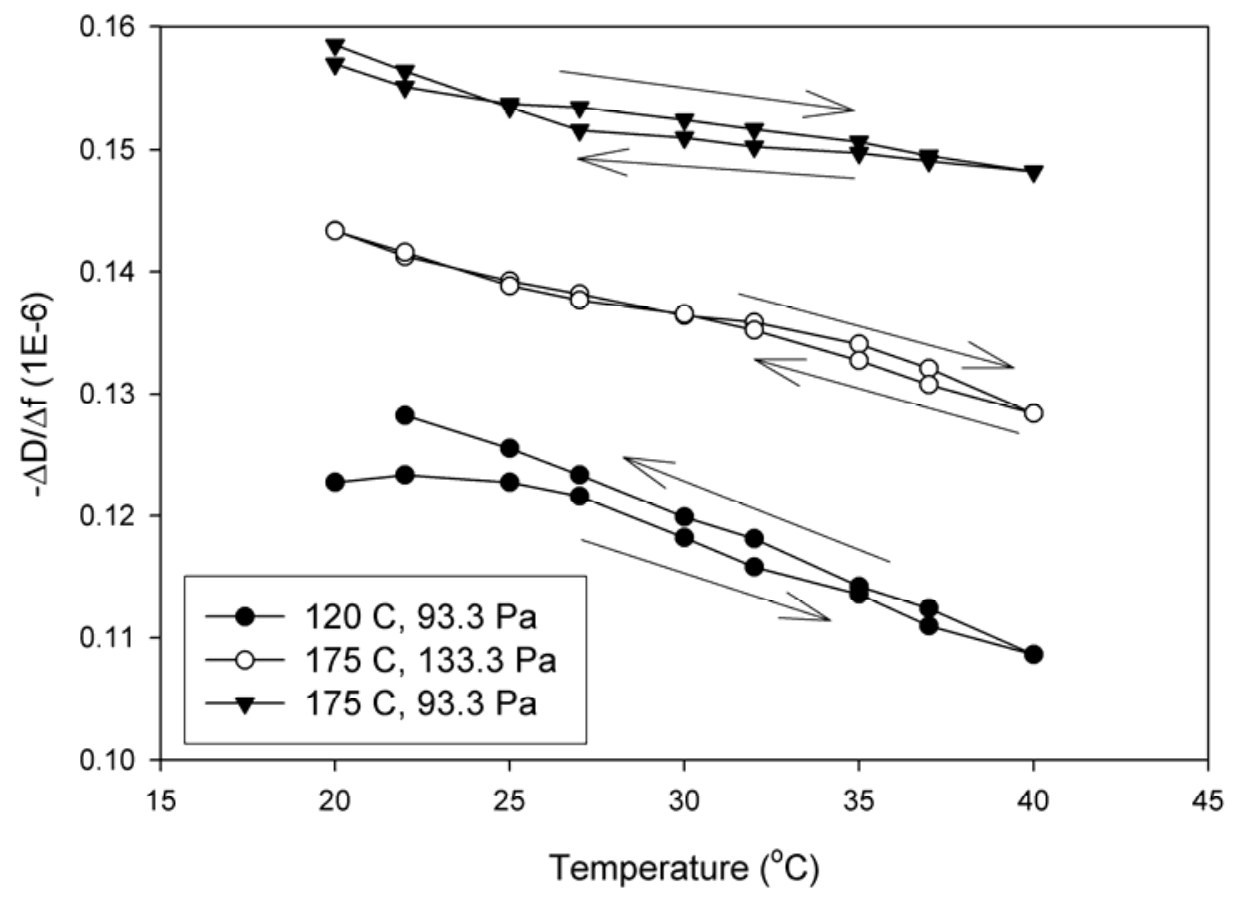

Figure 4.4 Variation of $-\Delta \mathrm{D} / \Delta \mathrm{f}(1 \mathrm{E}-6)$ with temperature for NIPAAm films prepared using different reactor conditions.

The results from Figure 4.4 are in contrast to previous results reported for surface grafted poly (NIPAAm) chains ${ }^{157}$, where $-\Delta \mathrm{D} / \Delta \mathrm{f}$ was constant with temperature in the range of $20-30{ }^{\circ} \mathrm{C}$, but increased at $\mathrm{T}>30^{\circ} \mathrm{C}$. An explanation for such a transition was not offered. However, in a different study on conformation changes in adsorbed pNIPAAm chains on QCM surfaces, the variation of $-\Delta \mathrm{D} / \Delta \mathrm{f}$ with temperature was similar to the results presented here in that $-\Delta \mathrm{D} / \Delta \mathrm{f}$ varied continuously with temperature; in addition, the range of values obtained for $-\Delta \mathrm{D} / \Delta \mathrm{f}$ in our study $(\sim 0.16-0.10)$ match those obtained in the previous study ${ }^{156}$. The values of $-\Delta \mathrm{D} / \Delta \mathrm{f}$ obtained in this study are also in close agreement with the theoretical value of 0.13 predicted for the $3^{\text {rd }}$ overtone, 
based on a quantitative analysis of the oscillation of the quartz crystal resonator in liquids ${ }^{163,164}$.

Over the temperature range studied in the current investigation, the existence of a continuous rather than a discontinuous transition for the plasma polymerized NIPAAm films is plausible. In fact, as shown in Chapter 3, temperature dependent sessile contact angle measurements on the plasma polymerized NIPAAm films showed the existence of a broad transition with transition temperatures (at the inflection points of the sigmoidal curves) below $20^{\circ} \mathrm{C}^{21}$. Since plasma polymerization is likely to produce a randomly crosslinked network with a broad range of polymer chain lengths between crosslinks, a wide spectrum of collapse rates may be expected. In addition to the continuous transition that is expected in inhomogeneous networks ${ }^{142}$, the constraint due to adhesion of the films to the substrate causes continuous transitions that are controlled by kinetic rather than purely thermodynamic processes ${ }^{165}$.

\subsubsection{Moisture sorption experiments}

The results from moisture sorption experiments performed on NIPAAm films prepared using conditions B, C, and D are shown in Figs. 4.5 (a) - (c). Specifically, Figs. 4.5 (a) - (c) show the variation of the swelling factor, Q, with time ${ }^{1 / 2}$ at three different temperatures. The swelling factor, $\mathrm{Q}$, at a given temperature may be defined ${ }^{166}$ as the ratio of the frequency of NIPAAm coated quartz crystal exposed to ambient humidity, to the frequency of NIPAAm coated quartz crystal measured in dry $\mathrm{N}_{2}$; both frequencies are expressed relative to that of the bare crystal. The swelling factor, Q, was calculated for 
all three process conditions from changes in the $3^{\text {rd }}$ overtone of the fundamental frequency $(\sim 15 \mathrm{MHz})$ of the QCM data. Since the normalized frequencies for all overtones collapsed to that of the fundamental frequency, it is possible to use the Sauerbrey relationship to calculate the moisture uptake capacity.

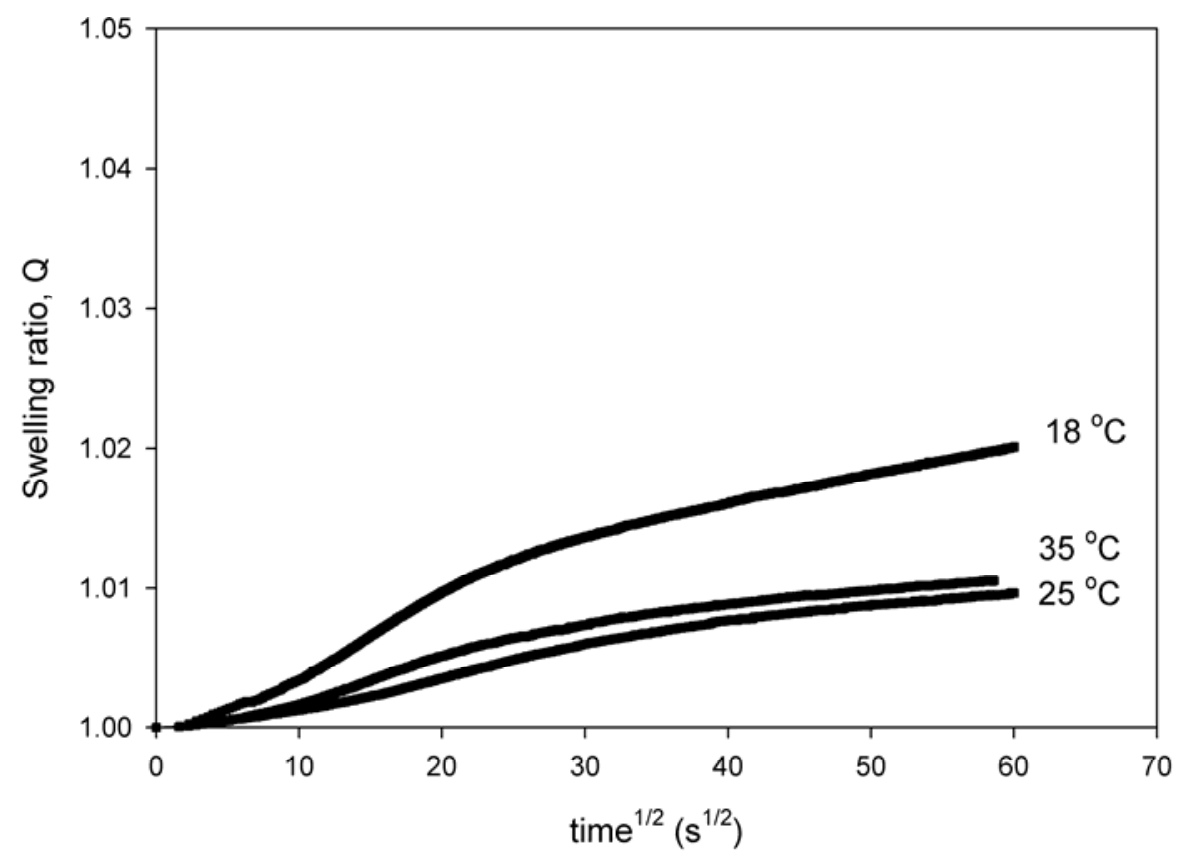

Figure 4.5 (a) Moisture absorption kinetics from ambient air ( $\sim 40 \%$ relative humidity) for NIPAAm film prepared using process condition B (Substrate temperature $=120^{\circ} \mathrm{C}$; Reactor pressure $=93.3 \mathrm{~Pa}$ ). 

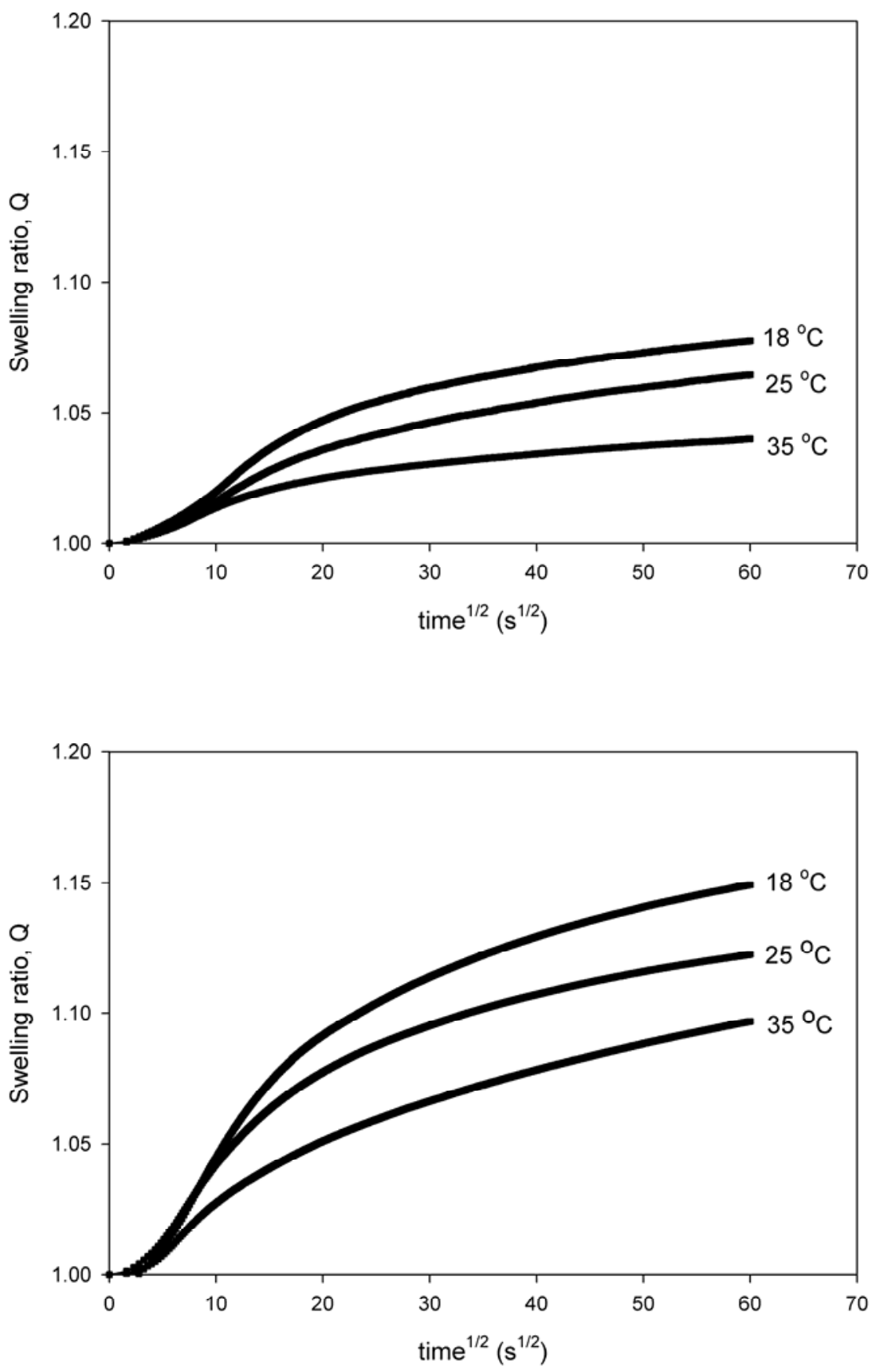

Figure 4.5 continued Moisture absorption kinetics from ambient air ( $\sim 40 \%$ relative humidity) for NIPAAm films prepared using: (b) process condition C (Substrate temperature $=175^{\circ} \mathrm{C}$; Reactor pressure $=133.3 \mathrm{~Pa}-$ top); (c) process condition $\mathrm{D}$ (Substrate temperature $=175^{\circ} \mathrm{C}$; Reactor pressure $=133.3 \mathrm{~Pa}-$ bottom). 
Figures 4.5 (a) - (c), demonstrate that the moisture sorption process in NIPAAm thin films follows non-Fickian kinetics since the initial portion of the integral sorption curve is not linear with the square root of time. Furthermore, there is no definitive saturation level in the sorption curve; at the end of sixty minutes, the sorption process appears to reach a pseudo-equilibrium state. The observed non-linear sorption process is consistent with sigmoidal behavior at short times and two-stage sorption at longer times $^{167}$. Sigmoidal sorption processes occur due to a time dependence of the penetrant surface concentration and slow establishment of equilibrium at the surface ${ }^{168}$. The variable surface concentration (VCS) model, which assumes a constant diffusivity for Fickian diffusion and a variable surface concentration, gives a satisfactory description of the sigmoidal behavior. The two-stage sorption is believed to occur due to polymer relaxation effects ${ }^{169}$.

In addition to the kinetics of the sorption process, it is important to consider the moisture uptake capacities of the NIPAAm films at different temperatures. Values of the swelling ratio, Q, at the end of the sixty minute sorption process, are plotted in Figure 4.6 as a function of the process condition employed to prepare NIPAAm films; the error bars in Figure 4.6 are fractional errors in the swelling ratio computed from systematic errors in the measured frequency values. Uncertainty in the measured frequency values was determined by measuring the frequency by mounting and dismounting the quartz crystal resonator in the flow cell several times. Indeed, other investigators have reported on the introduction of such systematic errors in the measurement of frequency values ${ }^{154,161}$. 


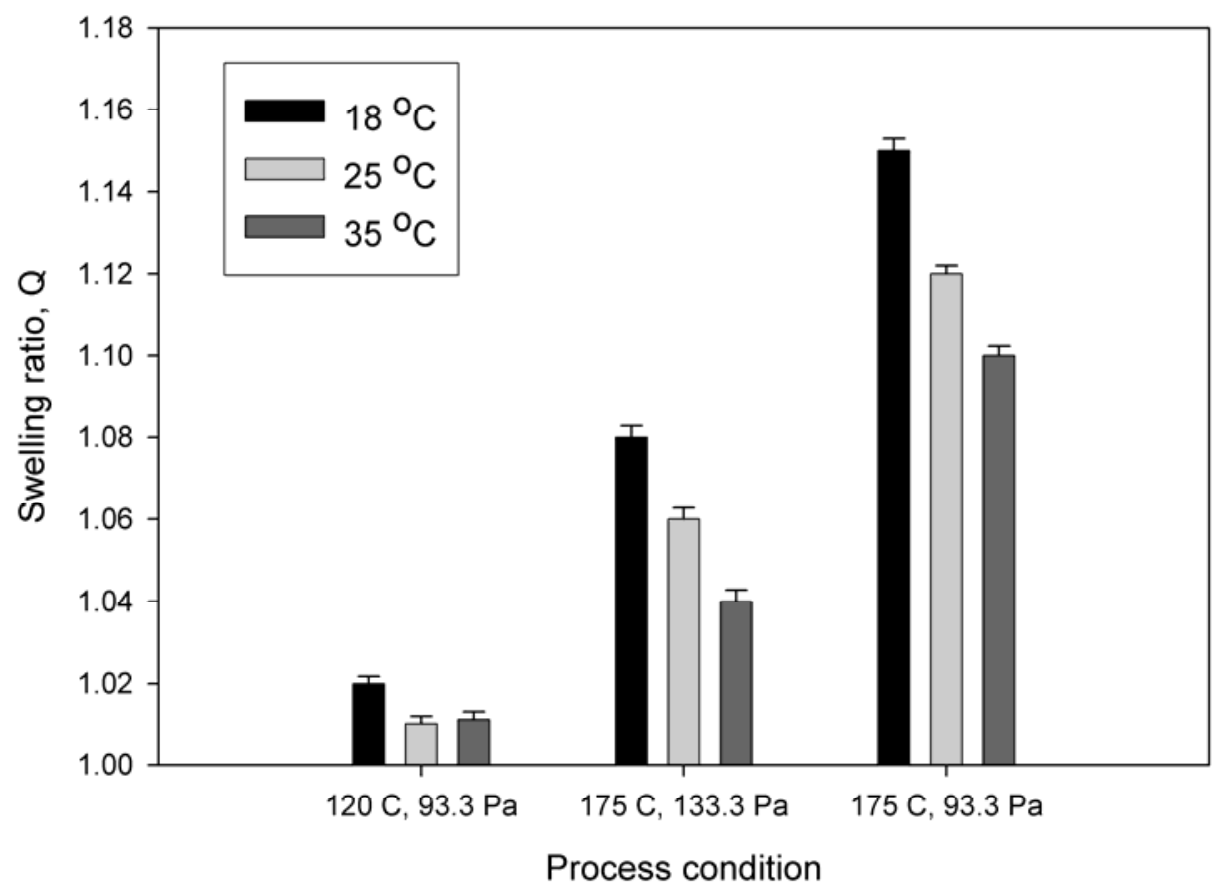

Figure 4.6 Comparison of moisture uptake capacities of NIPAAm films prepared using different reactor conditions, through swelling ratio, $Q$.

From Figure 4.6, it is clear that for NIPAAm films prepared at $175^{\circ} \mathrm{C}$, the sorption capacity of the films decreases continuously from $18{ }^{\circ} \mathrm{C}$ to $35^{\circ} \mathrm{C}$, thus confirming the thermoresponsive properties of the films. However, for the NIPAAm film obtained at $120^{\circ} \mathrm{C}, 93.3 \mathrm{~Pa}$, while the sorption capacity is highest at $18^{\circ} \mathrm{C}$, the sorption capacities at $25^{\circ} \mathrm{C}$ and $35^{\circ} \mathrm{C}$ differ only by $0.1 \%$; the value of $\mathrm{Q}$ at $25^{\circ} \mathrm{C}$ is 1.01 , whereas at $35^{\circ} \mathrm{C}$ it is 1.011 . This discrepancy may be due to the difficulty involved in accurately determining swelling ratios that are already small (swelling ratio $=1.02$ at 18 $\left.{ }^{\circ} \mathrm{C}\right)$.

Furthermore, Figure 4.6 shows that at all temperatures, the swelling ratio is highest for NIPAAm films prepared using condition D. At first glance, this result seems 
counterintuitive because the results shown in Chapter 3 suggested that due to the greater electron and ion energies in the plasma reactor at increased substrate temperatures and low reactor pressures, the resulting NIPAAm films possess higher crosslink density. In fact, when such films are exposed to liquid water immediately after the deposition to extract the uncrosslinked chains, there is either a positive or negligible change in the film thickness; in contrast, similar studies on NIPAAm films deposited at lower substrate temperatures and higher reactor pressures resulted in large negative changes in the film thickness. Since films with greater crosslink density are able to exert greater elastic forces, they resist the expansion of the polymer matrix due to water/moisture uptake. Therefore, films with greater crosslink density should show lower swelling ratios.

The results in Figure 4.6 may be explained on the basis of the chemical bonding characteristics of the plasma-deposited NIPAAm films. It was previously shown in Chapter 3 that based on the positions of amide I and II bands in the Fourier transform infrared spectra, deposition at higher substrate temperatures and lower reactor pressures generate NIPAAm films with weak hydrogen bonding characteristics ${ }^{21}$. Conversely, NIPAAm films deposited at lower substrate temperatures and higher reactor pressure display stronger hydrogen bonding. The existence of strong intermolecular hydrogen bonding can hinder the ingress of moisture or water in the polymeric network since the polar groups are not available for hydrogen bonding with the penetrant.

Indeed, the effect of hydrogen bonding on moisture and water uptake capacity has been studied in other polymeric systems. Due to strong intermolecular hydrogen bonding 
at $\mathrm{pH}$ values less than the critical $\mathrm{pH}$ of a crosslinked network of polyvinyl pyrrolidinonepolyacrylic acid, the swelling ratio of the network was considerably lower relative to the swelling ratio in a solution with $\mathrm{pH}$ higher than the critical $\mathrm{pH}$ of the network ${ }^{170}$. At higher values of $\mathrm{pH}$, due to ionization of the carboxylic acid groups of acrylic acid, the intermolecular hydrogen bonding diminishes and thus causes greater swelling.

Other studies have demonstrated that although a decrease in water uptake capacity with increase in crosslinking in partially crosslinked hydrogels (based on carboxymethylcellulose) is observed, the completely crosslinked hydrogel showed the greatest water uptake capacity ${ }^{171}$. Furthermore, the partially crosslinked hydrogels showed $\mathrm{pH}$ dependent swelling behavior due to the ionization of carboxylic acid groups, whereas the $100 \%$ crosslinked hydrogel did not. This behavior has been attributed to the effects of hydrogen bonding; the partially crosslinked hydrogels possessed considerable hydrogen bonding which diminished the water uptake capacity. On the other hand, the completely crosslinked hydrogel showed negligible hydrogen bonding and thus was able to swell to a greater extent. Again, evidence of hydrogen bonding in the hydrogels was obtained from amide I and II bands of the FTIR spectra.

Thus, we conclude that plasma-polymerized NIPAAm films with greater crosslink density have greater moisture uptake capacity due to the lower extent of intermolecular hydrogen bonding. Conversely, NIPAAm films with a high degree of intermolecular hydrogen bonding show smaller moisture uptake capacity. However, it is important to note that the dry $\mathrm{N}_{2}$ purge, used to dehydrate NIPAAm films prior to moisture absorption 
studies may not have removed all the moisture trapped in the polymer network. It is therefore possible that the NIPAAm film from process condition B possessed

irremovable water ${ }^{172}$, thereby preventing the uptake of any additional moisture. This conclusion is in contrast to NIPAAm films prepared using process condition D, which may contain less residual moisture after the dry $\mathrm{N}_{2}$ purge, and hence may allow a greater moisture uptake. In any case, it is clear that hydrogen bonding controls the moisture uptake capacity of the plasma-polymerized NIPAAm films.

\subsection{Conclusions}

Liquid water and moisture uptake characteristics of plasma polymerized NIPAAm films were studied between 20 and $40^{\circ} \mathrm{C}$ using the QCM-D technique. Measurements in liquid water indicated that the NIPAAm films are viscoelastic because they show frequency dependent response. In the temperature range studied, $\Delta \mathrm{f}$ and $\Delta \mathrm{D}$ varied continuously with temperature such that the values of $|\Delta f|$ and $\Delta D$ were higher at higher temperatures, thereby confirming the existence of an LCST type of transition. Moisture absorption studies showed that plasma polymerized NIPAAm films followed anomalous, non-Fickian kinetics. Intermolecular hydrogen bonding controlled the moisture uptake capacity; NIPAAm films with a high degree of intermolecular hydrogen bonding exhibited lower moisture uptake capacities. 


\section{CHAPTER 5}

\section{MICROSTRUCTURE CHARACTERIZATION}

\subsection{Introduction}

As described in chapters 3 and 4, plasma polymerized NIPAAm thin films prepared under different reactor conditions appear to possess different crosslink densities, and therefore different microstructures and free volumes. Specifically, information provided by various diagnostic techniques was analyzed to draw several indirect inferences regarding the microstructure of plasma polymerized NIPAAm films. Using a more direct approach, the microstructure of plasma polymerized NIPAAm films were investigated through positron annihilation lifetime spectroscopy (PALS) and Doppler broadening energy spectroscopy (DBES); some preliminary results are presented in this chapter.

Positrons are the anti-particles of electrons, and thus have the same mass and spin as an electron, but opposite charge and magnetic moment. In vacuum, positrons have a long lifetime $\left(\sim 10^{21}\right.$ years $)$, but they are shortlived in condensed matter $\left(\sim 10^{-11} \mathrm{sec}\right)$. Since positrons do not alter material properties during their short lifetime, they are wellsuited for non-destructive probing of condensed matter. When energetic positrons enter a material, they are quickly thermalized within a few picoseconds due to conduction electron or phonon scattering. The implantation depth of positrons depends on their energy and is found to vary as $\mathrm{E}^{1.6}$, where $\mathrm{E}$ is the energy of the positrons ${ }^{204}$. Positrons interact with condensed matter in a variety of processes as shown in Figure 6.1. After 
thermalization, positrons diffuse into the material and undergo free annihilation, or bind to an electron to form a neutral, hydrogen-like atom, called a positronium (Ps), which also subsequently annihilates. Positroniums exist in either the singlet (para-positronium or p-Ps) or the triplet state (ortho-positronium or o-Ps). In vacuum, the p-Ps has a lifetime of 125 ps $\left(\tau_{1}\right)$, and annihilates to two $\gamma$-photons, whereas the o-Ps has a lifetime of $142 \mathrm{~ns}\left(\tau_{3}\right)$ and annihilates to three $\gamma$-photons. However, in condensed matter, the o-Ps is localized in voids and pores, and has a reduced lifetime of just a few nanoseconds. Furthermore, the o-Ps localized within the free volume in the solid, does not annihilate with the electron bound to the positron, but with electrons of the matter surrounding the free volume in a process known as pick-off annihilation to produce two $\gamma$-photons. The PALS technique is based on the observation that the reduced lifetime of o-Ps trapped in free volumes may be correlated to the void size and shape, whereas the intensity of the gamma rays emitted depends on the quantity of such defects ${ }^{205}$.

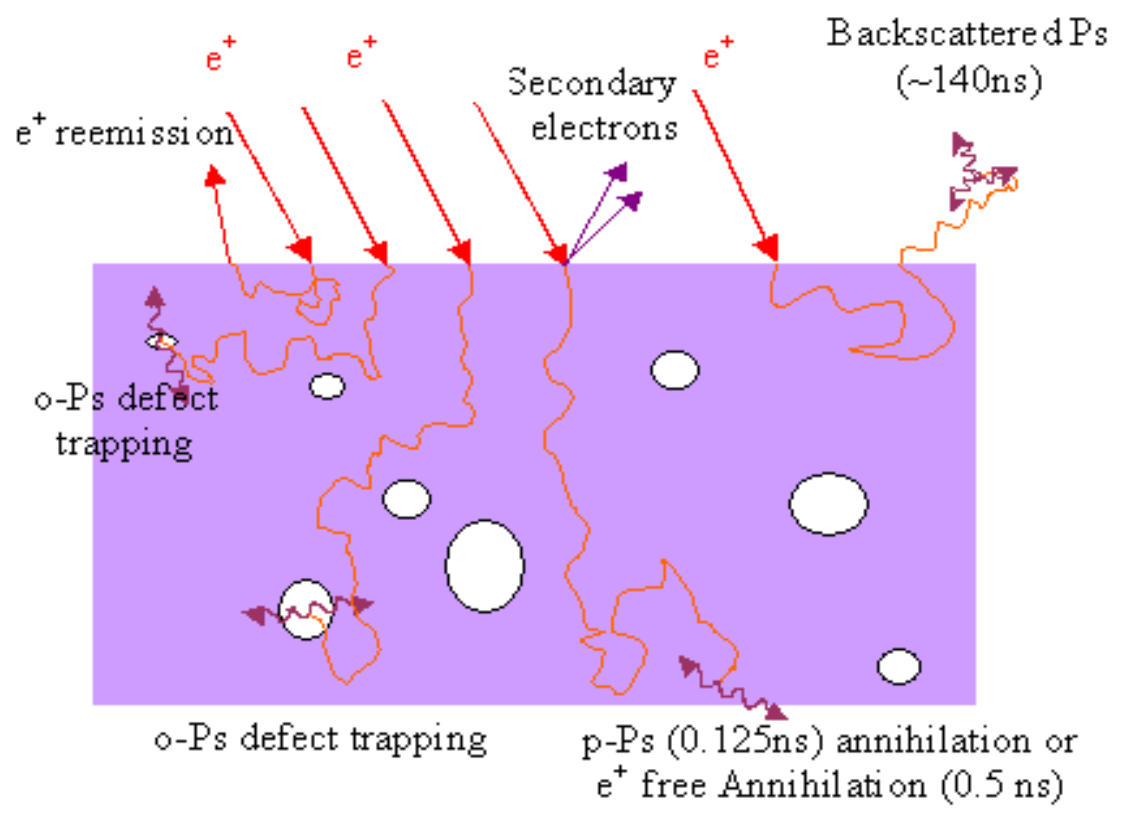

Figure 5.1 Interactions of positron and positronium with condensed matter ${ }^{4}$ 
The energy spectrum of gamma rays emitted upon annihilation of a stationary positronium consists of a narrow peak at $511 \mathrm{keV}$. However, when positrons annihilate with electrons of high momentum, the energy peak at $511 \mathrm{keV}$ is broadened due to the Doppler effect. Doppler broadening of the gamma ray peak is described by the $\mathrm{S}$ parameter, which may be defined as the ratio of integrated counts of gamma photons at $511 \mathrm{keV}$ to the total counts in a window of $\pm 0.53 \mathrm{keV}^{206}$. Thus, the $\mathrm{S}$ parameter defines the sharpness of the annihilation peak at $511 \mathrm{keV}$. By analyzing the gamma rays emitted from the annihilation of a positron, information on the momentum of the electron with which the positron annihilated may be obtained, and thus the environment the positron experienced, and this forms the basis of DBES. When positrons are localized in voids, their probability of annihilation with high momentum, core electrons is lower relative to lower momentum, valence or conduction electrons. Therefore, annihilation of positrons in voids results in a smaller Doppler broadening, which yields a higher S parameter. On the contrary, when positrons are not localized in voids, and therefore annihilate with core electrons of high momentum, the S parameter is lowered due to the Doppler broadening of the annihilation peak. Therefore, the $\mathrm{S}$ parameter is a qualitative indicator of free volume in condensed matter: higher S parameter indicates greater free volume, and viceversa.

Positron annihilation based techniques PALS and DBES have been used to study free volume characteristics of polymeric materials ${ }^{207}$. Additionally, depth profiles of free volume characteristics have been obtained in polymer films by varying the positron 
incident energy ${ }^{208}$. Positron annihilation spectroscopies have been used to study free volumes in thin polymer films as a function of depth and temperature to obtain depth profiles of the glass transition temperature ${ }^{206,209}$. PALS and DBES have been employed to monitor the reduction in free volume in polymeric coatings undergoing photodegradation as a result of exposure to ultra-violet ${ }^{210,211}$ and Xe arc-lamp radiation ${ }^{5,}$ ${ }^{212}$, or weathering ${ }^{213}$. In addition, the increase in crosslink density of a polyurethane coating upon exposure to radiation from a Xe arc-lamp was confirmed through independent, solvent swelling experiments, and correlated to the decrease in the S parameter, and hence free volume $\mathrm{e}^{212}$. Hence, there was an inverse correlation between crosslink density and the S parameter. Additionally, PALS and DBES have been used to study the size and distribution of pores in low dielectric constant polymer films ${ }^{214}$, as well as the effects of exposure to plasma ${ }^{215}$ and high pressure cleaning liquids on them ${ }^{216}$.

To date, only a few positron spectroscopy studies of hydrophilic polymers and hydrogels have been reported. DBES and PALS studies were employed to investigate free volumes in a mixture and copolymers of poly(N-vinyl-pyrrolidone) and poly(ethylene glycol), which are widely used as drug delivery materials ${ }^{217}$; hydrogen bonded copolymers were found to contain larger free volume than covalently bonded copolymers. PALS studies were employed to study free volumes in $\gamma$ - and electron-beam crosslinked N-Isopropylacrylamide ${ }^{218}$; the free volumes and size distributions of the voids in the $\gamma$-and electron-beam crosslinked were found to be widely different. In a different study, the o-Ps lifetime, $\tau_{3}$, was measured for the homopolymers of NIsopropylacrylamide and acrylamide and several copolymers of the two monomers ${ }^{219}$. 
With increasing amounts of acrylamide in the copolymer, $\tau_{3}$ decreased, possibly due to increased polymer-polymer interactions, resulting in smaller free volume. Since the crosslink density in both homopolymers and copolymers was maintained at the same level by using the same amount of crosslinking agent, no correlation between crosslink density and free volume measured by PALS was possible.

\subsection{Experimental Section}

Free volume characteristics of plasma polymerized NIPAAm thin films deposited on silicon, and prepared under four different reactor conditions consisting of two different substrate temperatures and reactor pressures $\left(120^{\circ} \mathrm{C}, 133.3 \mathrm{~Pa} ; 120^{\circ} \mathrm{C}, 93.3 \mathrm{~Pa}\right.$; $175^{\circ} \mathrm{C}, 133.3 \mathrm{~Pa}$; and $175^{\circ} \mathrm{C}, 93.3 \mathrm{~Pa}$ ), were studied in Prof. Jean's group at University of Missouri-Kansas City (UMKC) using a slow positron beam. The design of the UMKC slow positron beam, which may be used to measure S parameters and positron lifetime, is shown in Figure 5.2

S parameters of plasma polymerized NIPAAm thin films were recorded at various depths by regulating the energy of the positrons $(0-30 \mathrm{keV})$ from the source. As mentioned earlier, the penetration depth of positrons scales as $\mathrm{E}^{1.6}$, and is given by the empirical relationship $^{204}$ :

$$
z=\left(\frac{400}{\rho}\right) E^{1.6}
$$

where, $z=$ penetration depth in $\AA, \rho$ is the density of the material in $\mathrm{g} / \mathrm{cm}^{3}$, and $E$ is the positron incident energy in $\mathrm{keV}$. 


\section{UMKC Slow Positron Beam (0 - 30keV)}

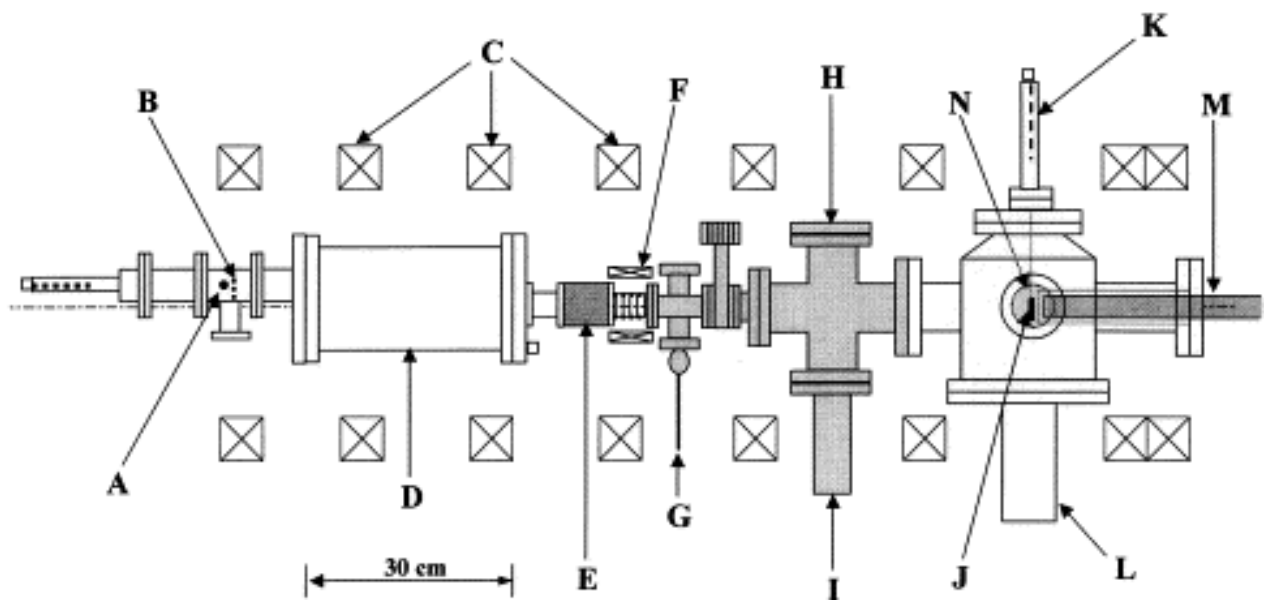

Figure 5.2 A schematic diagram of the slow positron beam at the University of MissouriKansas City. A: $50 \mathrm{mCi}^{22} \mathrm{Na}$ positron source, B: W-mesh moderator, C: magnetic field (75 G) coils, D: E X B filter, E: positron accelerator and electric insulator, F: correcting magnets, G: gas inlet, H: positron lifeline system for PAL, I: turbo molecular pump, J: samples, K: sample manipulator, L: cryo pump, M: Ge solid state detector, N: PAL detector $^{5}$

\subsection{Results and Discussion}

The S parameters of plasma polymerized NIPAAm thin films are shown as a function of positron incident energy $(\mathrm{keV})$ and calculated mean depth $(\mu \mathrm{m})$ in Figure 5.3 (a) \& (b). At very low positron incident energies, the S parameter is small due to back diffusion of the positrons. Positrons with higher incident energy, which are able to penetrate the plasma polymerized NIPAAm thin films, demonstrate higher S parameters. As the positron energy is increased further, the value of the $\mathrm{S}$ parameter begins to decrease since the positrons encounter the interfacial region of the hydrogel and substrate (silicon in this case), and subsequently the substrate itself at much higher energies. 

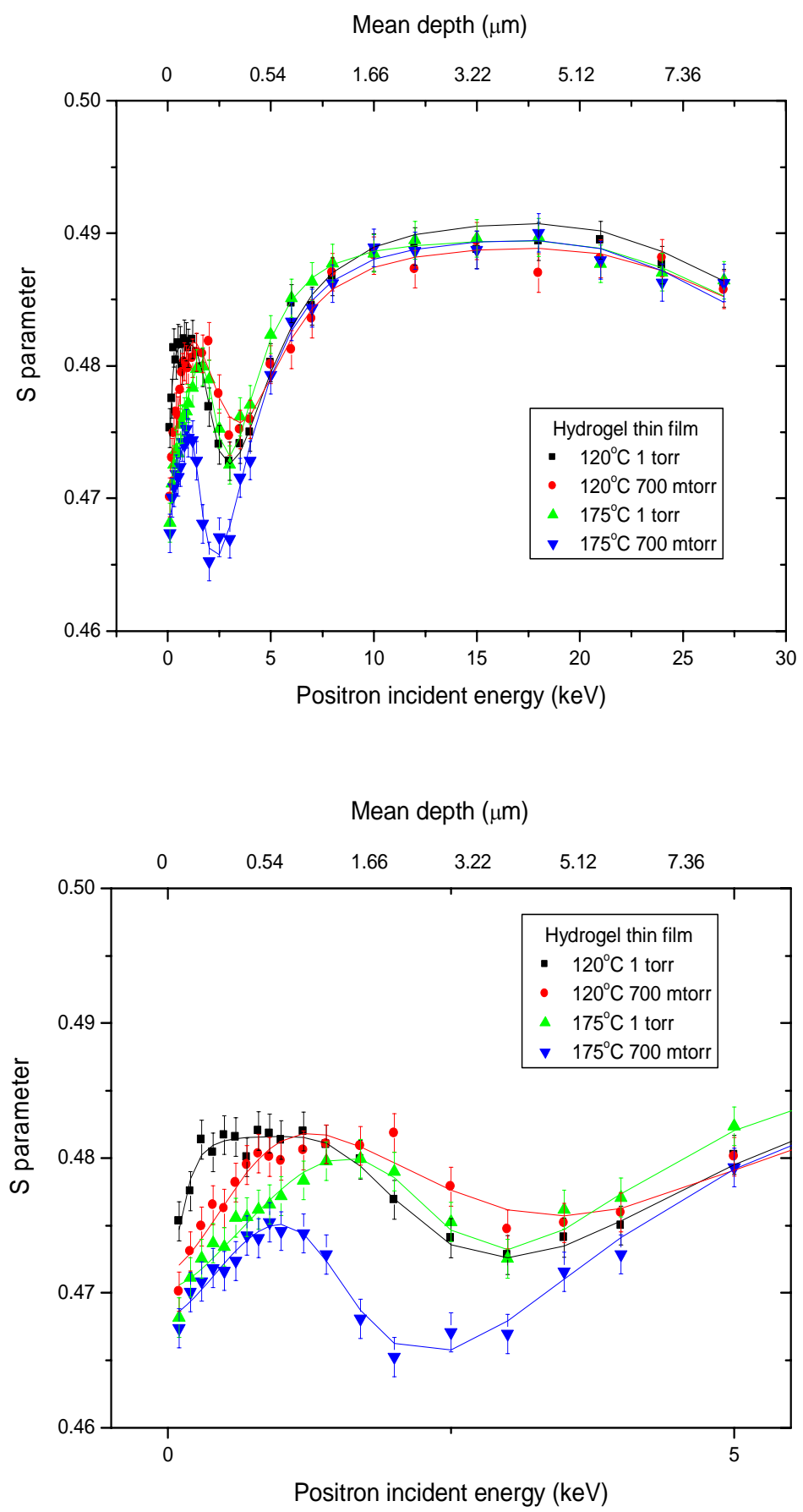

Figure 5.3 Variation of S parameter as a function of positron incident energy in plasma polymerized NIPAAm thin films prepared under different process conditions: (a) $0<$ positron incident energy $<30 \mathrm{keV}$ (top) (b) $0<$ positrons incident energy $<5 \mathrm{keV}$ (bottom) 
Table 5.1 Thickness and refractive index of plasma polymerized NIPAAm films from variable angle spectroscopic ellipsometry

\begin{tabular}{|c|c|c|}
\hline Process condition & Thickness $(\AA)$ & Refractive index at $600 \mathrm{~nm}$ \\
\hline $120^{\circ} \mathrm{C}, 133.3 \mathrm{~Pa}$ & $1505.7 \pm 3.1$ & 1.5259 \\
\hline $120^{\circ} \mathrm{C}, 93.3 \mathrm{~Pa}$ & $1828.7 \pm 1.2$ & 1.5305 \\
\hline $175^{\circ} \mathrm{C}, 133.3 \mathrm{~Pa}$ & $1778.5 \pm 0.8$ & 1.5207 \\
\hline $175^{\circ} \mathrm{C}, 93.3 \mathrm{~Pa}$ & $1272.4 \pm 1.5$ & 1.5614 \\
\hline
\end{tabular}

If the density is known, the thickness of the hydrogel film can be computed from the value of energy at full width half maximum (FWHM) of the S parameter peak by using Eq. $(5.1)^{206}$; greater FWHM refers to greater thickness, and vice-versa. Thickness and refractive index of plasma polymerized NIPAAm thin films measured using variable angle spectroscopic ellipsometry (VASE), before they were subjected to positron irradiation during DBES, are given in Table 5.1. From Figure 5.3 (b), it is clear that the trend in the values of FWHM follows the trend in thickness values measured for plasma polymerized NIPAAm films prepared under different process conditions (Table 5.1). Furthermore, for positron incident energies below $1 \mathrm{keV}$, which corresponds approximately to a sampling depth of $363 \AA$ if the hydrogel film is assumed to have a density of $1.1 \mathrm{~g} / \mathrm{cm}^{3}$, the $\mathrm{S}$ parameter values are the highest for NIPAAm films prepared at $120^{\circ} \mathrm{C}$ and $133.3 \mathrm{~Pa}$; the process conditions for NIPAAm in the decreasing order of $\mathrm{S}$ parameter values are $120^{\circ} \mathrm{C}, 93.3 \mathrm{~Pa}>175^{\circ} \mathrm{C}, 133.3 \mathrm{~Pa}>175^{\circ} \mathrm{C}, 93.3 \mathrm{~Pa}$. This trend in the S parameter values, and hence free volumes, agrees with the analyses in chapters 3 
and 4; films produced at higher substrate temperatures and lower pressures may be expected to possess greater crosslink density, and hence lower free volume (lower S parameter value), whereas films produced at lower substrate temperatures and higher pressures may be less crosslinked and hence, contain greater free volume (higher $\mathrm{S}$ parameter value). However, since the films studied using DBES are not of the same thickness, it is difficult to extend the preceding analysis to the full range of positron incident energies studied. Therefore, the variation in the S parameters may need to be fit to a multi-layer model consisting of three regions: near surface (region I), hydrogel film (region II), interface between hydrogel and silicon, and bulk silicon substrate (region III) ${ }^{206}$. Results from such analysis are shown in Table 5.2.

The S parameter values for the bulk, S3 layer are almost identical for plasma polymerized NIPAAm films prepared under all four processing conditions, perhaps because the S3 layer refers to the bulk silicon substrate and the interface between silicon and the hydrogel film. The S parameters for the second layer (S2), and the top layer (S1) are different for the different process conditions. At both substrate temperatures considered in this study, values of S2 are lower for films prepared at lower pressure, suggesting that the free volume is lower in films formed at lower pressures relative to higher pressures. The values of refractive index, which may be considered to be rough indicators of film density and crosslink density, and shown in Table 5.1 show a similar trend: plasma polymerized NIPAAm films formed at lower pressures have a higher refractive index relative to the films prepared at higher pressures. Values of S2 and refractive index are shown in Figure 5.4. On the other hand, at a given pressure, the films 
Table 5.2 Fitted parameters (from VEPFIT ${ }^{2}$ ) for DBES plots shown in Figure 5.1

\begin{tabular}{|c|c|c|c|c|c|c|c|c|}
\hline $\begin{array}{l}\text { Process } \\
\text { condition }\end{array}$ & $\begin{array}{c}\text { Top } \\
\text { Layer S1 }\end{array}$ & $\begin{array}{l}\text { Second } \\
\text { layer S2 }\end{array}$ & $\begin{array}{c}\text { Bulk } \\
\text { S3 }\end{array}$ & $\begin{array}{l}\text { Thickness } \\
\text { L1 (nm) }\end{array}$ & $\begin{array}{l}\text { Thickness } \\
\text { L2 (nm) }\end{array}$ & $\begin{array}{l}\text { Diffusion } \\
\text { Length D1 }\end{array}$ & $\begin{array}{l}\text { Diffusion } \\
\text { Length D2 }\end{array}$ & $\begin{array}{c}\text { Diffusion } \\
\text { Length D3 }\end{array}$ \\
\hline $133.3 \mathrm{~Pa}$ & 0.48165 & 0.44959 & 0.49122 & 111.43 & 282.54 & 17.99 & 87.27 & 224.72 \\
\hline $\begin{array}{l}120^{\circ} \mathrm{C}, \\
93.3 \mathrm{~Pa}\end{array}$ & 0.50237 & 0.43135 & 0.48924 & 65.26 & 683.26 & 49.20 & 38.12 & 95.30 \\
\hline $\begin{array}{c}175^{\circ} \mathrm{C} \\
133.3 \mathrm{~Pa}\end{array}$ & 0.49055 & 0.4511 & 0.48964 & 220.06 & 346.80 & 62.98 & 26.19 & 118.88 \\
\hline $\begin{array}{l}175^{\circ} \mathrm{C}, \\
93.3 \mathrm{~Pa}\end{array}$ & 0.4859 & 0.44391 & 0.48988 & 106.46 & 142.94 & 36.18 & 23.53 & 209.79 \\
\hline
\end{tabular}




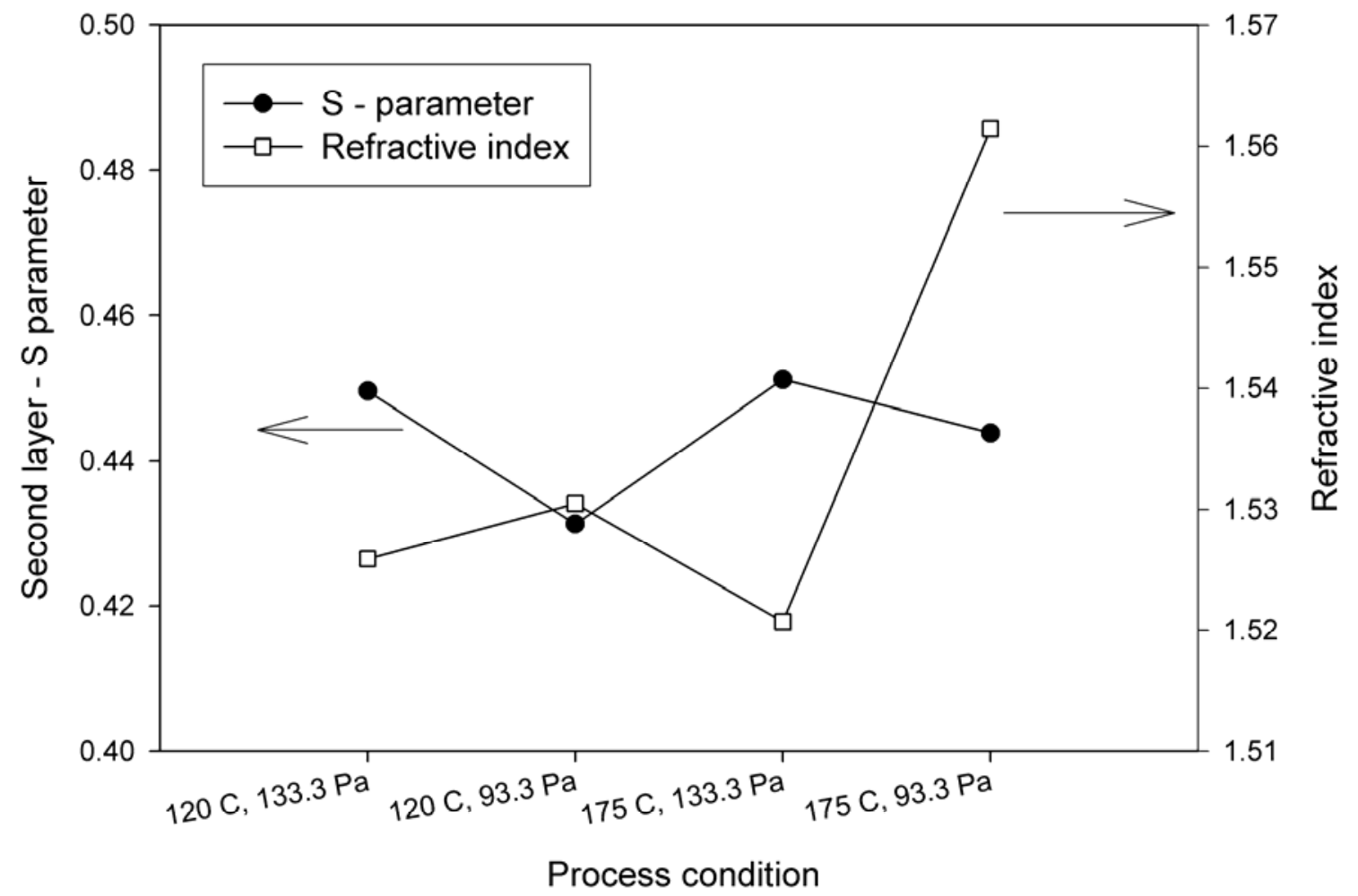

Figure 5.4 Values of S parameter of the second layer and refractive index of plasma polymerized NIPAAm films prepared under different reactor conditions.

formed at the higher temperature $\left(175^{\circ} \mathrm{C}\right)$ have a higher $\mathrm{S} 2$ value relative to the films formed at the lower temperature $\left(120^{\circ} \mathrm{C}\right)$, indicating that plasma polymerized NIPAAm films prepared at lower substrate temperature may possess greater free volume than hydrogel films films formed at higher substrate temperature. The correlation between S1 values and process conditions is not clear. From Table 5.2, plasma polymerized NIPAAm film prepared at a substrate temperature of $120^{\circ} \mathrm{C}$ and a pressure of $93.3 \mathrm{~Pa}$ has 
the highest value of S2, suggesting that this film may have the greatest free volume in the topmost layer; the reasons for this are not immediately obvious.

In Table 5.2, the sum of L1 and L2 (thickness values of layers 1 and 2), does not match the thickness values obtained through VASE. This is not surprising because in the VEPFIT simulation software, a constant density was assumed for plasma polymerized NIPAAm films prepared under all process conditions, to perform a 'zero-order' analysis first before assigning/fitting different densities to the hydrogel films. Additionally, the diffusion lengths D1, D2, and D3, which are expected to be inversely correlated with the S parameter and free volume ${ }^{210}$, do not show a clear correlation to the process conditions. It is plausible that a more rigorous simulation, which accounts for the different densities of hydrogel films prepared under different process conditions, may yield parameters that may show unambiguous correlations to the process conditions. Furthermore, a complete set of slow positron experiments involving measurement of positronium lifetimes may be required to determine size and depth profile of free volume.

\subsection{Conclusions}

In DBES experiments, $\mathrm{S}$ parameters, which are qualitative indicators of free volume, were recorded for plasma polymerized NIPAAm films prepared under different process conditions. S parameters were recorded for different depths in the hydrogel films by varying the positron incident energy. The trend in the FWHM of the S parameter depth profiles for hydrogel films prepared under different process conditions, matches the trend in the hydrogel film thickness measured independently using ellipsometry. 
Furthermore, for positron incident energy $<1 \mathrm{keV}$, which corresponds to a maximum sampling depth of $363 \AA$ for a hydrogel film density of $1.1 \mathrm{~g} / \mathrm{cm}^{3}$, the process conditions in decreasing order of the $\mathrm{S}$ parameter are: $120^{\circ} \mathrm{C}, 133.3 \mathrm{~Pa}>120^{\circ} \mathrm{C}, 93.3 \mathrm{~Pa}>175^{\circ} \mathrm{C}$, 133.3 $\mathrm{Pa}>175^{\circ} \mathrm{C}, 93.3 \mathrm{~Pa}$. This trend in the $\mathrm{S}$ parameter, and hence free volume, agrees with the analysis presented in chapters 3 and 4 , when the free volume is assumed to be inversely correlated with crosslink density ${ }^{213}$. Since the hydrogel films were not of identical thickness, trends in the $\mathrm{S}$ parameters for positron incident energy $>1 \mathrm{keV}$ are complex, and have to be analyzed by fitting the data to multilayer hydrogel structures. Preliminary simulations (using VEPFIT software) performed using a constant density for all hydrogel films yielded parameters that did not possess a clear correlation to the process conditions. It is expected that rigorous simulations that account for the different densities of the hydrogel films and a complete set of slow positron experiments involving measurement of positronium lifetimes will yield information on the microstructure of the hydrogel films that may be correlated to the process conditions. 


\section{CHAPTER 6}

\section{SUMMARY AND RECOMMENDATIONS}

\subsection{Summary}

Plasma polymerization is an attractive technique to prepare hydrogel thin films because randomly crosslinked thin films are deposited in a single step, and unlike conventional, solution polymerization methods, several chemical reagents such as initiators and crosslinking agents are not required. In this work, plasma polymerization was used to prepare hydrogel thin films based on N-Isopropylacrylamide (NIPAAm) in a capacitively coupled, parallel plate reactor. Physicochemical characteristics of the hydrogel thin films such as crosslink density and chemical bonding structure could be controlled by varying the substrate temperature and reactor pressure. From a parametric study based on substrate temperature and reactor operating pressure, it was found that hydrogel films formed at lower substrate temperatures and higher reactor pressure, showed greater reduction in thickness upon exposure to deionized water, relative to the hydrogel films formed at higher substrate temperatures and lower reactor pressure; the reduction in thickness is believed to occur due to dissolution of the uncrosslinked chains from inadequately crosslinked networks. FTIR studies revealed that the degree of hydrogen bonding in plasma polymerized NIPAAm films formed at higher substrate temperatures and lower reactor pressures was lower than in hydrogel films formed at lower substrate temperatures and higher reactor pressures. Furthermore, temperature dependent contact angle measurements confirmed the existence of a hydrophilic- 
hydrophobic transition, similar to a lower critical solution temperature (LCST) transition found in linear chains of NIPAAm.

Liquid water and moisture uptake characteristics of plasma polymerized NIPAAm films were studied between the temperatures of 20 and $40{ }^{\circ} \mathrm{C}$ using the quartz crystal microbalance with dissipation monitoring. In the temperature range studied, NIPAAm films showed continuous changes in both frequency $(\Delta \mathrm{f})$ and dissipation $(\Delta \mathrm{D})$, with higher $|\Delta \mathrm{f}|$ and $\Delta \mathrm{D}$ at higher temperatures, thus confirming the existence of a temperature dependent phase transition. In addition, in liquid water, NIPAAm films exhibited frequency dependent changes in frequency and dissipation, indicating viscoelastic behavior. In contrast, moisture uptake from the ambient (relative humidity $~ 40 \%$ ) did not reveal frequency dependent changes in $\Delta \mathrm{f}$ and $\Delta \mathrm{D}$, and therefore Sauerbrey formalism was applied to quantify the moisture uptake capacities of NIPAAm films. Intermolecular hydrogen bonding rather than the apparent crosslink density was found to control the moisture uptake capacity of NIPAAm films; NIPAAm films formed at lower substrate temperature and higher reactor pressure, with a lower apparent crosslink density, but with high degree of intermolecular hydrogen bonding exhibited lower moisture uptake capacities relative to NIPAAm films formed at higher substrate temperature and lower reactor pressure.

Doppler broadening of $\gamma$ rays emitted upon bombardment of hydrogel films by low energy positrons was analyzed to investigate free volumes of NIPAAm films prepared under different process conditions. The S-parameter, which is an indicator of 
free volume, was higher in NIPAAm films prepared at lower substrate temperature and higher reactor pressure relative to NIPAAm films prepared at higher substrate temperature and lower reactor pressure.

\subsection{Recommendations for future work}

- Characterization of mechanical properties: Determination of viscoelastic properties and intrinsic stresses in plasma polymerized NIPAAm films through nanoindentation and other direct methods are recommended. Quartz crystal microbalance studies in liquid water demonstrated that plasma polymerized NIPAAm films are viscoelastic (Chapter 4); the viscoelastic properties are believed to influence the signal from acoustic sensor employing NIPAAm films (Chapter 6). Knowledge of mechanical properties of NIPAAm films prepared under different reactor conditions, and exposed to different temperatures and levels of hydration, can give greater insight on crosslinking, chemical bonding structures, phase transition characteristics, and hence allow better design for various applications.

- Electrophoretic immobilization procedure: In order to evaluate the efficiency of an electrophoretic procedure to incorporate biomolecules in the hydrogel matrix, a quantitative measure of the amount of biomolecules incorporated in the hydrogel matrix is required. Subsequently, based on the level of biomolecule incorporation, the electrophoretic immobilization procedure may be tailored to achieve an optimum level of incorporation. Two important process variables in 
the electrophoretic procedure that may be varied are applied voltage and the duration of electrophoretic immobilization. At higher voltages, electrolysis of the buffer solution containing the biomolecules is likely; gas evolution during the electrolysis can damage the hydrogel matrix due to the mechanical forces imposed on it by the bubbles. Similarly, the time of electrophoretic incorporation is also important: longer times can result in damage to the hydrogel matrix, and possibly denaturation of the biomolecules (or proteins).

- Biomolecule patterning: If the electrophoretic immobilization procedure is performed using micro(nano)electrodes to introduce spatial resolution, in an approach similar to dip-pen nanolithography ${ }^{184}$, electrophoretic immobilization procedure may be used to prepare arrays/patterns of proteins and nucleic acids by drifting charged biomolecules from small volumes of biomolecule containing media on hydrogel films formed from plasma polymerization. Since the electrophoretic procedure may be adapted for incorporation of biomolecules on a large scale, mass production of protein and biomolecule containing arrays may be possible.

- Biosensor development: In this work, antibody containing hydrogel films were employed in gravimetric sensors based on acoustic resonator devices. However, biomolecule containing hydrogel films prepared using the electrophoretic procedure may also be used in sensors operating on electrical, electrochemical, and optical transduction principles. 


\title{
APPENDIX A \\ ELECTROPHORETIC IMMOBILIZATION AND SENSOR DEVELOPMENT
}

\begin{abstract}
A.1 Introduction
Proteins such as enzymes and antibodies are capable of highly specific biomolecular interactions, and hence, form the basis of chemical / biomolecular recognition layers in a variety of sensors. However, to be useful in the sensor format, proteins need to be immobilized on the surface or at the interface of transducers. Chemical / biomolecule-sensitive layers for application in sensors may be prepared by either immobilizing proteins at the surface of a transducer in a two-dimensional approach, or by incorporating them in the bulk of porous support media such as hydrogels (three-dimensional approach). In the two-dimensional approach, biomolecules may be conjugated to various surfaces through homo- and hetero-functional crosslinking agents, or covalent bonding of specific groups in the proteins (acids, amines) to complementary groups on the surface using labor intensive methods that require expensive reagents ${ }^{118}$. Additionally, proteins may be adsorbed on surfaces containing ion-exchange or other charged groups through electrostatic adsorption forces ${ }^{173}$. In the three-dimensional approach, thin films may be formed from bulk gels containing proteins such as enzymes and antibodies such that the resulting film encapsulates the protein ${ }^{173,}$ 174. In a slightly different approach, a thin film may be formed in the presence of a
\end{abstract}


protein such that the growing film encapsulates the protein; for example, antibodies suspended in the precursor solution used for electropolymerization may be trapped within electropolymerized films, which may subsequently be used as immunosensing layers ${ }^{175}$.

Hydrogels are water swollen polymeric networks that are ideal for the creation of biomolecule-polymer composites due to their many potential applications in the fields of medicine, agriculture and biotechnology ${ }^{129}$, increased capacity for immobilization of proteins compared to two-dimensional surfaces, and the stable microenvironment they can provide with adequate hydration to ensure support for antigen or ligand binding ${ }^{176,}$ 177. Biomolecule containing hydrogel thin films are attractive biosensing platforms and ideal candidates for protein microarrays / biochips ${ }^{178-181}$, and microfluidic biosystems ${ }^{182}$, 183.

A common technique to prepare protein containing hydrogel thin films involves incorporating the protein in bulk hydrogel, followed by the use of a thin film formation procedure to coat a transducer. However, since thin film preparation through techniques such as spin or dip casting from complex, bulk materials such as hydrogels containing proteins, does not produce uniform, homogeneous films of low thicknesses ${ }^{101}$, the approach is not suitable for use in high-sensitivity sensors. A novel, electrophoretic procedure for incorporation of antibodies and other charged biomolecules from buffer solutions into hydrogel thin films produced using a well-controlled and reproducible RF plasma polymerization process, has been conceived; results from preliminary experiments are presented. The first step in the electrophoretic immobilization process 
consists of suspending antibody molecules in a buffer solution, which has a $\mathrm{pH}$ that is different from the $\mathrm{pI}$ of the antibody. Due to the difference in the values of $\mathrm{pI}$ of the antibody and the $\mathrm{pH}$ of the buffer, the antibody molecules are charged, hence, can be drifted into a hydrogel matrix under the influence of an electric field. The advantages of the electrophoretic incorporation procedure are as follows:

- Orientation-controlled immobilization of antibodies - Antibody molecules are charged when they are in a buffer solution with a $\mathrm{pH}$ different from their pI; when the charged antibodies are drifted into the hydrogel thin films under the influence of an electric field, the orientation is controlled.

- High density of recognition sites can be obtained by drifting charged antibodies into the bulk of the hydrogel thin films relative to immobilization on the surface.

- The microenvironment of three-dimensional hydrogel network films simulates a solution phase system in contrast to the solid-liquid interface presented by biomolecules attached to a surface.

- Eliminates the need for expensive reagents to conjugate proteins to surfaces.

- The electrophoretic incorporation procedure may be used to drift various charged biomolecules into hydrogel films using a scanning probe microscopy technique to obtain patterns for micro/nano-arrays in an implementation similar to dip-pen nanolithography ${ }^{184}$.

To the author's knowledge, an electrophoretic procedure for post-deposition immobilization of biomolecules in hydrogel thin films to prepare sensing layers has not 
been reported. Other electrochemical approaches to preparing biomolecule containing hydrogel / polymer films involve entrapment of biomolecules during electropolymerization ${ }^{175}$, and $\mathrm{pH}$-shift induced deposition of polymer containing biomolecules ${ }^{185}$, through a mechanism similar to electrocoating employed in the automobile industry. Furthermore, other types of electrophoretic/iontophoretic processes to prepare thin films and coatings through electrophoretic deposition, and infiltration of porous matrices with ceramic particles ${ }^{186}$, iontophoretic drug delivery ${ }^{187}$, and loading bulk hydrogels with protein drugs ${ }^{188}$ have also been reported previously.

In this work, label-free, immunosensors based on acoustic devices such as surface acoustic wave resonators (SAW) and quartz crystal microbalances (QCM) have been developed by using antibody containing hydrogel films, prepared through electrophoretic incorporation, as the sensing layers. Acoustic wave devices rely on the propagation of bulk and surface acoustic waves in piezoelectric materials such as quartz. Metal electrodes/transducers are used to excite ultrasonic waves in the solids; the characteristics of the acoustic waves such as resonant frequency and type are determined by the crystal orientation, thickness of the material, and the geometric shape of the electrodes/transducers. QCMs are made of thin discs of AT-cut quartz, and possess metal electrodes on either side to excite thickness shear mode (TSM) waves through the application of an oscillating electric field. The excited transverse waves propagate through the bulk of the device, and cause particle displacement parallel to the sensor surface. Since the thickness of the sensor determines the resonance frequency, and the overtones, the range in resonance frequencies that can be realized is limited by the 
thickness of the quartz crystal that can be achieved in practice. Higher resonance frequencies are achieved by thinner quartz discs and vice-versa; the general range of resonance frequencies is $5-20 \mathrm{MHz}$. SAW devices rely on the propagation of Rayleigh waves on the surface, causing the particles on the surface to move elliptically, thus causing a wave consisting of both shear and compressional components. A common form of SAW devices is the 'delay line' configuration with input and output metal transducers consisting of interdigitated electrodes (IDT) on thick ST-cut quartz plates. Unlike the QCM, which is a one-port device, the delay line SAW device is a two-port device. Resonance frequencies of SAW devices range from 30 to $500 \mathrm{MHz}$, and are determined by the finger spacing of the IDT.

Sensors based on acoustic wave resonators are mostly operated as gravimetric sensors, and rely on the change in the characteristics of the acoustic wave propagation, such as velocity, and resonance frequency, in response to changes in the mass loading on the sensor surface to detect analytes. Generally, a correlation based on Sauerbrey's relationship, which relates the measured change in resonance frequency to mass changes through a constant specific to the acoustic resonator ${ }^{146}$, is invoked to calculate the change in mass loading ${ }^{189}$. To capture only the analyte of interest, chemical or biomolecule recognition layers are deposited on the surface of acoustic resonators. In the present case, antibodies specific to a particular analyte immobilized in hydrogel layers deposited on SAW devices recognize the analyte through highly specific antigen-antibody interactions. Mass sensitivity, which may be defined as the ratio of the relative change in an acoustic wave propagation parameter such as resonance frequency to the change in mass loading, 
is dependent on the fundamental resonance frequency, and hence, is higher in SAW sensors than QCM sensors. The principles, operation and development of sensors based on acoustic devices such as SAW and QCM are described in greater detail in review articles ${ }^{149,190}$.

\section{A.2 Experimental Section}

Plasma polymerized thin films of N-Isopropylacrylamide (NIPAAm) were deposited on custom fabricated surface acoustic wave (SAW) devices in a $13.56 \mathrm{MHz}$, parallel plate reactor at a bottom electrode temperature of $175^{\circ} \mathrm{C}$ and a reactor pressure of 133.3 Pa. Details of the reactor and properties of the plasma polymerized NIPAAm films have been discussed in Chapters 3 and 4.

Preliminary experiments were conducted to attempt to immobilize antibodies in plasma polymerized NIPAAm films using an electrophoretic transfer technique from a $4 \%$ solution of mouse monoclonal anti-FITC in 1X-TAE (Tris acetate EDTA) buffer; both chemicals were purchased from Sigma-Aldrich Corp., St. Louis, USA. When diluted in 1X-TAE buffer ( $\mathrm{pH} \sim 8.3$ at $25^{\circ} \mathrm{C}$ ), anti-FITC takes on a negative charge, and hence will migrate towards the positive electrode in an electrophoretic apparatus. To immobilize anti-FITC, hydrogel coated SAW devices were immersed in a buffer solution containing anti-FITC. Subsequently, the Aluminum bonding pads of the SAW device underlying the NIPAAm film were positively biased with respect to the buffer (electrolyte) for 10 min. A schematic of the experimental apparatus is shown in Figure 
5.1. Electrical contact was achieved through micro-DC probes in a probe station; the experiment was continuously monitored through the microscope.

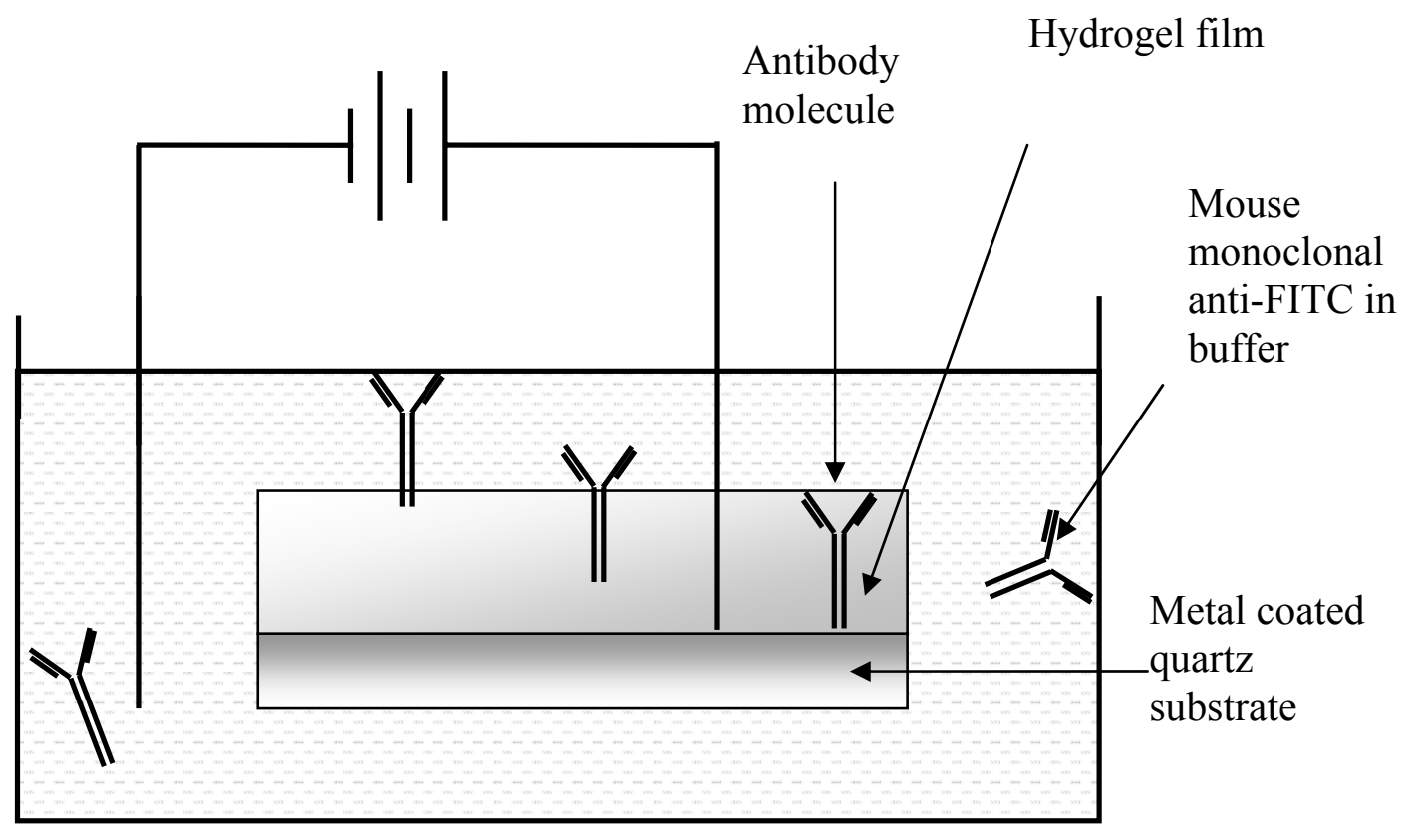

Figure A.1 Schematic of the electrophoretic immobilization set up (not drawn to scale).

A fluorescent immunoassay protocol ${ }^{191}$ was used to confirm the incorporation of anti-FITC in the hydrogel film. After the electrophoretic transfer process, the hydrogel films were exposed to a fluorescent analyte, Uranine (Fluka Chemika) vapor, washed with the buffer solution to remove the unbound antigen, and viewed using a Zeiss LSM150 confocal laser scanning microscope (CLSM). Any fluorescence detected in the hydrogel films may be attributed to the antibody-fluorescent antigen complex, and hence provided a confirmation of the incorporation of antibodies. In addition, control samples consisting of NIPAAm film on a SAW device not subjected to the electrophoretic incorporation procedure, and a blank SAW device, were also studied using the fluorescent immunoassay protocol. 
Frequency response of the SAW devices was recorded using a network analyzer. Specifically, $\mathrm{S}_{21}$ parameters of the SAW devices were recorded as a function of frequency, close to the resonant frequency of the SAW device at approximately 250 MHz. In order to determine the change in frequency response in response to antigenantibody binding, $\mathrm{S}_{21}$ parameters of the SAW devices were measured immediately after fabrication of the devices, after deposition of plasma polymerized NIPAAm on the SAW devices, after electrophoretic incorporation of anti-FITC, and finally after exposure to the antigen. Antigen vapor was presented to the SAW devices by bubbling $\mathrm{N}_{2}$ through a uranine solution $(90 \mu \mathrm{g} / \mathrm{ml})$ at the rate of $0.3 \mathrm{SLPM}$ (standard liters per minute) for $20 \mathrm{~s}$, followed by an air purge (1.85 SLPM) for $20 \mathrm{~s}$ to remove any antigen not bound to the antibody. Dr. Sang-Hun Lee of the Microelectronic Acoustics laboratory in the School of Electrical \& Computer Engineering, Georgia Institute of Technology, fabricated and measured the frequency response of the SAW devices, and assisted in the development of sensors through the electrophoretic immobilization procedure.

\section{A.3 Results And Discussion}

Initially, vapor phase SAW sensors for the detection of uranine vapor were developed by attempting to electrophoretically incorporate anti-FITC in NIPAAm films at $5 \mathrm{~V}$ and $1 \mathrm{~V}$; electrophoretic incorporation of anti-FITC was performed in the manner described previously. Frequency response measurements of the SAW devices were made immediately after NIPAAm deposition, after anti-FITC incorporation, and after antigen exposure. Figure 5.2 (a) and (b) show the frequency response plots of SAW devices 
containing anti-FITC incorporated at $5 \mathrm{~V}$ and $1 \mathrm{~V}$ respectively, whereas Figure 5.2 (c) shows the frequency response of the control sample, which was not subjected to the electrophoretic incorporation, and hence did not contain any anti-FITC. Immediately after anti-FITC incorporation at $5 \mathrm{~V}$ and $1 \mathrm{~V}$, an upshift of at least $2 \mathrm{MHz}$ was observed in the resonance frequency. Subsequent exposure to uranine vapor caused smaller increases $(0.15 \mathrm{MHz}$ in the case of $5 \mathrm{~V}$, and $0.05 \mathrm{MHz}$ in the case of $1 \mathrm{~V})$ in the resonance frequency of the SAW devices, in addition to increases in insertion loss.

Any change in the frequency response, specifically, in resonance frequency and insertion loss, may be due to a change in the interaction of the surface acoustic wave with the NIPAAm layer containing anti-FITC, which is capable of binding uranine molecules in the vapor phase. In an implementation similar to the one described above, vapor phase sensors were developed by coupling anti-FITC to gold coated SAW devices through protein A crosslinker; exposure to uranine vapor resulted in a negative shift in the resonance frequency ${ }^{191}$. The Sauerbrey relationship ${ }^{146}$ according to which, increased mass loading of an acoustic resonator is linearly proportional to the decrease in the resonance frequency, was invoked to attribute the negative shift in the resonance frequency to mass loading from uranine molecules that bind anti-FITC on the SAW device. However, in the present case, due to increased mass loading on the SAW devices after anti-FITC incorporation and antigen exposure, the resonant frequency has shown a positive shift, suggesting that the Sauerbrey type of analysis may not be applicable. 

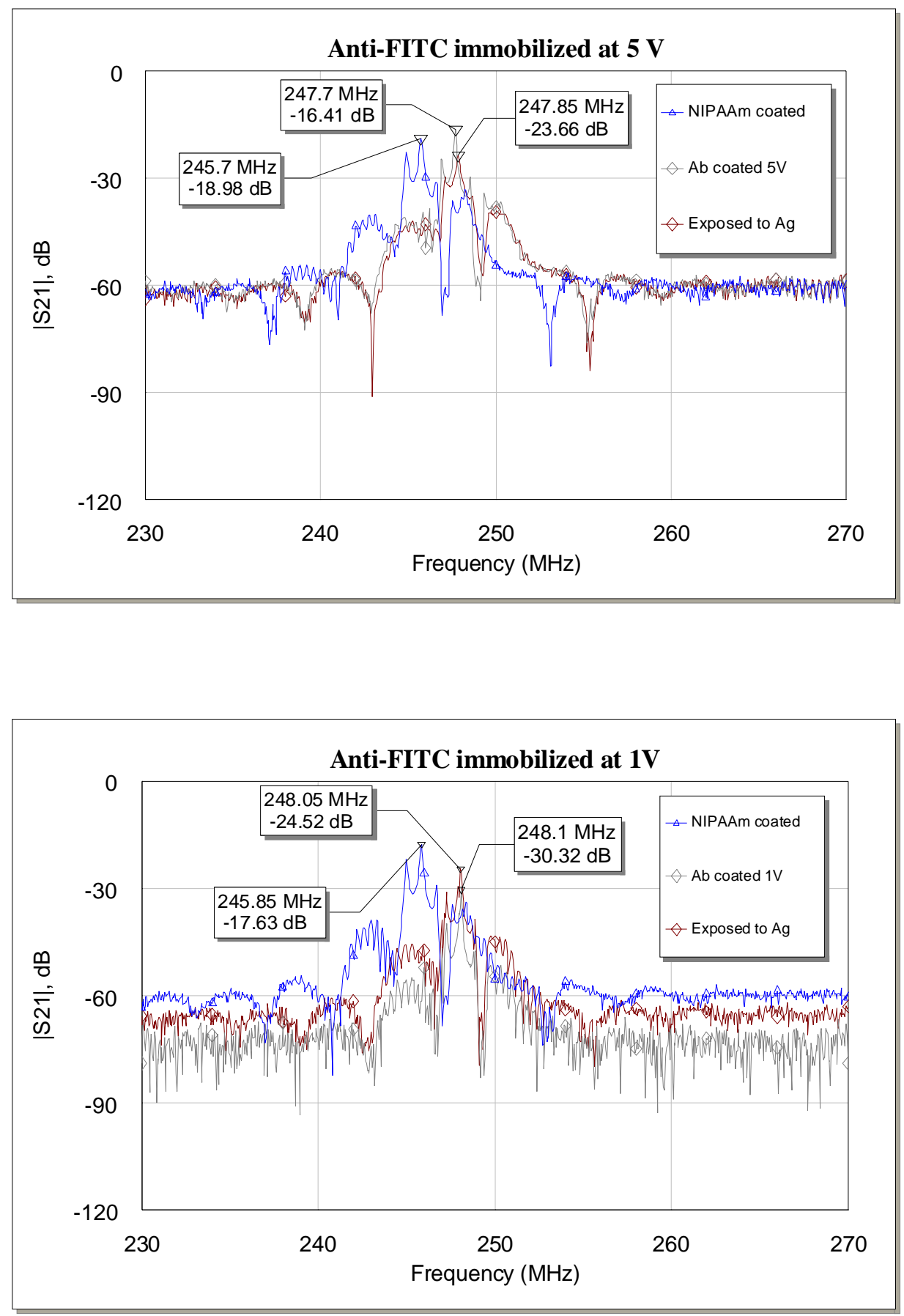

Figure A.2. Frequency response of SAW devices after NIPAAm deposition (---), after anti-FITC immobilization (---), and after exposure to uranine vapor (---); (a) Electrophoretic immobilization at $5 \mathrm{~V}$ (top); (b) Electrophoretic immobilization at $1 \mathrm{~V}$ (bottom) 


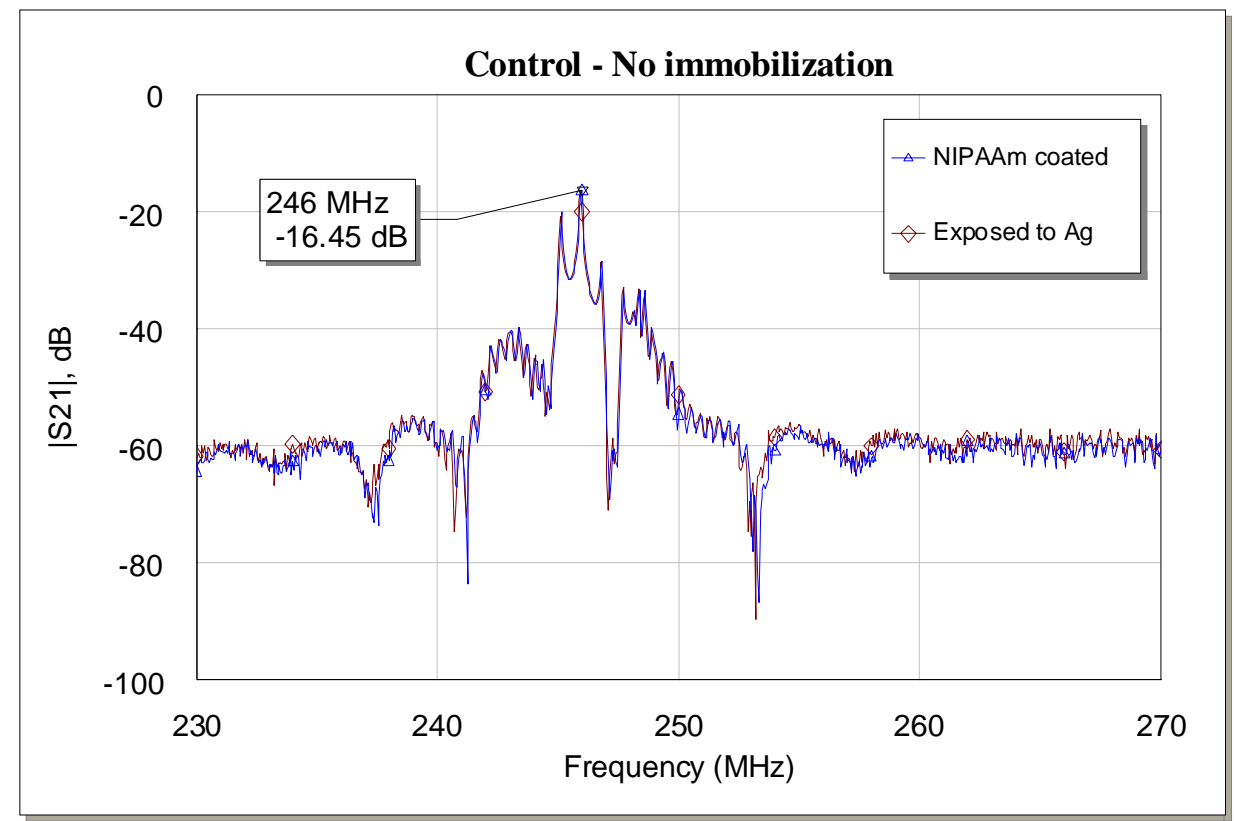

Figure A.2 continued Frequency response of SAW devices after NIPAAm deposition (--), and after exposure to uranine vapor (---); (c) No anti-FITC immobilization

While the exact reasons for the positive shift in resonance frequency are not immediately obvious, increased insertion loss upon exposure to uranine vapor suggest that antigenantibody binding may have occurred.

Indeed, several investigators have reported deviations from the Sauerbrey type of response in acoustic resonator based sensors. In one such study, anti-FITC was coupled to commercially available, $10 \mathrm{MHz}$ QCM through a self assembled monolayer; exposure of the anti-FITC containing QCM to an uranine solution resulted in a positive shift in the resonance frequency. The control sample, which was a QCM coated with antibodies not specific to uranine, did not show a change in the resonance frequency upon exposure to uranine solution $^{192}$. In a different study, frequency was found to increase when 
immunoglobulin $\mathrm{G}(\mathrm{IgG})$ was bound to antigen immobilized on the surface of a $\mathrm{QCM}^{193}$; the result was attributed to an alteration of the microviscosity at the interface upon binding with the antibody. In a similar study, despite an initial reduction in the frequency when cells were adsorbed on the surface of a QCM, after approximately 80 minutes, cell spreading caused an increase in the frequency, beyond the initial value recorded before exposure to the cells ${ }^{194}$. Based on the transient decay time constant and the oscillation amplitude maximum, the cells were found to have increased in stiffness, thus causing an increase in the frequency. Since phase contrast and fluorescence microscopy confirmed that the cells were adsorbed on the surface, frequency increase due to cell desorption was ruled out.

Recently, a time dependent perturbation analysis of QCM sensors showed that increased stiffness of the sensing layer can result in positive shifts in the resonance frequency, despite increased mass loading ${ }^{195}$. The derived relationship showed that mass loading causes negative shifts in the resonance frequency, in accordance with the Sauerbrey relationship, whereas increased stiffness of the overlayer causes positive shifts in the resonance frequency. Since mass loading and stiffness changes in the sensing layer were found to have opposite effects on the resonance frequency, based on the frequency shifts observed in QCM sensors, it may be possible to resolve the dominant mechanism. In fact, several other investigators have also shown that the frequency response of acoustic resonators is determined by not only the effect of mass loading, but also by the (visco)elastic properties of the overlayer ${ }^{196-199}$. Consequently, acoustic resonators may operate as gravimetric sensors only under specific conditions when the overlayer is rigid, 
acoustically thin, and analyte sorption does not cause a change in the (visco)elastic properties of the overlayer. The non-gravimetric response of acoustic resonators has proven useful in the study of shear modulus of thin films ${ }^{199}$, phase transitions ${ }^{200-202}$ and crosslinking $^{203}$.

An additional issue pertaining to the initial set of experiments concerns the electrophoretic immobilization procedure; at voltages of $1 \mathrm{~V}$ and higher, significant gas evolution was observed from electrolysis of the aqueous buffer in most experiments, which damaged the NIPAAm and aluminum films on the SAW device, and caused a loss in electrical connection to the aluminum bonding pads. To prevent electrolysis of the buffer and the resulting damage to the NIPAAm and aluminum films, a lower voltage $(0.5 \mathrm{~V})$ was used to incorporate anti-FITC in NIPAAm films coated on SAW devices. Since the electric field driving the antibodies into the NIPAAm film was lower at $0.5 \mathrm{~V}$ than at higher voltages, in addition to performing the electrophoretic incorporation for 10 minutes, as in the case of higher voltages, separate experiments were conducted for 15 minutes. Frequency response plots of the SAW devices after NIPAAm deposition, after anti-FITC incorporation, and after uranine exposure are shown in Figure 5.3.

While incorporation of anti-FITC resulted in a small negative shift of the resonance frequency, suggesting increased mass loading, there was no change in the resonance frequency after uranine exposure. Therefore, based on the frequency response data, it is not clear whether antibody-antigen binding occurred on the SAW devices since the frequency and insertion loss associated with the $S_{21}$ peak did not change significantly 
before and after exposure to the antigen. Furthermore, control samples consisting of a NIPAAm coated SAW device, not subjected to the electrophoretic incorporation procedure, and a blank SAW device, showed small negative shifts in the resonance frequency upon exposure to uranine vapor, suggesting small amounts of mass loading, perhaps due to non-specific binding.

Examination of the SAW devices using CLSM (Figure 5.4) revealed several fluorescent spots in the region of interdigitated electrodes on the device subjected to the electrophoretic immobilization procedure at $0.5 \mathrm{~V}$ for 15 minutes. However, the SAW device subjected to the electrophoretic procedure at $0.5 \mathrm{~V}$ for 10 minutes did not demonstrate adequate fluorescence, suggesting that at the lower voltage of $0.5 \mathrm{~V}$, at least 15 minutes may be required for incorporation of anti-FITC in the matrix of NIPAAm films. Additionally, control samples consisting of a NIPAAm film on the SAW device, and a blank SAW device did not exhibit adequate fluorescence upon exposure to uranine vapor, suggesting that the non-specific adsorption of uranine vapor was minimal. Since fluorescence in the NIPAAm films must arise from the bound complex of anti-FITC and uranine, the fluorescent immunoassay confirms that the electrophoretic procedure at 0.5 V for 15 min incorporated anti-FITC in the hydrogel films. 

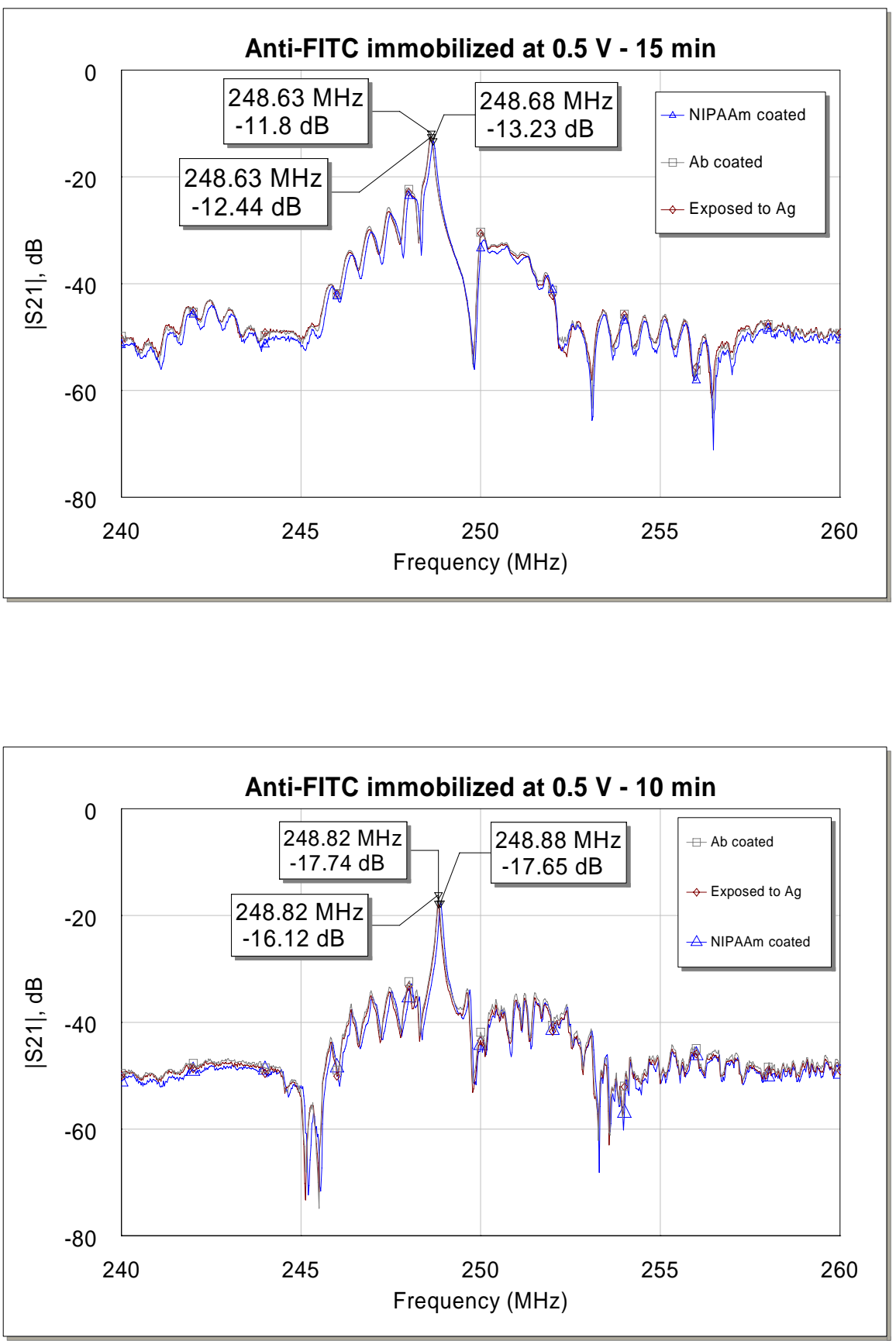

Figure A.3. Frequency response of SAW devices after NIPAAm deposition (---), after anti-FITC immobilization (---), and after exposure to uranine vapor (---); (a) Electrophoretic immobilization at $0.5 \mathrm{~V}$ for $15 \mathrm{~min}$ (top); (b) Electrophoretic immobilization at $0.5 \mathrm{~V}$ for $10 \mathrm{~min}$ (bottom) 

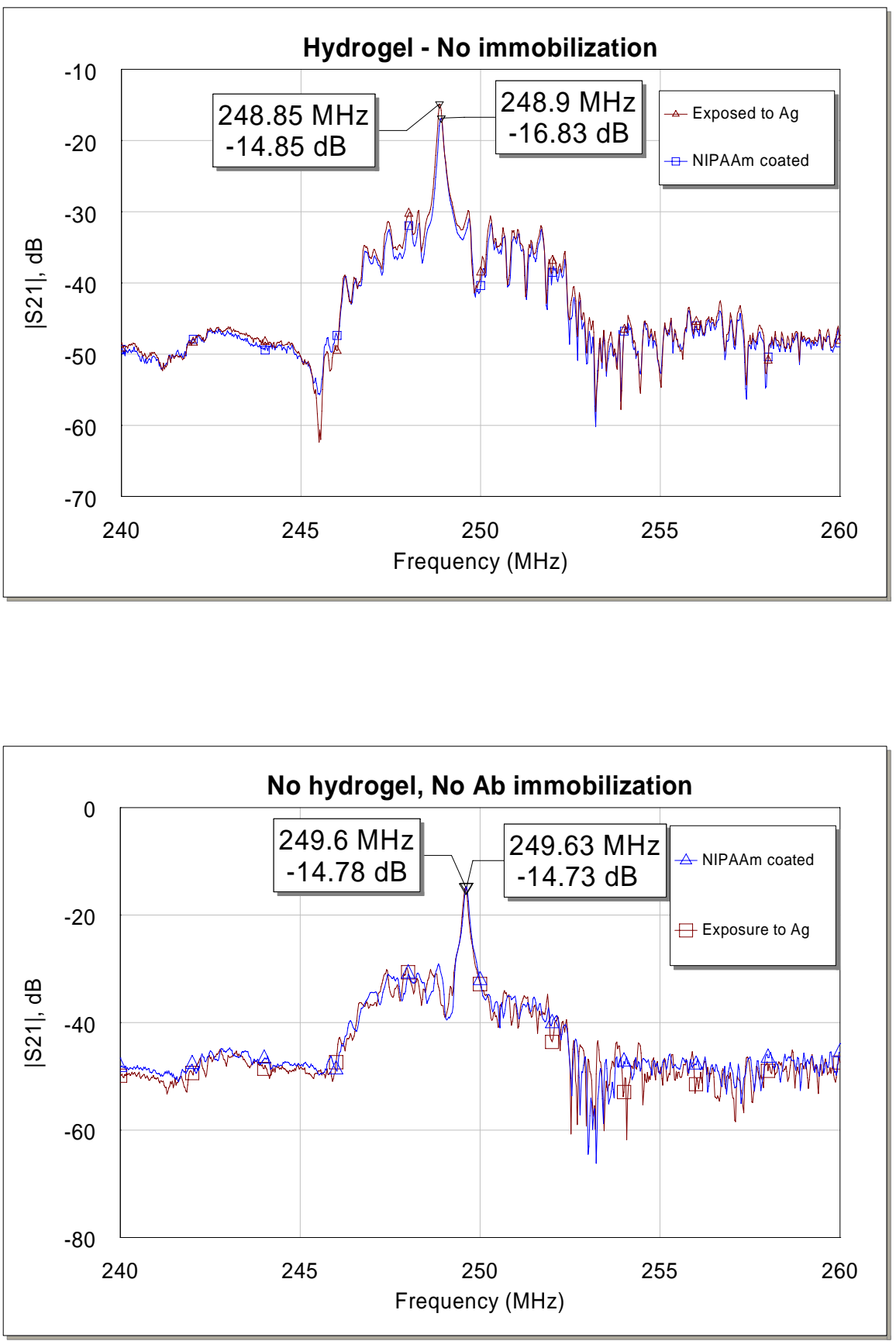

Figure A.3 continued Frequency response of SAW devices after NIPAAm deposition (--), and after exposure to uranine vapor (---); (c) NIPAAm film - no electrophoretic immobilization (top); (d) Blank SAW device - No NIPAAm film, no electrophoretic immobilization (bottom) 


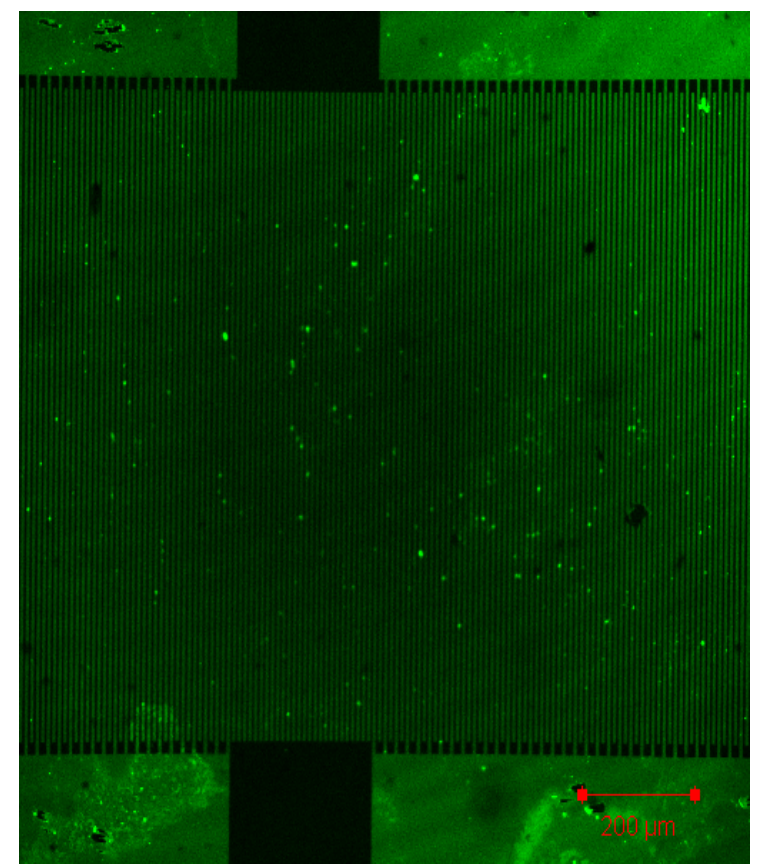

a

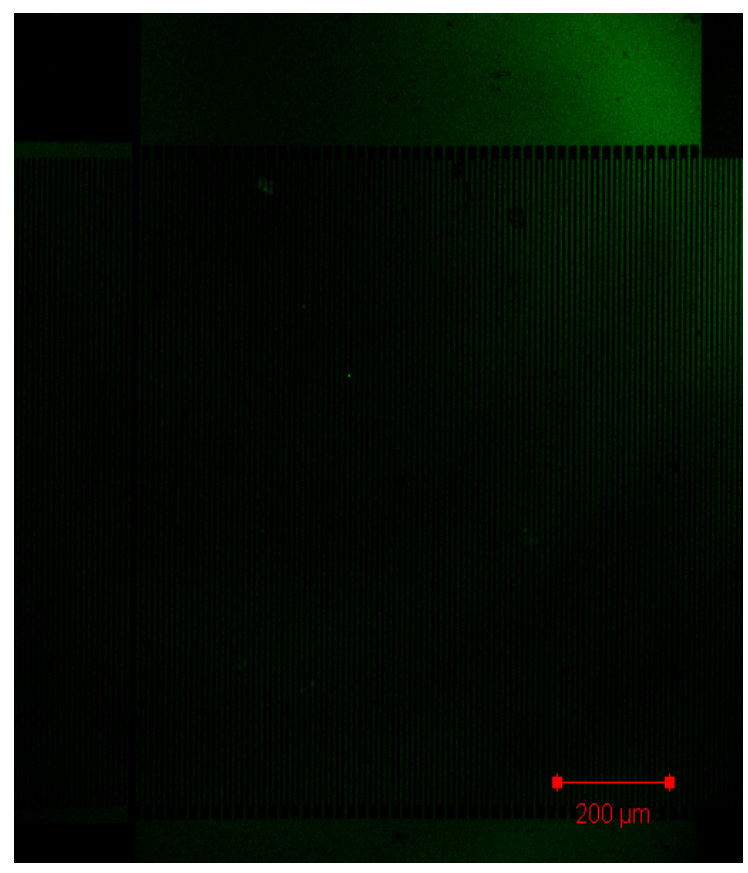

c

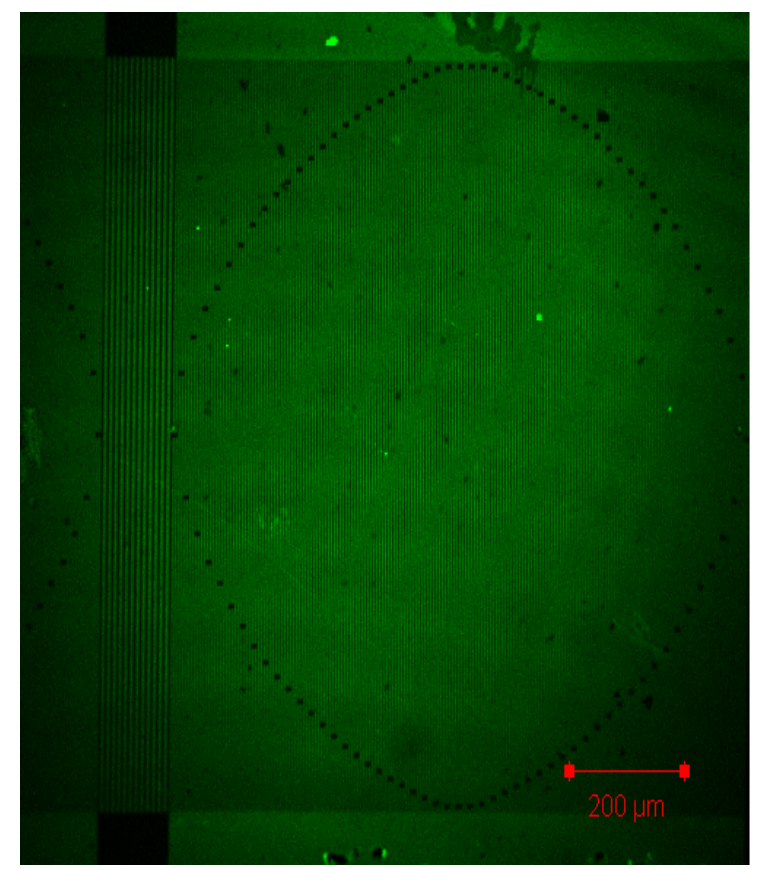

$\mathrm{b}$

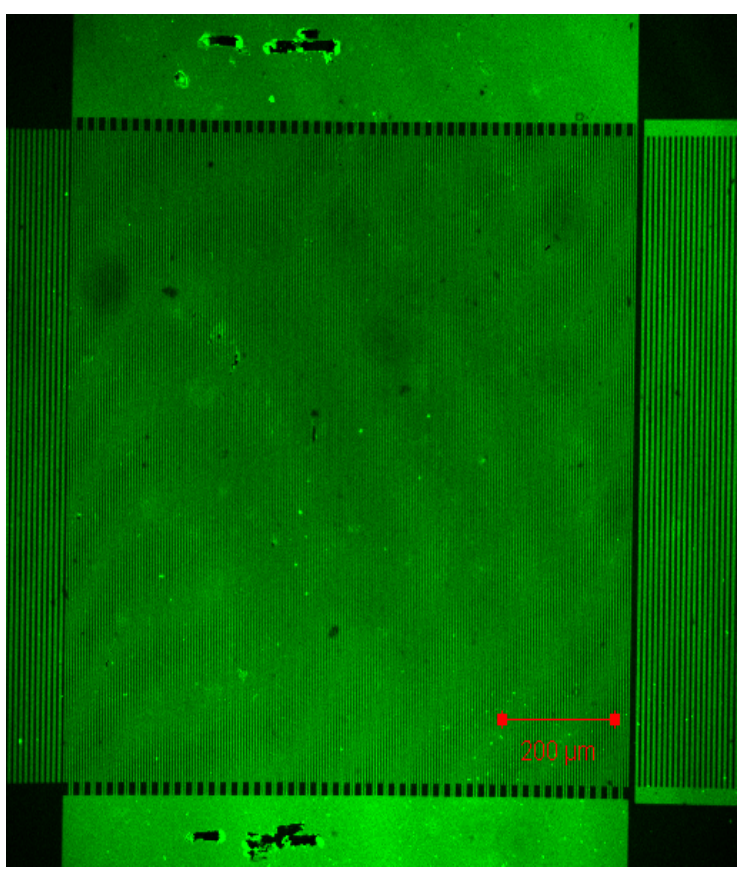

d

Figure A.4 CLSM images: (a) Anti-FITC containing NIPAAm film (0.5 V for $15 \mathrm{~min})$; (b) Anti-FITC containing NIPAAm film (0.5 V for $10 \mathrm{~min})$; (c) Blank SAW device - no hydrogel film; (d) Hydrogel coated SAW device - no anti-FITC. 
The reason for the lack of a definitive signature in the frequency response after uranine exposure in the device subjected to the electrophoretic incorporation procedure for 15 min despite evidence from CLSM suggesting that the immobilization was successful is not immediately obvious. One possible reason could be the high detection limit of $25 \mathrm{kHz}$ imposed by the network analyzer. In fact, in previous reports on SAW sensors developed to detect uranine vapor, the baseline shift in the frequency of the SAW

devices was less than the detection limit utilized in the above experiments ${ }^{191,195}$. In future experiments, frequency response may need to be studied on-line with measurement equipment capable of realizing lower detection limits. In addition, the immobilization density of antibody molecules may need to be improved by conducting the electrophoretic immobilization procedure from concentrated antibody solutions, or by increasing the time of immobilization.

\section{A.4 Conclusions}

A novel, electrophoretic procedure was conceived to incorporate biomolecules such as antibodies in plasma deposited hydrogel thin films. In preliminary experiments, attempts were made to incorporate anti-FITC molecules in plasma polymerized NIPAAm films deposited on $250 \mathrm{MHz}$ SAW sensors to detect uranine in the vapor phase. While a definite acoustic signature, characteristic of the antigen-antibody binding event was not observed, CLSM images from a fluorescent immunoassay seem to confirm the existence of anti-FITC in the hydrogel film. Future experiments may focus on achieving higher densities of antibody molecules in NIPAAm films, and improving the detection limit of the SAW sensors. 


\section{APPENDIX B}

\section{STABILITY STUDIES}

To use plasma polymerized NIPAAm thin films in sensors and other types of devices, they must be stable in a variety of aqueous environments. Stability depends on the physicochemical structure of the films, which suggests that films obtained under different processing conditions can show differing levels of stability. For the purposes of experimental evaluation, NIPAAm films prepared under four different conditions of substrate temperature and operating pressure $\left(120^{\circ} \mathrm{C} \& 133.3 \mathrm{~Pa}, 120{ }^{\circ} \mathrm{C} \& 93.3 \mathrm{~Pa}, 175\right.$ ${ }^{\circ} \mathrm{C} \& 133.3 \mathrm{~Pa}$ and $175^{\circ} \mathrm{C} \& 93.3 \mathrm{~Pa}$ ) were exposed to deionized (DI) water, phosphate buffered saline (PBS) solution $(\mathrm{pH} \sim 7.3)$, and $1 \mathrm{X}$ Trisacetate EDTA (TAE) buffer solution $(\mathrm{pH} \sim 8.3$ ) for a period of three weeks; changes in the chemical bonding structure and thickness as a result of the prolonged exposure to DI water and buffer solutions were investigated using FTIR spectroscopy (Bruker IFS 66 v/s spectrometer) and variable angle spectroscopic ellipsometry (J. A. Woollam M-2000VI) respectively. During the three weeks, NIPAAm films deposited on silicon wafers were placed in covered Petri dishes containing either deionized water or the buffer solution at room temperature $\left(\sim 22^{\circ} \mathrm{C}\right)$. To monitor the progression of changes in the chemical composition and thickness, measurements were made immediately after deposition, after rinsing in DI water to remove uncrosslinked chains, at frequent intervals during the first week of exposure, and weekly thereafter; in some cases, when the NIPAAm film had completely delaminated or degraded such that measurements were not possible, the study was terminated. NIPAAm thin films were considered to be "stable" when they showed 
no significant change in their chemical bonding structure nor a drastic reduction in thickness either due to dissolution of the polymer chains or delamination.

\section{B.1 Variable Angle Spectroscopic Ellipsometry Studies}

Thickness values of NIPAAm films prepared under different reactor conditions and exposed to DI water, PBS, and TAE solutions are shown Figs. A.1, A.2, and A.3 respectively as a function of exposure time. Data points in the figures represent the average of at least two different measurements of thickness on each sample obtained by fitting the data from variable angle spectroscopic ellipsometry to a Cauchy model layer; error bars, mostly contained within the data symbol, are the averages of the mean square error involved in fitting the data to the model. In cases where the thickness could not be determined due to film roughness/heterogeneity as a result of exposure to a liquid, the data point has been omitted from the graphs. Tables A.1, A.2, and A.3 summarize the results from the ellipsometry measurements.

From the figures and tables, it is clear that NIPAAm films behave differently in DI water and the buffer solutions. In DI water, NIPAAm films deposited at the lower substrate temperature $\left(120^{\circ} \mathrm{C}\right)$ dissolve, and therefore diminish in thickness with time; on the other hand, NIPAAm films deposited at $175^{\circ} \mathrm{C}$ showed a slight reduction in thickness of the film due to film dissolution when they were exposed to DI water immediately after deposition, but swelled in water, and remained stable for at least 3 weeks. In addition, when films deposited at the same temperature, but at different pressures are compared, 
NIPAAm films formed at 93.3 Pa swell more than the films deposited at $133.3 \mathrm{~Pa}$, and hence may be considered to be more stable.

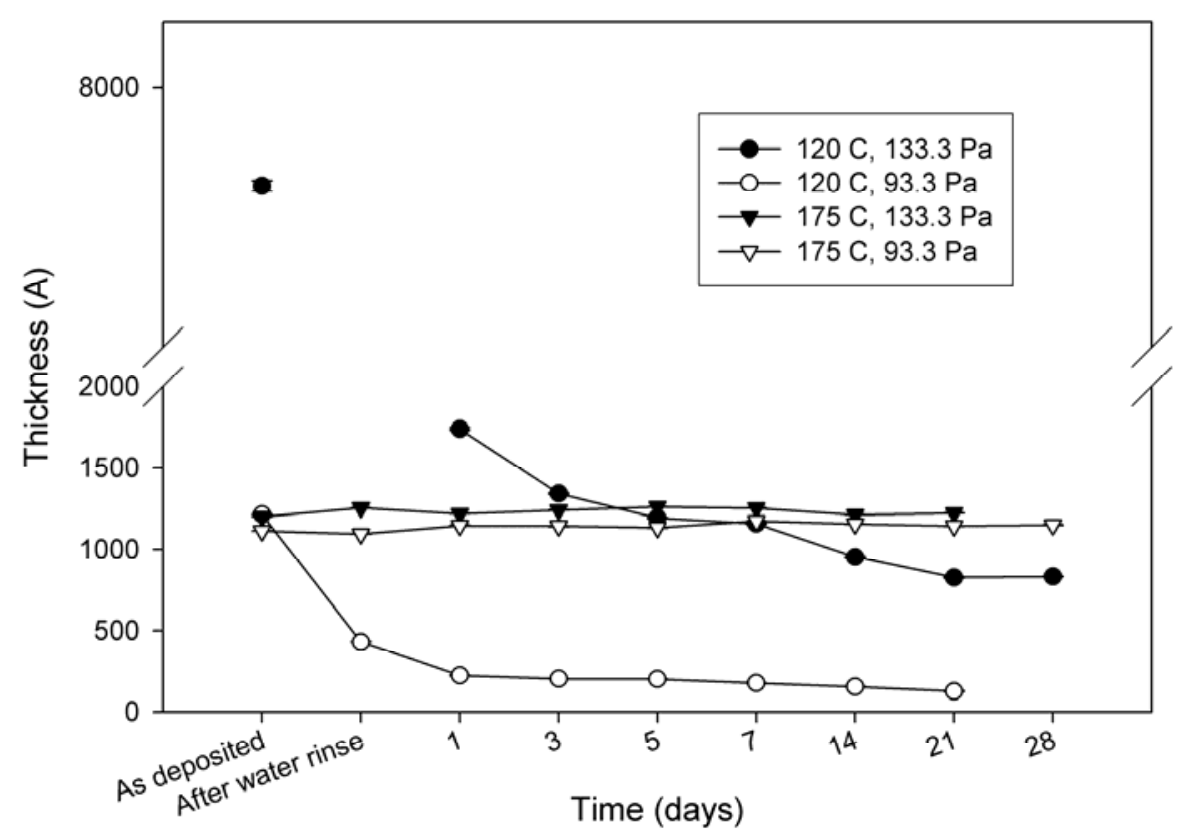

Figure B.1 Thickness of NIPAAm films prepared under different reactor conditions as a function of exposure to DI water. 


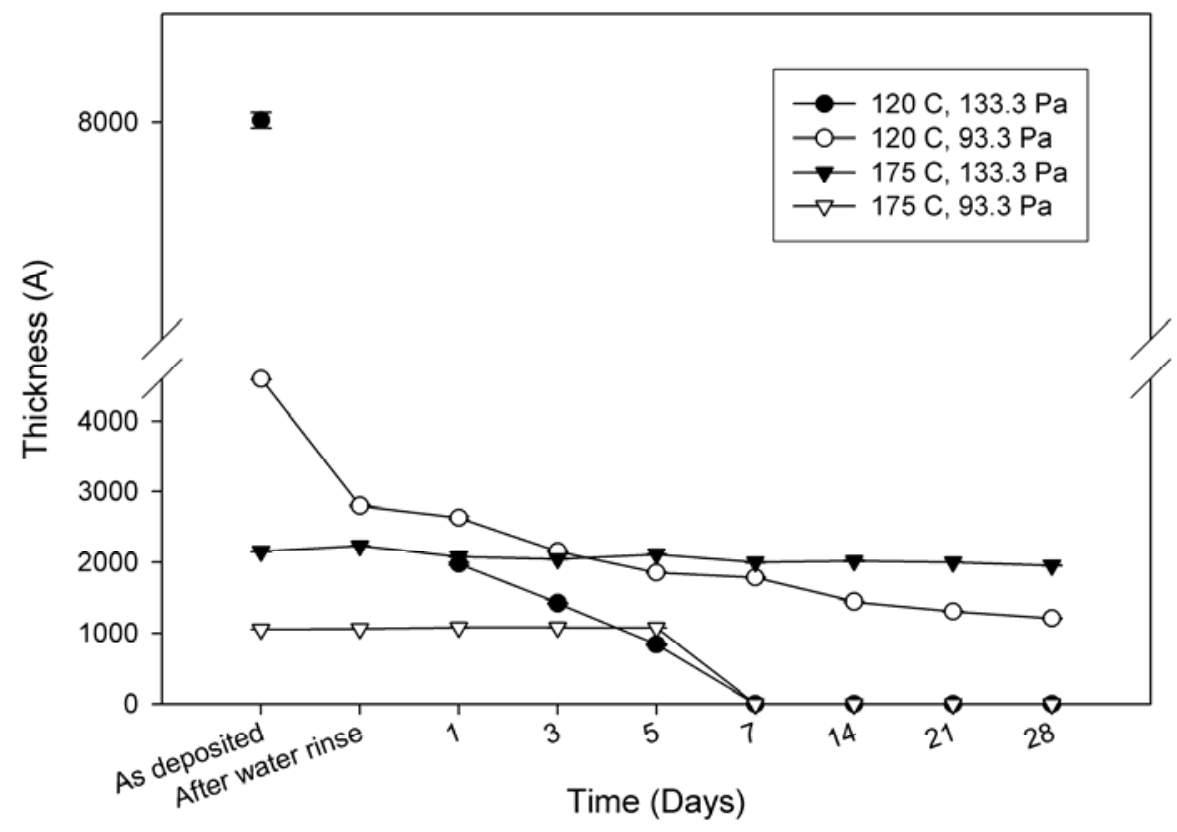

Figure B.2 Thickness of NIPAAm films prepared under different reactor conditions as a function of exposure to PBS solution.

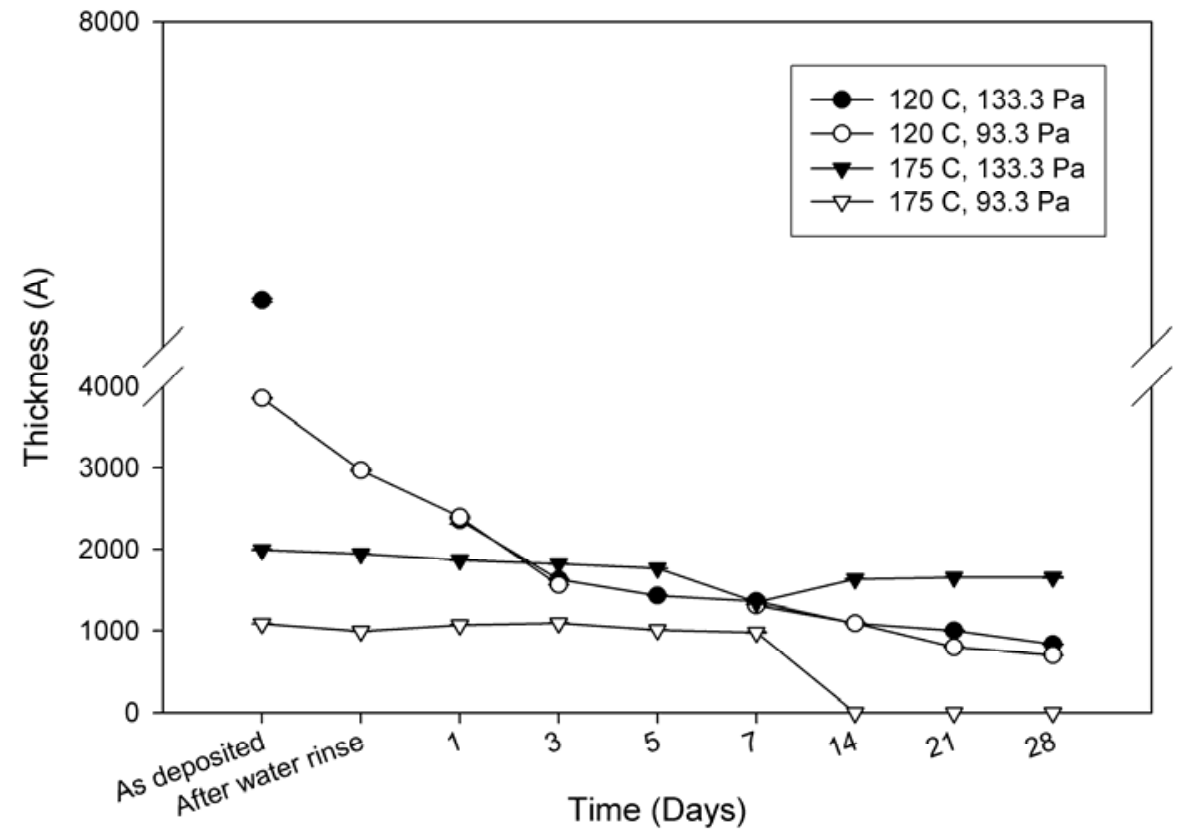

Figure B.3 Thickness of NIPAAm films prepared under different reactor conditions as a function of exposure to TAE solution. 
Table B.1 Thickness values of NIPAAm films exposed to DI water

\begin{tabular}{|c|c|c|c|c|c|c|c|c|}
\hline Studies in DI water & \multicolumn{2}{|c|}{$120^{\circ} \mathrm{C}, 133.3 \mathrm{~Pa}$} & \multicolumn{2}{|c|}{$120^{\circ} \mathrm{C}, 93.3 \mathrm{~Pa}$} & \multicolumn{2}{|c|}{$175^{\circ} \mathrm{C}, 133.3 \mathrm{~Pa}$} & \multicolumn{2}{|c|}{$175^{\circ} \mathrm{C}, 93.3 \mathrm{~Pa}$} \\
\hline Exposure time (Days) & Thickness $(\AA)$ & Error $(\AA)$ & Thickness $(\AA)$ & Error $(\AA)$ & Thickness $(\AA)$ & Error $(\AA)$ & Thickness $(\AA)$ & Error $(\AA)$ \\
\hline As deposited & 7848.16 & 8.11 & 1214.375 & 0.3 & 1200.69 & 0.324 & 1112.83 & 1.02 \\
\hline After water rinse & -- & -- & 431.12 & 0.604 & 1253.58 & 0.325 & 1095.01 & 1.23 \\
\hline 1 & 1738.71 & 8.13 & 221.05 & 0.9665 & 1219.39 & 0.275 & 1145.67 & 2.21 \\
\hline 3 & 1340.69 & 4.06 & 200.87 & 0.775 & 1240.94 & 0.34 & 1141.89 & 1.5 \\
\hline 5 & 1191.61 & 3.41 & 198.9 & 1.09 & 1260.1 & 0.37 & 1132.59 & 1.29 \\
\hline 7 & 1156.82 & 3.04 & 174.68 & 1 & 1253.24 & 0.317 & 1172.29 & 1.7 \\
\hline 14 & 952.128 & 2.24 & 153.07 & 1.04 & 1213.02 & 0.372 & 1154.17 & 1.41 \\
\hline 21 & 824.55 & 2.32 & 126.05 & 1.29 & 1222.19 & 0.955 & 1142.75 & 1.403 \\
\hline 28 & 829.56 & 1.98 & & & & & 1149.34 & 1.78 \\
\hline
\end{tabular}


Table B.2 Thickness values of NIPAAm films exposed to PBS

\begin{tabular}{|c|c|c|c|c|c|c|c|c|}
\hline Studies in PBS & \multicolumn{2}{|c|}{$120^{\circ} \mathrm{C}, 133.3 \mathrm{~Pa}$} & \multicolumn{2}{|c|}{$120^{\circ} \mathrm{C}, 93.3 \mathrm{~Pa}$} & \multicolumn{2}{|c|}{$175^{\circ} \mathrm{C}, 133.3 \mathrm{~Pa}$} & \multicolumn{2}{|c|}{$175^{\circ} \mathrm{C}, 93.3 \mathrm{~Pa}$} \\
\hline Exposure time (Days) & Thickness $(\AA)$ & Error $(\AA)$ & Thickness $(\AA)$ & Error $(\AA)$ & Thickness $(\AA)$ & Error $(\AA)$ & Thickness $(\AA)$ & Error $(\AA)$ \\
\hline As deposited & 8001.79 & 7.25 & 4597.24 & 4.10 & 2154.15 & 2.32 & 1051.71 & 0.605 \\
\hline After water rinse & -- & -- & 2800.13 & 8.85 & 2232.44 & 0.95 & 1061.11 & 0.82 \\
\hline 1 & 1978.11 & 15.7 & 2634.13 & 25.1 & 2078.04 & 1.29 & 1079.872 & 1.01 \\
\hline 3 & 1423.29 & 5.91 & 2156.99 & 6.67 & 2046.45 & 1.87 & 1080.06 & 2.13 \\
\hline 5 & 836.53 & 10 & 1851.77 & 4.49 & 2112.94 & 0.87 & 1076.36 & 0.98 \\
\hline 7 & 0 & 0 & 1782.75 & 3.42 & 1999.87 & 7.37 & 0 & 0 \\
\hline 14 & 0 & 0 & 1441.67 & 2.04 & 2020.07 & 9.58 & 0 & 0 \\
\hline 21 & 0 & 0 & 1308.05 & 2.38 & 2001.29 & 1.76 & 0 & 0 \\
\hline 28 & 0 & 0 & 1211.00 & 2.13 & 1953.99 & 2.75 & 0 & 0 \\
\hline
\end{tabular}


Table B.3 Thickness values of NIPAAm films exposed to TAE

\begin{tabular}{|c|c|c|c|c|c|c|c|c|}
\hline Studies in TAE & \multicolumn{2}{|c|}{$120^{\circ} \mathrm{C}, 133.3 \mathrm{~Pa}$} & \multicolumn{2}{|c|}{$120^{\circ} \mathrm{C}, 93.3 \mathrm{~Pa}$} & \multicolumn{2}{|c|}{$175^{\circ} \mathrm{C}, 133.3 \mathrm{~Pa}$} & \multicolumn{2}{|c|}{$175^{\circ} \mathrm{C}, 93.3 \mathrm{~Pa}$} \\
\hline Exposure time (Days) & Thickness $(\AA)$ & Error $(\AA)$ & Thickness $(\AA)$ & Error $(\AA)$ & Thickness $(\AA)$ & Error $(\AA)$ & Thickness $(\AA)$ & Error $(\AA)$ \\
\hline As deposited & 6886.799 & 6.7 & 3854.82 & 3.93 & 1990.79 & 4.09 & 1085.43 & 1.72 \\
\hline After water rinse & -- & --- & 2971.46 & 12.2 & 1941.76 & 1.24 & 992.67 & 1.04 \\
\hline 1 & 2359.17 & 45.6 & 2394.45 & 15.2 & 1864.61 & 1.53 & 1071.29 & 1.58 \\
\hline 3 & 1625.11 & 4.15 & 1559.78 & 3.09 & 1819.25 & 1.63 & 1095.60 & 1.99 \\
\hline 5 & 1424.05 & 4.99 & -- & -- & 1763.77 & 1.052 & 1008.05 & 1.44 \\
\hline 7 & 1356.48 & 5.96 & 1312.05 & 2.86 & 1344.18 & 24.3 & 977.14 & 5.64 \\
\hline 14 & 1089.16 & 4.94 & 1093.22 & 6.42 & 1633.62 & 1.12 & 0 & 0 \\
\hline 21 & 999.17 & 4.51 & 795.31 & 2.50 & 1649.39 & 0.66 & 0 & 0 \\
\hline 28 & 829.18 & 4.6 & 698.02 & 1.63 & 1649.92 & 5.41 & 0 & 0 \\
\hline
\end{tabular}


The ellipsometry results from exposure to DI water suggest that films formed at lower temperatures and higher pressures diminish in thickness upon prolonged exposure to DI water due to the lack of adequate crosslink density, whereas films formed at higher temperatures and lower pressures, believed to yield films with greater crosslink density, are able to swell in deionized water and remain stable for at least three weeks. These results support the trends presented in Chapters $3 \& 4$, and therefore confirm the previous results.

Upon exposure to biologically relevant, basic buffer solutions such as PBS and TAE all NIPAAm films studied showed a decrease in film thickness. Ironically, NIPAAm films deposited at $175^{\circ} \mathrm{C}$ and $93.3 \mathrm{~Pa}$, which were considered to be most stable based on their behavior in DI water due to their high crosslink density, completely delaminated ("lift-off") from the silicon surface within the first week after maintaining a stable thickness for a few days. NIPAAm films deposited at $120^{\circ} \mathrm{C}$ and $133.3 \mathrm{~Pa}$ showed different behavior in PBS and TAE solutions; in PBS solution, the films delaminated before the end of the first week, whereas in TAE solution, the films adhered to the underlying silicon for at least three weeks, but continued to diminish in thickness as in DI water. Furthermore, NIPAAm films deposited at $120^{\circ} \mathrm{C}, 93.3 \mathrm{~Pa}$ and $175^{\circ} \mathrm{C}, 133.3 \mathrm{~Pa}$ showed similar behavior in both buffer solutions and demonstrated partial delamination at the edges and corners after approximately five days, and a reduction in thickness of the remaining film with exposure time. In summary, exposure to the basic buffers resulted in delamination of highly crosslinked NIPAAm films; the less crosslinked films dissolved in the buffers, but did not delaminate. 
Initially, delamination of highly crosslinked NIPAAm films relative to less crosslinked films was believed to occur due to differences in chemical bonding at the silicon and NIPAAm interface. However, when the interface between the NIPAAm film and silicon substrate was probed using core level and valence band x-ray photoelectron spectra obtained at high take off angles relative to the substrate $\left(\sim 75^{\circ}\right)$, the differences in the interfacial bonding in NIPAAm films prepared under different reactor conditions were not obvious. It is conceivable that delamination occurs in highly crosslinked films because of the scarcity of uncrosslinked chains in the network; in other words, cohesion in the network may be greater than adhesion at the interface of silicon and NIPAAm. In contrast, in less crosslinked NIPAAm films, due to the abundance of uncrosslinked chains in the hydrogel network, prolonged exposure to buffer solutions may result in dissolution, but not delamination. Furthermore, residual stress built into the NIPAAm films during plasma polymerization at elevated temperatures, thermal expansion mismatch between the NIPAAm film and the substrate, stress imposed on the hydrogel network because of water induced swelling, and salt deposition in the network from exposure to buffer solutions (absent in the case of DI water) may also lead to delamination of highly crosslinked films at the silicon-NIPAAm interface. Indeed, delamination from the edges, as observed in highly-crosslinked NIPAAm films, has been reported previously, and is believed to occur because of the interfacial stress between the thin film and substrate resulting from various intrinsic stresses thin film, and the freeedge effect ${ }^{220-223}$. The free-edge effect arises when residual/extrinsic stresses in the thin 
film exert several out-of-plane stresses that are normal to the interface at the edges, which can initiate delamination.

In all the preceding experiments, the entire sample, consisting of a NIPAAm film on a silicon substrate, was immersed in the DI water and buffer solutions, thereby allowing exposure to the interface between the NIPAAm film and the silicon substrate at the edges. In order to determine if the exposure to the interface at the edges was necessary for delamination, a separate set of experiments were conducted. Two drops $(10 \mu \mathrm{l})$ of PBS and TAE solutions were placed in the center of two separate samples of NIPAAm films formed at $175^{\circ} \mathrm{C}$ and $93.3 \mathrm{~Pa}$, and were stored in separate, closed Petri dishes at room temperature $\left(\sim 22^{\circ} \mathrm{C}\right)$ for a period of two weeks. The condition of the films was monitored under the same schedule described previously. While the thickness in the area of the NIPAAm films in contact with the buffer solutions decreased, no delamination was observed, even after two weeks of continuous exposure. This suggests that exposure of the interface between NIPAAm and silicon at the edges, and alignment of the thin film edge with the substrate's edge may be necessary for delamination of highly crosslinked NIPAAm films, such as the films prepared at $175^{\circ} \mathrm{C}$ and $93.3 \mathrm{~Pa}$. Although the basic buffer solutions may have access to the interface between NIPAAm and silicon when they penetrate the film through diffusion, any attack at the interface may not be sufficient to delaminate the highly crosslinked network due to the lack of exposed edges. Hence, the free-edge effect, which causes delamination to initiate preferentially at the edges, may be important in the delamination of highly crosslinked NIPAAm films from the substrate ${ }^{221}$. 


\section{B.2 Fourier Transform Infrared Spectroscopy studies}

To study the changes in chemical bonding characteristics of the NIPAAm films exposed to different aqueous environments, Fourier transform infrared (FTIR) spectra of the films were obtained as a function of exposure to DI water (Figure A.4), PBS (Figure A.5), and TAE (Figure A.6). The most significant changes in the FTIR spectra occurred in the amide I (-C=O stretch) and II (-N-H deformation) bands as a result of changes in the degree of hydrogen bonding. Based on the analysis of FTIR spectra presented in Chapter 2, it is clear that NIPAAm thin films formed at lower substrate temperatures and higher operating pressures show greater intramolecular hydrogen bonding than the films formed at higher substrate temperatures and lower pressures. In the presence of strong hydrogen bonding, compared to the cases when strong hydrogen bonding is absent, the amide I band is found at lower wavenumbers, whereas the amide II band is found at higher wavenumbers. Table A.4 gives a comparison of the positions of amide I and II bands in FTIR spectra of various NIPAAm films, exposed to DI water, PBS and TAE, and obtained immediately after deposition and at the end of the study. 


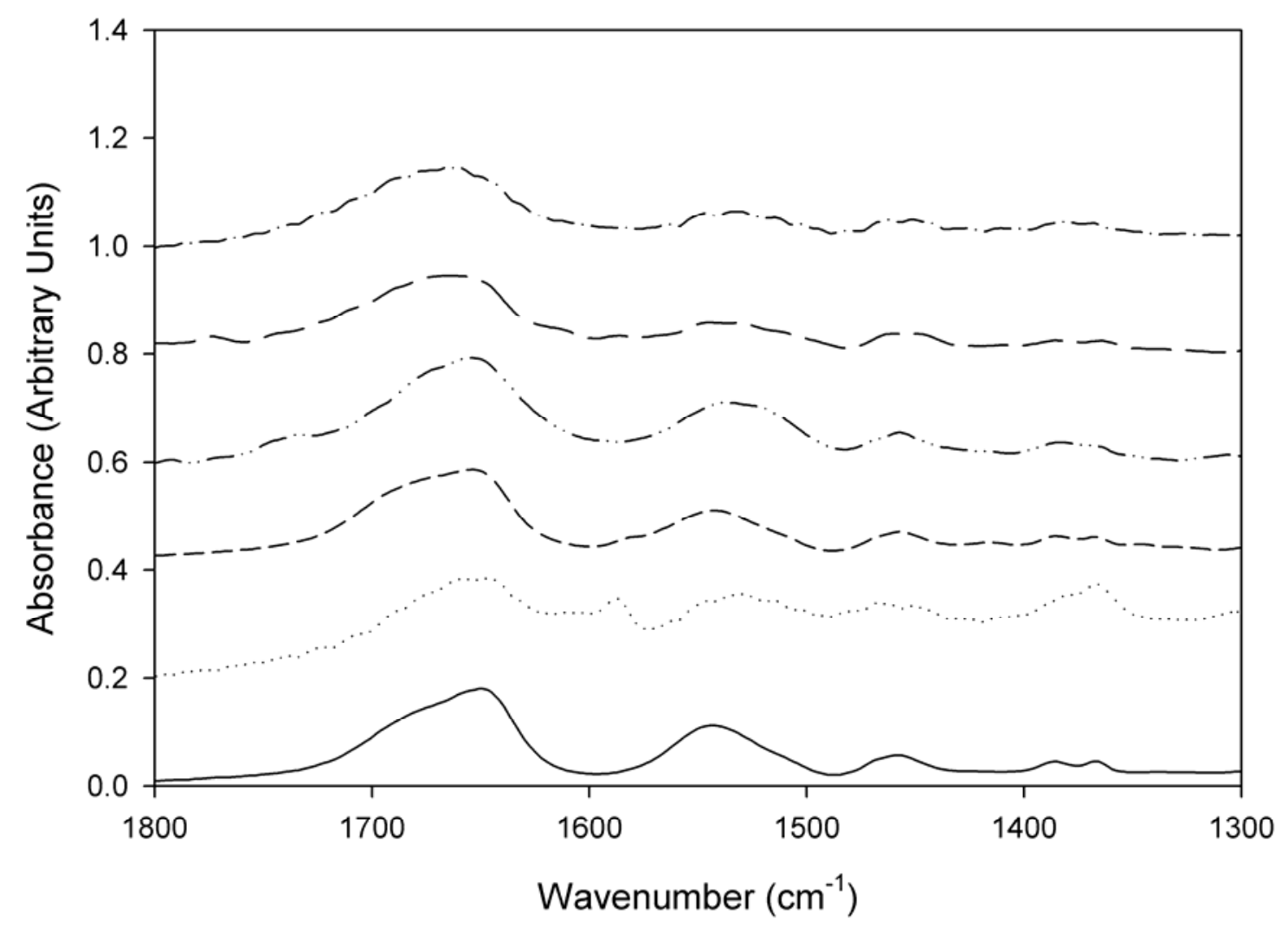

\begin{tabular}{|c|c|}
\hline & $120 \mathrm{C}, 133.3 \mathrm{~Pa}-$ After deposition \\
\hline & $120 \mathrm{C}, 133.3 \mathrm{~Pa}-$ After 21 days \\
\hline-- & $120 \mathrm{C}, 93.3 \mathrm{~Pa}$ - After deposition \\
\hline$-\cdots-\cdots$ & $120 \mathrm{C}, 93.3 \mathrm{~Pa}-$ After 21 days \\
\hline-- & $175 \mathrm{C}, 93.3 \mathrm{~Pa}$ - After deposition \\
\hline$-1-1$ & $175 \mathrm{C}, 93.3 \mathrm{~Pa}-$ After 21 days \\
\hline
\end{tabular}

Figure B.4. FTIR spectra of NIPAAm films exposed to DI water showing the amide I and II regions. 


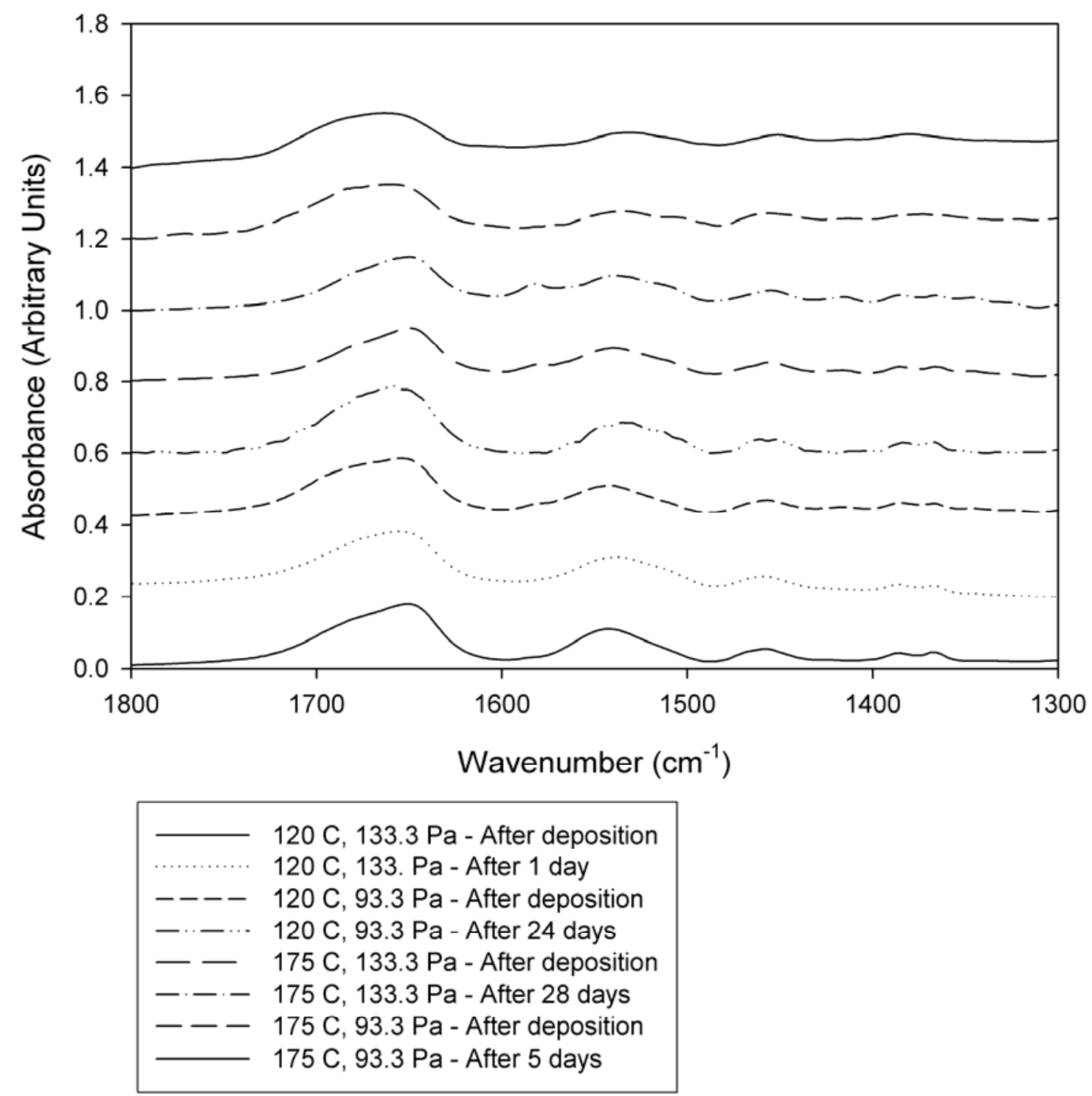

Figure B.5. FTIR spectra of NIPAAm films exposed to PBS solution showing the amide I and II regions. 


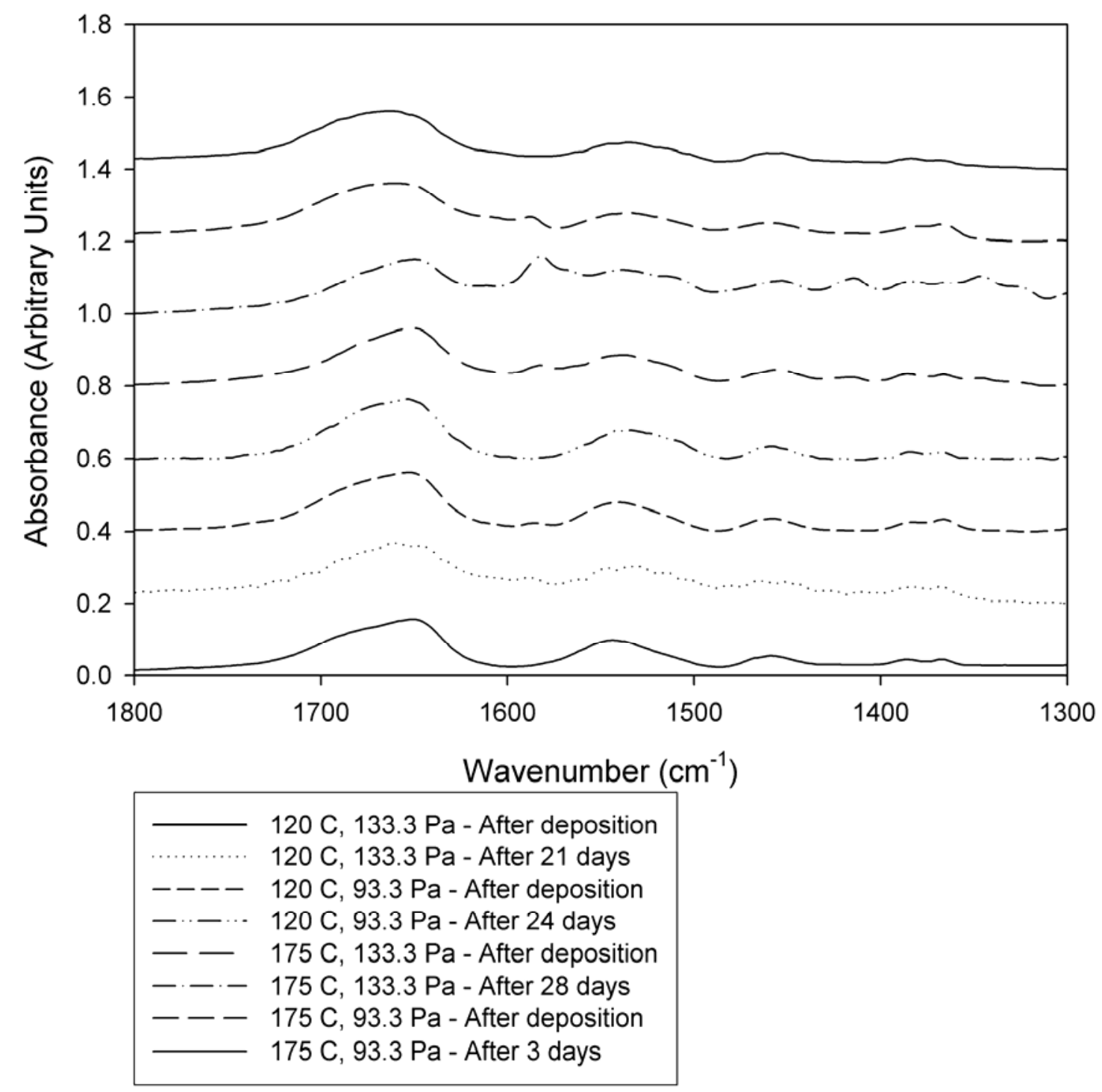

Figure B.6. FTIR spectra of NIPAAm films exposed to TAE solution showing the amide I and II regions. 
Table B.4 Comparison of Amide I and II bands in the FTIR spectra

\begin{tabular}{|c|c|c|c|c|}
\hline \multirow{7}{*}{ Studies in Water } & & & Amide I $\left(\mathrm{cm}^{-1}\right)$ & Amide II $\left(\mathrm{cm}^{-1}\right)$ \\
\hline & \multirow{2}{*}{$120 \mathrm{C}, 133.3 \mathrm{~Pa}$} & After deposition & 1649 & 1543 \\
\hline & & After 21 days & 1649 & 1529 \\
\hline & \multirow{2}{*}{$120 \mathrm{C}, 93.3 \mathrm{~Pa}$} & After deposition & 1655 & 1543 \\
\hline & & After 21 days & 1655 & 1537 \\
\hline & \multirow{2}{*}{$175 \mathrm{C}, 93.3 \mathrm{~Pa}$} & After deposition & 1664 & 1547 \\
\hline & & After 21 days & 1664 & 1531 \\
\hline \multirow{8}{*}{ Studies in PBS } & \multirow{2}{*}{$120 \mathrm{C}, 133.3 \mathrm{~Pa}$} & After deposition & 1651 & 1543 \\
\hline & & After 1 day & 1657 & 1537 \\
\hline & \multirow{2}{*}{$120 \mathrm{C}, 93.3 \mathrm{~Pa}$} & After deposition & 1655 & 1543 \\
\hline & & After 24 days & 1659 & 1535 \\
\hline & \multirow{2}{*}{$175 \mathrm{C}, 133.3 \mathrm{~Pa}$} & After deposition & 1651 & 1539 \\
\hline & & After 28 days & 1651 & 1537 \\
\hline & \multirow{2}{*}{$175 \mathrm{C}, 93.3 \mathrm{~Pa}$} & After deposition & 1660 & 1533 \\
\hline & & After 5 days & 1663 & 1533 \\
\hline \multirow{8}{*}{ Studies in TAE } & \multirow{2}{*}{$120 \mathrm{C}, 133.3 \mathrm{~Pa}$} & After deposition & 1651 & 1545 \\
\hline & & After 21 days & 1659 & 1531 \\
\hline & \multirow{2}{*}{$120 \mathrm{C}, 93.3 \mathrm{~Pa}$} & After deposition & 1653 & 1541 \\
\hline & & After 24 days & 1655 & 1533 \\
\hline & \multirow{2}{*}{$175 \mathrm{C}, 133.3 \mathrm{~Pa}$} & After deposition & 1651 & 1538 \\
\hline & & After 28 days & 1649 & 1537 \\
\hline & \multirow{2}{*}{$175 \mathrm{C}, 93.3 \mathrm{~Pa}$} & After deposition & 1662 & 1535 \\
\hline & & After 3 days & 1664 & 1535 \\
\hline
\end{tabular}

After prolonged exposure to water, in all the NIPAAm films studied, the amide II band shifts to lower wavenumbers, suggesting partial loss of intramolecular hydrogen bonding. On the other hand, after exposure to the buffer solutions PBS and TAE, amide I 
and II bands shifted to higher and lower wavenumbers respectively, suggesting that the intramolecular hydrogen bonding was diminished. Of course, the shifts in wavenumberswere greatest in NIPAAm films which showed the greatest degree of hydrogen bonding immediately after deposition. Diminished hydrogen bonding as a result of exposure to basic buffer solutions was expected because bases are known to disrupt hydrogen bonding. The subsequent loss of physical crosslinks in the hydrogel network provided by the hydrogen bonds causes greater dissolution of NIPAAm films in basic solutions relative to DI water; the ellipsometry results presented in the previous section support this trend. However, it has not been possible to explain the loss of hydrogen bonding, evidenced by the shift of amide II band to lower wavenumbers, upon exposure to DI water.

In summary, if long term stability and greater swelling capacity in DI water is required, NIPAAm films of the greatest crosslink density (of the films investigated in this study), formed at $175^{\circ} \mathrm{C}$ and $93.3 \mathrm{~Pa}$ are suitable. In basic buffer solutions, NIPAAm films of high crosslink density may be considered to be stable, although they show small decreased in thickness with prolonged exposure. However, if the interface between highly crosslinked NIPAAm and silicon is exposed at the edge, the films delaminate completely within a week of exposure to basic buffers. In contrast, with prolonged exposure to both DI water and basic buffer solutions, NIPAAm films of low crosslink density, such as films prepared at a substrate temperature of $120^{\circ} \mathrm{C}$ and reactor pressure of $133.3 \mathrm{~Pa}$, show large reduction in thickness values, possibly due to dissolution, but do not delaminate even when the NIPAAm film-substrate interface is exposed at the edge. 
Due to the existence of adequate crosslinks, unlike NIPAAm films of lower crosslink density, NIPAAm films of higher crosslink density do not demonstrate high levels of dissolution in aqueous solutions. However, the free-edge effect, and interfacial attack by salt containing buffer solutions cause delamination in highly crosslinked NIPAAm films. Therefore, to ensure good stability, NIPAAm films of adequate crosslink density are required to prevent dissolution; however, prolonged exposure of the edges of the interface between NIPAAm and silicon must be avoided to prevent delamination. 


\section{REFERENCES}

1. Hoffman, A. S.; Stayton, P. S.; Bulmus, V.; Chen, G.; Chen, J.; Cheung, C.; Chilkoti, A.; Ding, Z.; Dong, L.; Fong, R.; Lackey, C. A.; Long, C. J.; Miura, M.; Morris, J. E.; Murthy, N.; Nabeshima, Y.; Park, T. G.; Press, O. W.; Shimoboji, T.; Shoemaker, S.; Yang, H., Joon; Monji, N.; Nowinski, R., C.; Cole, C. A.; Priest, J., H.; Harris, M., J.; Nakamae, K.; Nishino, T.; Miyata, T., Really smart bioconjugatesof smart polymers and receptor proteins. J. Biomed. Mater. Res. 2000, 52, 577-586.

2. $\quad$ van Veen, A.; Schut, H.; de Vries, J.; Hakvoort, R. A.; IJpma, M. R. In Analysis of positron profiling data by means of 'VEPFIT', 4th Interntional Workshop on slowpositron beam techniques for solids and surfaces, London, Ont. Canada, 1991; London, Ont. Canada, 1991; pp 171-196.

3. Kay, E.; Coburn, J.; Dilks, A., Plasma chemistry of fluorocarbons as related to plasma etching and plasma polymerization. In Plasma chemistry III, Veprek, S.;

Venugopalan, M., Eds. Springer Berlin/ Heidelberg: 1980; Vol. 94, pp 1-42.

4. http://positrons.physics.lsa.umich.edu/solids/nanopos/PALSintro/PALS\%20basics.htm, In Accessed in Jul. 2006.

5. Zhang, R.; Cao, H.; Chen, H. M.; Mallon, P.; Sandreczki, T. C.; Richardson, J. R.; Jean, Y. C.; Nielsen, B.; Suzuki, R.; Ohdaira, T., Development of positron annihilation spectroscopy to test accelerated weathering of protective polymer coatings. Rad. Phys. Chem. 2000, 58, 639-644.

6. Eichenbaum, G. M.; Kiser, P. F.; Simon, S. A.; Needham, D., pH and IonTriggered Volume Response of Anionic Hydrogel Microspheres. Macromol. 1998, 31, 5084-5093.

7. Schild, H. G., Poly(N-Isopropylacrylamide): Experiment, theory and application. Prog. Polym. Sci. 1992, 17, 163-249.

8. Shiga, T., Deformation and viscoelastic behavior of polymer gels in electric fields. Adv. Polym. Sci. 1997, 134, 133-162. 
9. Hoffman, A., S., Intelligent polymers ( in medicine and biotechnology). In Encyclopedia of polymeric materials, CRC Press: 1996; pp 3282-3292.

10. Galaev, I., Y. ; Mattiason, B., 'Smart' polymers and what they could do in biotechnology and medicine. TIBTECH 1999, 17, 335-340.

11. Galaev, I. Y.; Gupta, M. N.; Mattiasson, B., Use smart polymers for bioseparations. CHEMTECH 1996, (December), 19-25.

12. Nath, N.; Chilkoti, A., Creating "smart" surfaces using stimuli responsive polymers. Advanced materials 2002, 14, (17), 1243-1247.

13. Cheng, X.; Wang, Y.; Hanein, Y.; Boehringer, K. F.; Ratner, B. D., Novel cell patterning using microheater-controlled thermoresponsive plasma films. J. Biomed. Mater. Res. A 2004, 70A, (2), 159-168.

14. Yamada, N.; Okano, T.; Sakai, H.; Karikusa, F.; Sawasaki, Y.; Sakurai, Y., Thermo-responsive polymeric surfaces; control of attachment and detachment of cultured cells. Makromol. Chem., Rapid Commun. 1990, 11, 571-576.

15. Balamurugan, S.; Mendez, S.; Balamurugan, S. S.; O' Brien II, M. J.; Lopez, G., Thermal response of Poly(N-Isopropylacrylamide) brushes probed by surface plasmon resonance. Langmuir 2003, 19, 2545-2549.

16. Liang, L.; Feng, X.; Liu, J.; Rieke, P. C., Preparation of composite-crosslinked Poly(N-Isopropylacrylamide) gel layer and characteristics of reverse hydrophilichydrophobic surface. J. Appl. Polym. Sci. 1999, 72, 1-11.

17. Kuckling, D. A., Hans-Jurgen, P.; Arndt, Karl-Friedrich; Hoffmann, Jan; Plotner, Matthias and Wolff, Thomas, Photocrosslinking of thin films of temperature-sensitive polymers. Polymers for advanced technologies 1999, 10, 345-352.

18. Harmon, M. E.; Jakob, T. A. M.; Knoll, W.; Frank, C. W., A surface plasmon resonance study of volume phase transitions in $\mathrm{N}$-isopropylacrylamide gel films. Macromol. 2002, 35, 5999-6004.

19. Pan, V. Y.; Wesley, R. A.; Luginbuhl, R.; Denton, D. D.; Ratner, B. D., Plasma polymerized N-Isopropylacrylamide: Synthesis and characterization of a smart thermally responsive coating. Biomacromol. 2001, 2, 32-36. 
20. Teare, D. O. H.; Barwick, D. C.; Schofield, W. C. E.; Garrod, R. P.; Beeby, A.; Badyal, J. P. S., Functionalization of solid surfaces with thermoresponsive proteinresistant films. J. Phys. Chem. B 2005, 109, 22407-22412.

21. Tamirisa, P. A.; Koskinen, J.; Hess, D. W., Plasma polymerized hydrogel thin films. Thin Solid Films 2006, (in press).

22. Lieberman, M. A.; Lichtenberg, A. J., Principles of plasma discharges and materials processing. 2nd ed.; John Wiley \& Sons Inc.: Hoboken, New Jersey, 2005.

23. Morosoff, N., An introduction to Plasma polymerization. In Plasma deposition, treatment, and etching of polymers, d'Agostino, R., Ed. Academic Press, Inc.: San Diego, CA, 1990; pp 1-93.

24. Yasuda, H., Plasma polymerization. Academic Press Inc.: Orlando, FL, 1985.

25. Yasuda, H.; Yasuda, T., The competitive ablation and polymerization (CAP) principle and the plasma sensitivity of elements in plasma polymerization and treatment. J. Polym. Sci. Part A: Polym. Chem. 2000, 38, 943-953.

26. Lopez, G. P.; Ratner, B. D., Substrate temperature effects on film chemistry in plasma depositions of organics. II. Polymerizable precursors. J. Polym. Chem. A 1992, $30,2415-2425$.

27. Agraharam, S.; Hess, D. W.; Kohl, P. A.; Bidstrup Allen, S. A., Plasma chemistry in fluorocarbon film deposition from pentafluoroethane/argon mixtures. J. Vac. Sci. Tech. A: Vacuum, Surfaces and Films 1999, 17, (6), 3265-3271.

28. Doblhoffer, K.; Durr, W.; Jauch, M., Electrochemical redox response of Iron (II)/(III) ions implanted in permeable, polymeric electrode coatings. Electrochim. Acta 1982, 27, (6), 677-682.

29. Bieg, K. W., Hydrocarbon plasma emission as a means to monitor plasma polymerization. Thin Solid Films 1981, 84, 411-417.

30. Doucoure, A.; Guizard, C.; Durand, J.; Berjoan, R.; Cot, L., Plasma polymerization of fluorinated monomers on mesoporous silica membranes and application to gas permeation. Journal of Membrane Science 1996, 117, (1-2), 143-150. 
31. Lee, S. H.; Lee, D. C., Preparation and characterization of thin films by plasma polymerization of hexamethyldisiloxane. Thin Solid Films 1998, 325, (1,2), 83-86.

32. Prikryl, R.; Cech, V.; Zajickova, L.; Vanek, J.; Behzadi, S.; Jones, F. R., Mechanical and optical properties of plasma-polymerized vinyltriethoxysilane. Surface and Coatings Technology 2005, 200, (1-4), 468-471.

33. Jeon, H. S.; Wyatt, J.; Harper-Nixon, D.; Weinkauf, D. H., Characterization of thin polymer-like films formed by plasma polymerization of methylmethacrylate: A neutron reflectivity study. Journal of Polymer Science, Part B: Polymer Physics 2004, 42, (13), 2522-2530.

34. Havens, M. R.; Mayhan, K. G.; James, W. J.; Schmidt, P., Plasma-deposited polymer films. I. Low-angle X-ray study. J. Appl. Polym. Sci. 1978, 22, 2793-2798.

35. Shi, F. F., Recent advances in polymer thin films prepared by plasma polymerization: Synthesis, structural characterization, properties, and applications. Surf. Coat Tech. 1996, 82, 1-15.

36. Yasuda, H.; Hsu, T., Some aspects of plasma polymerization investigated by pulsed R.F. discharge. J. Polym. Sci.: Polym. Chem. Ed. 1977, 15, 81-97.

37. Leich, M. A.; Mackie, N. M.; Williams, K. L.; Fisher, E. R., Pulsed plasma polymerization of benzaldehyde for retention of the aldehyde functional group. Macromol. 1998, 31, 7618-7626.

38. Mackie, N. M.; Castner, D. G.; Fisher, E. R., Characterization of pulsed-plasmapolymerized aromatic films. Langmuir 1998, 14, 1227-1235.

39. Groenewoud, L. M. H.; Engbers, G. H. M.; Terlingen, J. G. A.; Wormeester, H.; Feijen, J., Pulsed plasma polymerization of thiophene. Langmuir 2000, 16, 6278-6286.

40. Han, L. M.; Timmons, R. B.; Lee, W. W., Pulsed plasma polymerization of an aromatic perfluorocarbon monomer: Formation of low dielectric constant, high thermal stability films. J. Vac. Sci. Technol. B 2000, 18, (2), 799-804.

41. Labelle, C. B.; Gleason, K. K., Pulsed plasma-enhanced chemical vapor deposition from $\mathrm{CH}{ }_{2} \mathrm{~F} \_2, \mathrm{C} \_2 \mathrm{H} \_2 \mathrm{~F} \_4$, and CHClF_2.J. Vac. Sci. Technol. A 1999, 17, (2), 445-452. 
42. Biederman, H., Plasma polymer films. Imperial College Press: London, 2004.

43. Huglin, M. B.; Zakaria, M. B., Swelling properties of copolymeric hydrogels prepared by gamma irradiation. J. Appl. Polym. Sci. 1986, 31, 457.

44. Chapiro, A., Radiation chemistry of polymeric systems. Interscience New York, 1962.

45. Peppas, N. A., Hydrogels in medicine and pharmacy. CRC Press: Boca Raton, FL, 1987.

46. Tanaka, T.; Fillmore, D.; Sun, S.-T.; Nishio, I.; Swislow, G.; Shah, A., Phase Transitions in Ionic Gels. Phys. Rev. Lett. 1980, 45, 1636-1639.

47. Juodkazis, S.; Mukai, N.; Wakaki, R.; Yamaguchi, A.; Matsui, S.; Misawa, H., Reversible phase transitions in polymer gels induced by radiation forces Nature 2000, 408, 178-181.

48. Suzuki, A.; Isii, T., Phase coexistence of neutral polymer gels under mechanical constraints. J. Chem. Phys. 1999, 110, (4), 2289-2296.

49. Dusek, K.; Patterson, D., Transition in swollen polymer networks induced by intramolecular condensation. J. Polym. Sci. Part A-2 1968, 6, 1209-1216.

50. Maurer, G.; Prausnitz, J. M., Thermodynamics of phase equilibrium for systems containing gels. Fluid Phase Equilibria 1996, 115, 113-133.

51. Marchetti, M. P., S. and Cussler, E. L., Thermodynamic predictions of volume changes in temperature-sensitive gels. 2. Experiments. Macromolecules 1990, 23, 34453450 .

52. Marchetti, M. P., S. and Cussler, E. L., Thermodynamic predictions of volume changes in temperature-sensitive gels. 1. Theory. Macromolecules 1990, 23, 1760-1765.

53. Otake, K. I., Hiroshi; Konno, Mikio and Saito, Shozaburo, A new model for the thermally induced volume phase transition of gels. J. Chem. Phys. 1989, 91, (2), 13451350. 
54. Wu, S.; Li, H.; Chen, J. P.; Lam, K. Y., Modeling investigation of hydrogel volume transition. Macromol. Theory Simul. 2004, 13, 13-29.

55. Zhao, J.-H.; Kiene, M.; Hu, C.; Ho, P. S., Thermal stress and glass transition of ultrathin polystyrene films. Appl. Phys. Lett. 2000, 77, (18), 2843-2845.

56. Tanaka, T.; Hocker, L. O.; Benedek, G. B., Spectrum of light scattered from a viscoelastic gel. J. Chem. Phys. 1973, 59, (9), 5151-5159.

57. Lustig, S. R.; Caruthers, J. M.; Peppas, N. A., Continuum thermodynamics and transport theory for polymer-fluid mixtures. Chem. Eng. Sci. 1992, 47, (12), 3037-3057.

58. Grimshaw, P. E.; Nussbaum, J. H.; Grodzinsky, A. J.; Yarmush, M. L., Kinetics of electrically and chemically induced swelling in polyelectrolyte gels. J. Chem. Phys. 1990, 93, (6), 4462-4472.

59. Hariharan, D.; Peppas, N. A., Modelling of water transport in ionic hydrophilic polymers. J. Polym. Sci.:Part B: Polym. Phys. 1994, 32, 1093-1103.

60. Beyer, D.; Knoll, W.; Ringsdorf, H.; Wang, J.-H.; Timmons, R. B.; Sluka, P., Reduced protein adsorption on plastics via direct plasma deposition of triethylene glycol monoallyl ether. J. Biomed. Mater. Res. 1997, 36, 181-189.

61. Lopez, G., P. ; Ratner, B., D. ; Rapoza, R., J. ; Horbett, T., A., Plasma deposition of ultrathin films of poly(2-hydroxyethylmethacrylate): surface analysis and protein adsorption measurements. Macromol. 1993, 26, (13), 3247-3253.

62. Morra , M.; Cassinelli, C., Surface field of forces and protein adsorption behavior of poly(hydroxyethylmethacrylate) films deposited from plasma. J. Biomed. Mater. Res. 1995, 29, 39-45.

63. Tarducci, C.; Schofield, W. C. E.; Badyal, J. P. S., Monomolecular functionalization of pulsed plasma deposited poly(2-hydroxyethyl methacrylate) surfaces. Chem. Mater. 2002, 14, (6), 2541-2545.

64. Denes, A. R.; Somers, E. B.; Wong, A. C. L.; Denes, F., 12-Crown-4-ether and tri(ethylene glycol) dimethyl-ether plasma-coated stainless steel surfaces and their ability to reduce bacterial biofilm deposition. J. Appl. Polym. Sci. 2001, 81, 3425-2438. 
65. Johnston, E. E.; Bryers, J. D.; Ratner, B. D., Plasma deposition and surface characterization of oligoglyme, dioxane, and crown ether nonfouling films. Langmuir 2005, 21, 870-881.

66. Pan, V. Y.; McDevitt, T. C.; Kim, T. K.; Leach-Scampavia, D.; Stayton, P. S.; Denton, D. D.; Ratner, B. D., Micro-scale cell patterning on nonfouling plasma polymerized tetraglyme coatings by protein microcontact printing. Plasmas and polymers 2002, 7, (2), 171-183.

67. Shen, M.; Wagner, M. S.; Castner, D. G.; Ratner, B. D.; Horbett, T. A., Multivariate surface analysis of plasma-deposited tetraglyme for reduction of protein adsorption and monocyte adhesion. Langmuir 2003, 19, 1692-1699.

68. Wu, Y. J.; Timmons, R. B.; Jen, J. S.; Molock, F. E., Non-fouling surfaces produced by gas phase pulsed plasma polymerization of an ultra low molecular weight ethylene oxide containing monomer. Colloids Surf. B 2000, 18, 235-248.

69. Zhang, Z.; Menges, B.; Timmons, R. B.; Knoll, W.; Forch, R., Surface plasmon resonance studies of protein binding on plasma polymerized di(ethylene glycol) monovinyl ether films. Langmuir 2003, 19, 4765-4770.

70. Canavan, H. E.; Cheng, X.; Graham, D. J.; Ratner, B. D.; Castner, D. G., Surface characterization of the extracellular matrix remaining after cell detachment from a thermoresponsive polymer. Langmuir 2005, 21, 1949-1955.

71. Cheng, X.; Canavan, H. E.; Stein, J. M.; Hull, J. R.; Kweskin, S. J.; Wagner, M. S.; Somorjai, G. A.; Castner, D. G.; Ratner, B. D., Surface chemical and mechanical properties of plasma-polymerized N-Isopropylacrylamide. Langmuir 2005, 21, (17), 7833-7841.

72. Hatakeyama, H.; Kikuchi, A.; Yamamoto, M.; Okano, T., Bio-functionalized thermoresponsive interfaces facilitating cell adhesion and proliferation. Biomat. 2006, 27, (29), 5069-5078

73. Lynch, I.; Blute, I. A.; Zhmud, B.; MacArtain, P.; Tosetto, M.; Allen, L. T.; Byrne, H. J.; Farrell, G. F.; Keenan, A. K.; Gallagher, W. M.; Dawson, K. A., Correlation of the adhesive properties of cells to N-Isopropylacrylamide/N-tert-Butylacrylamide copolymer surfaces with changes in surface structure using contact angle measuremetns, molecular simulations, and Raman spectroscopy. Chem. Mat. 2005, 17, (15), 3889-3898. 
74. Hatakeyama, H.; Kikuchi, A.; Yamamoto, M.; Okano, T., Influence of insulin immobilization to thermoresponsive culture surfaces on cell proliferation and thermally induced cell detachment. Biomat. 2005, 26, (25), 5167-5176.

75. Ohya, S.; Kidoaki, S.; Matsuda, T., Poly(N-Isopropylacrylamide) (PNIPAM)grafted gelatin hydrogel surfaces: interrelationship between microscopic structure and mechanical property of surface regions and cell adhesiveness. Biomat. 2005, 26, (16), 3105-3111.

76. Schmaljohann, D.; Oswald, J.; Jorgensen, B.; Nitschke, M.; Beyerlein, D.; Werner, C., Thermo-responsive PNiPAAm-g-PEG films for controlled cell detachment. Biomacromol. 2003, 4, 1733-1739.

77. Allen, L. T.; Fox, E. J. P.; Blute, I. A.; Kelly, Z. D.; Rochev, Y.; Keenan, A. K.; Dawson, K. A.; Gallagher, W. M., Interaction of soft condensed materials with living cells: Phenotype/transcriptome correlations for the hydrophobic effect. Proc. Natl. Acad. Sci. 2003, 100, (11), 6331-6336.

78. Grate, J. W., Acoustic wave microsensor arrays for vapor sensing. Chem. Rev. 2000, $100,2627-2648$.

79. McGill, A. R.; Abraham, M. H.; Grate, J. W., Choosing polymer coatings for chemical sensors. CHEMTECH 1994, 24, (9), 27-37.

80. Beebe, D. J.; Moore, J. S.; Bauer, J. M.; Yu, O.; Liu, R. H.; Devadoss, C.; Jo, B.H., Functional hydrogel structures for autonomous flow control inside microfluidic channels. Nature 2000, 404, 588-590.

81. van der Linden, H. J.; Herber, S.; Olthius, W.; Bergveld, P., Stimulus-sensitive hydrogels and their applications in chemical (micro)analysis. The Analyst 2003, 128, 325331.

82. Sheppard Jr., N. F.; Lesho, M. J.; McNally, P.; Francomacaro, A. S., Microfabricated conductimetric $\mathrm{pH}$ sensor. Sensors and Actuators B 1995, 28, 95-102.

83. Gerlach, G.; Guenther, M.; Sorber, J.; Suchanek, G.; Arndt, K.-F.; Richter, A., Chemical and $\mathrm{pH}$ sensors based on the swelling behavior of hydrogels. Sensors and Actuators B 2005, 111-112, 555-561. 
84. Marshall, A. J.; Blyth, J.; Davidson, C. A. B.; Lowe, C. R., pH-sensitive holographic sensors. Anal. Chem. 2003, 75, 4423-4431.

85. Richter, A.; Bund, A.; Keller, M.; Arndt, K.-F., Characterization of a microgravimetric sensor based on $\mathrm{pH}$ sensitive hydrogels. Sensors and Actuators $B$ 2004, 99, 579-585.

86. Miyata, T.; Uragami, T.; Nakame, K., Biomolecule-sensitive hydrogels. $A d v$. Drug Del. Rev. 2002, 54, 79-98.

87. Barnes, C.; D'Silva, C.; Jones, J. P.; Lewis, T. J., A concanavalin A-coated piezoelectric crystal biosensor. Sensors and Actuators B 1991, 3, 295-304.

88. Brownlee, M.; Cerami, A., A glucose-controlled insulin delivery system: semisynthetic insulin bound to lectin. Science 1979, 206, 1190-1191.

89. Seminoff, L. A.; Olsen, G. B.; Kim, S. W., A self-regulating insulin delivery system. I. Characterization of a synthetic glycosylated insulin derivative. Int. J. Pharm. 1989, 54, 241-249.

90. Kabilan, S.; Marshall, A. J.; Sartain, F. K.; Lee, M.-C.; Hussain, A.; Yang, X.; Blyth, J.; Karangu, N.; James, K.; Zeng, J.; Smith, D.; Domschke, A.; Lowe, C. R., Holographic glucose sensors. Biosensors and Bioelectronics 2005, 20, 1605-1610.

91. Horgan, A. M.; Marshall, A. J.; Kew, S. J.; Dean, K. E. S.; Creasey, C. D.; Kabilan, S., Crosslinking of phenylboronic acid receptors as a means of glucose selective holographic detection. Biosensors and Bioelectronics 2006, 21, 1838-1845.

92. Aoki, T.; Nagao, Y.; Sanui, K.; Ogata, N.; Kikuchi, A.; Sakurai, Y.; Kataoka, K.; Okano, T., Glucose-sensitive lower critical solution temperature changes of copolymers composed of N-Isopropylacrylamide and phenylboronic acid moieties. Polym. J. 1996, $28,(4), 371-343$.

93. Zhang, R.; Bowyer, A.; Eisenthal, R.; Hubble, J. In Demonstration of an intelligent hydrogel based diffraction grating, Smart structures and materials 2005: Smart sensor technology and measurement systems, San Diego, CA, 2005; SPIE-Int. Soc. Opt. Eng. USA: San Diego, CA, 2005; pp 254-64. 
94. Gonzalez, B. M.; Christie, G.; Davidson, C. A. B.; Blyth, J.; Lowe, C. R., Divalent metal ion-sensitive holographic sensors. Anal. Chim. Acta 2005, 528, 219-228.

95. Chen, H.-M.; Wang, W.-C.; Chen, S.-H., A metal-chelating piezoelectric sensor chip for direct detection and oriented immobilization of polyhis-tagged proteins. Biotechnology Prog. 2004, 20, (4), 1237-1244.

96. Holtz, J. H.; Asher, S. A., Polymerized colloidal crystal hydrogel films as intelligent chemical sensing materials. Nature 1997, 389, 829-832.

97. Zhang, Y.; Ji, H.-F.; Brown, G. M.; Thundat, T., Detection of CrO42- using a hydrogel swelling microcantilever sensor. Anal. Chem. 2003, 75, (18), 4773-4777.

98. Miyata, T.; Asami, N.; Uragami, T., A reversibly antigen-responsive hydrogel. Nature 1999, 399, 766-769.

99. Kim, J.; Singh, N.; Lyon, A. L., Label-free biosensing with hydrogel microlenses. Angew. Chem. Int. Ed. 2006, 45, 1445-1449.

100. Lu, Z.-R.; Kopeckova, P.; Kopecek, J., Antigen responsive hydrogels based on polymerizable antibody Fab' fragment. Macromol. Bioscience 2003, 3, 296-300.

101. Avramov, I. D.; Kurosawa, S.; Rapp, M.; Krawczak, P.; Radeva, E. I., Investigations on plasma polymer coated SAW and STW resonators for chemical gas sensing applications. IEEE Trans. Microwave Theory Tech. 2001, 49, (4), 827-837.

102. Kuckling, D.; Harmon, M. E.; Frank, C. W., Photo-cross-linkable PNIPAAm copolymers. 1. Synthesis and characterization of constrained temperature-responsive hydrogel layers. Macromol. 2002, 35, 6377-6383.

103. Liang, L.; Rieke, P., C. ; Liu, J.; Fryxell, G., E. ; Young, J., S. ; Engelhard, M., H. ; Alford, K., L., Surfaces with reversible hydrophilic/hydrophobic characteristics on cross-linked poly(N-Isopropylacrylamide) hydrogels. Langmuir 2000, 16, 8016-8023.

104. Hiratsuka, A.; Karube, I., Plasma polymerized films for sensor devices. Electroanal. 2000, 12, (9), 695-702. 
105. Partridge, A.; Harris, P.; Hirotsu, T.; Kurosawa, S., Plasma polymers applied to chemical sensing. Plasmas and polymers 2000, 5, (3/4), 191-200.

106. Kraus, F.; Cruz, S.; Muller, J., Plasma polymerized silicon organic thin films from HMDSN for capacitive humidity sensors. Sensors and Actuators B 2003, 88, 300-311.

107. Sadhir, R. K.; Sanjana, Z. N., Plasma deposited thin films suitable as moisture sensors. J. Mat. Sci. 1991, 26, (15), 4261-4267.

108. Bruno, P.; Cicala, G.; Corsi, F.; Dragone, A.; Losacco, A. M., High relative humidity range sensor based on polymer-coated STW resonant device. Sensors and Actuators B 2004, 100, 126-130.

109. Radeva, E., Thin plasma-polymerized layers of hexamethyldisiloxane for acoustoelectronic humidity sensors. Sensors and Actuators B 1997, 44, 275-278.

110. Georgieva, V.; Radeva, E.; Spassov, L., Ammonia sorptive properties of plasma polymer films obtained from hexamethyldisiloxane. Vacuum 2000, 58, 315-32-.

111. Da Silva, M. L. P.; Tan, I. H.; Filho, A. P. N.; Galeazzo, E.; Jesus, D. P., Use of plasma polymerized highly hydrophobic hexamethyldisilazane (HMDS) films for sensor development. Sensors and Actuators A 2003, 91, 362-369.

112. Inagaki, N.; Tasaka, S.; Nozue, Y., Plasma polymerization of metal acetylacetonates and application for gas sensor devices. J. Appl. Polym. Sci. 1992, 45, 1041-1048.

113. Sadhir, R. K.; Schoch Jr., K. F.; Wood, S., Plasma-polymerized metal phthalocyanine films: preparation, properties and morphology. Synth. Met. 1988, 26, 391402.

114. Kurosawa, S.; Tawara-Kondo, E.; Minoru, N.; Kamo, N., Detection of polycyclic compounds as mutagens using piezoelectric quartz crystal coated with plasmapolymerized phthalocyanine derivatives. Sensors and Actuators B 1997, 43, 175-179.

115. Hosono, K.; Matsubara, I.; Murayama, N.; Shin, W.; Izu, N., The sensitivity of 4ethylbenzenesulfonic acid-doped plasma polymerized polypyrrole films to volatile organic compounds. Thin Solid Films 2005, 484, 396-399. 
116. Giungato, P.; Ferrara, M. C.; Musio, F.; d'Agostino, R., Plasma polymerized thiophene for sensing volatile chemicals: synthesis, chemical characterization, and lowfrequency resistance measurements. Plasmas and polymers 1996, 1, (4), 283-297.

117. Akimoto, T.; Ikebukuro, K.; Karube, I., A surface plasmon resonance probe with a novel integrated reference sensor surface. Biosensors and Bioelectronics 2003, 18, 1447-1453.

118. Mayes, A. G., Immobilization chemistry. In Biomolecular sensors, Gizeli, E.; Lowe, C. R., Eds. Taylor and Francis: New York, NY, 2002; p 78.

119. Hiratsuka, A.; Mugurama, H.; Lee, K.-H.; Karube, I., Organic plasma process for simple and substrate-independent surface modification of polymeric BioMEMS devices. Biosensors and Bioelectronics 2004, 19, 1667-1672.

120. Hiratsuka, A.; Kojima, K.-i.; Mugurama, H.; Lee, K.-H.; Suzuki, H.; Karube, I., Electron transfer mediator micro-biosensor fabrication by organic plasma process. Biosensors and Bioelectronics 2005, 21, 957-964.

121. Mugurama, H.; Hiratsuka, A.; Karube, I., Thin-film glucose biosensor based on plasma-polymerized film: simple design for mass production. Anal. Chem. 2000, 72, 2671-2675.

122. Nakanishi, K.; Mugurama, H.; Karube, I., A novel method of immobilizing antibodies on a quartz crystal microbalance using plasma-polymerized films for immunosensors. Anal. chem. 1996, 68, 1695-1700.

123. Nakamura, R.; Mugurama, H.; Ikebukuro, K.; Sasaki, S.; Nagata, R.; Karube, I.; Pedersen, H., A plasma-polymerized film for surface plasmon resonance immunosensing. Anal. Chem. 1997, 69, 4649-4652.

124. Liu, B.; Yang, Y.-H.; Wu, Z.-Y.; Wang, H.; Shen, G.-L.; Yu, R.-Q., A potentiometric acetylcholinesterase biosensor based on plasma-polymerized film. Sensors and Actuators B 2005, 104, 186-190.

125. Wang, H.; Li, D.; Wu, Z.; Shen, G.; Yu, R., A reusable piezo-immunosensor with amplified sensitivity for ceruloplasmin based on plasma-polymerized film. Talanta 2004, 62, 201-208. 
126. Wang, H.; Liu, Y.; Tang, Y.; Deng, T.; Shen, G.; Yu, R., A protein-A based orientation-controlled immobilization strategy for antibodies using nanometer-sized gold particles and plasma-polymerized film. Anal. Biochem. 2004, 324, 219-224.

127. Wang, H.; Wang, C.; Lei, C.; Wu, Z.; Shen, G.; Yu, R., A novel biosensing interfacial design produced by assembling nano-Au particles on amine-terminated plasma-polymerized films. Anal. Bioanal. Chem. 2003, 377, 632-638.

128. Wang, H.; Wu, J.; Li, J.; Ding, Y.; Shen, G.; Yu, R., Nanogold particle-enhanced oriented adsorption of antibody fragments for immunosensing platforms. Biosensors and Bioelectronics 2005, 20, 2210-2217.

129. Peppas, N. A., Hydrogels in medicine and pharmacy. CRC Press: Boca Raton, FL, 1987.

130. Hilt, Z. J.; Gupta, A. K.; Bashir, R.; Peppas, N. A., Ultrasensitive Biomems Sensors Based on Microcantilevers Patterned with Environmentally Responsive Hydrogels. Biomed. Microdev. 2003, 5, (3), 177-184.

131. Harmon, M. E.; Kuckling, D.; Frank, C. W., Photo-cross-linkable PNIPAAm copolymers. 2. Effects of constraint on temperature and $\mathrm{pH}$-responsive hydrogel layers. Macromol. 2003, 36, 162-172.

132. Kuckling, D.; Hoffmann, J.; Plotner, M.; Ferse, D.; Kretschmer, K.; Adler, H.-J. P.; Arndt, K.-F.; Riechelt, R., Photo crosslinkable poly(N-Isopropylacrylamide) copolymers III: micro-fabricated temperature responsive hydrogel. Polymer 2003, 44, 4455-4462.

133. Lesho, M. J.; Sheppard Jr., N. F., Adhesion of polymer films to oxidized silicon and its effect on performance of a conductometric $\mathrm{pH}$ sensor. Sens. Actuator B 1996, 37, 61-66.

134. Cunliffe, D.; Alarcon, C. d. 1. H.; Peters, V.; Smith, J. R.; Alexander, C., Thermoresponsive surface-grafted Poly(N-Isopropylacrylamide) copolymers: Effect of phase transitions on protein and bacterial attachment. Langmuir 2003, 19, 2888-2899.

135. Liang, L.; Rieke, P. C.; Fryxell, G. E.; Liu, J.; Engehard, M. H.; Alford, K. L., Temperature-sensitive surfaces prepared by UV photografting reaction of photosensitizer and N-Isopropylacrylamide. J. Phys. Chem. B 2000, 104, 11667-11673. 
136. Lin, S.-Y.; Chen, K.-S.; Liang, R.-C., Thermal micro ATR/FTIR spectroscopic system for quantitative study of the molecular structure of poly(N-Isopropylacrylamide) in water. Polymer 1999, 40, 2619.

137. Skrovanek, D. J.; Painter, P. C.; Coleman, M. M., Hydrogen bonding in polymers. 2. Infrared temperature studies of Nylon 11. Macromol. 1986, 19, 699-705.

138. Percot, A.; Zhu, X. X.; Lafleur, M., A simple FTIR spectroscopic method for the determination of the lower critical solution temperature of N-Isopropylacrylamide copolymers and related hydrogels. J. Polym. Sci. B 2000, 38, 907-915.

139. Ramon, O.; Kesselman, E.; Berkovici, R.; Cohen, Y.; Paz, Y., Attenuated total reflectance/ Fourier transform infrared studies on the phase-separation process of aqueous solutions of Poly(N-Isopropylacrylamide). J. Polym. Sci. B 2001, 39, 1665-1677.

140. Maeda, Y.; Higuchi, T.; Ikeda, I., Change in hydration state during the coilglobule transition of aqueous solutions of Poly (N-Isopropylacrylamide) as evidenced by FTIR spectroscopy. Langmuir 2000, 16, 7503-7509.

141. Takei, Y., G.; Aoki, T.; Sanui, K.; Ogata, N.; Sakurai, Y.; Okano, T., Dynamic contact angle measurement of temperature-responsive surface properties for Poly(NIsopropylacrylamide) grafted surfaces. Macromol. 1994, 27, 6163-6166.

142. Wu, C.; Zhou, S., Volume phase transition of swollen gels: Discontinuous or continuous. Macromol. 1997, 30, 574-576.

143. Schmaljohann, D.; Beyerlein, D.; Nitschke, M.; Werner, C., Thermo-reversible swelling of thin hydrogel films immobilized by low-pressure plasma. Langmuir 2004, 20, (23), 10107-10114.

144. Zhang, J.; Pelton, R.; Deng, Y., Temperature dependent contact angles of water on poly(N-Isopropylacrylamide) gels. Langmuir 1995, 11, 2301-2302.

145. Volpe, C. D.; Cassinelli, C.; Morra, M., Wilhelmy plate measurements on Poly(N-Isopropylacrylamide)-grafted surfaces. Langmuir 1998, 14, 4650-4656.

146. Sauerbrey, G., Z. Phys. 1959, 155, 206-222. 
147. Nomura, T.; Okuhara, M., Frequency shifts of piezoelectric quartz crystals immersed in organic liquids. Anal. Chim. Acta 1982, 142, 281-284.

148. Hillman, R. A., The electrochemical quartz crystal microbalance. In Encyclopedia of Electrochemistry, Bard, A. J.; Stratmann, M., Eds. Wiley-VCH Verlag GmbH \& Co. KGaA: Weinheim, Germany, 2003; Vol. 3, pp 230-289.

149. Janshoff, A.; Galla, H.-J.; Steinem, C., Piezoelectric mass-sensing devices as biosensors - An alternative to optical biosensors? Angew. Chem. Int. Ed. 2000, 39, 40044032.

150. Rodahl, M.; Hook, F.; Krozer, A.; Brzezinski, P.; Kasemo, B., Quartz crystal microbalance setup for frequency and Q-factor measurements in gaseous and liquid enviroments. Rev. Sci. Instr. 1995, 66, (7), 3924-3930.

151. Vogt, B. D.; Soles, C. L.; Lee, H.-J.; Lin, E. K.; Wu, W.-1., Moisture absorption into ultrathin hydrophilic polymer films on different substate surfaces. Polymer 2005, 46, (5), 1635-1642.

152. Vogt, B. D.; Soles, C. L.; Lee, H.-J.; Lin, E. K.; Wu, W.-1., Moisture absorption and absorption kinetics in polyelectrolyte films: Influence of film thickness. Langmuir 2004, 20, 1453-1458.

153. Asberg, P.; Bjork, P.; Hook, F.; Inganas, O., Hydrogels from a water-soluble zwitterionic polythiophene: Dynamics under $\mathrm{pH}$ change and biomolecular interactions observed using quartz crystal microbalance with dissipation monitoring. Langmuir 2005, 21, 7292-7298.

154. Irwin, E. F.; Ho, J. E.; Kane, S. R.; Healy, K. E., Analysis of interpenetrating polymer networks via quartz crystal microbalance with dissipation monitoring. Langmuir 2005, 21, 5529-5536.

155. Falt, S.; Wagberg, L.; Vesterlind, E.-L., Swelling of model films of cellulose having different charge densities and comparison to the swelling behavior of corresponding fibers. Langmuir 2003, 19, 7895-7903.

156. Plunkett, M. A.; Wang, Z.; Rutland, M. W.; Johannsmann, D., Adsorption of pNIPAM layers on hydrophobic gold surfaces, measured in situ by QCM and SPR. Langmuir 2003, 19, 6837-6844. 
157. Zhang, G., Study on conformation change of thermally sensitive linear grafted Poly(N-Isopropylacrylamide) chains by quartz crystal microbalance. Macromol. 2004, 37, 6553-6557.

158. Liu, G.; Zhang, G., Collapse and swelling of thermally sensitive Poly(NIsopropylacrylamide) brushes monitored with a quartz crystal microbalance. J. Phys. Chem. B 2005, 109, 743-747.

159. Liu, G.; Zhang, G., Reentrant behavior of Poly(N-Isopropylacrylamide) brushes in water-methanol mixtures investigated with a quartz crystal microbalance. Langmuir 2005, 21, 2086-2090.

160. Radeva, E. I.; Avramov, I. D., High-resolution humidity measurements with surface transverse wave based resonant devices. Applications to wireless remote sensing. Mat. Sci. Eng. C 2000, 12, 71-76.

161. Munro, J. C.; Frank, C. W., Polyacrylamide Adsorption from Aqueous Solutions on Gold and Silver Surfaces Monitored by the Quartz Crystal Microbalance. Macromol. 2004, 37, 925.

162. Hook, F.; Rodahl, M.; Bengt, K.; Brzezinski, P., Structural changes in hemoglobin during adsorption to solid surfaces: Effect of $\mathrm{pH}$, ionic strength, and ligand binding. Proc. Natl. Acad. Sci. USA 1998, 95, 12271-12276.

163. Kanazawa, K. K.; Gordon II, J. G., Frequency of a quartz microbalance in contact with liquid. Anal. Chem. 1985, 57, 1770-1771.

164. Rodahl, M.; Kasemo, B., On the measurement of thin liquid overlayers with the quartz-crystal microbalance. Sens. Actuators, A 1996, 54, 448-456.

165. Zhulina, E. B.; Borisov, O. V.; Pryamitsyn, V. A.; Birshtein, T. M., Coil-globule type transitions in polymers. 1. Collapse of layers of grafted polymer chains. Macromol. 1991, 24, 140-149.

166. Scott, R. A.; Cowans, B. A.; Peppas, N. A., NMR Spectroscopy and free volume analysis of the effects of copolymer composition on the swelling kinetics and chain dynamics of highly crosslinked copolymers of acrylic acid with PEG-containing multiacrylates. J. Polym. Sci. B: Polym. Phys. 1999, 37, 1953-1968. 
167. De Wilde, W. P.; Shopov, P. J., A simple model for moisture sorption in epoxies with sigmoidal and two-stage sorption effects. Composite Struct. 1994, 27, 243-252.

168. Long, F. A.; Richman, D., Concentration gradients for diffusion of vapors in glassy polymers and their relation to time dependent diffusion phenomena. J. Am. Chem. Soc. 1960, 82, 513-519.

169. van der Wel, G. K.; Adan, O. C. G., Moisture in organic coatings - a review. Prog. Org. Coatings 1999, 37, 1-14.

170. Devine, D. M.; Higginbotham, C. L., Synthesis and characterisation of chemically crosslinked N-vinyl pyrrolidinone (NVP) based hydrogels. Eur. Polym. J. 2005, 41, 1272-1279.

171. Barbucci, R.; Magnani, A.; Consumi, M., Swelling Behavior of Carboxymethylcellulose Hydrogels in Relation to Cross-Linking, $\mathrm{pH}$, and Charge Density. Macromol. 2000, 33, 7475-7480.

172. Gemmei-Ide, M.; Motonaga, T.; Kitano, H., State of irremovable water in solid polymer films examined by Fourier transform infrared spectroscopy I: Poly(Ethylene glycol) dimethyl ether. Langmuir 2006, 22, 2422-2425.

173. Gerbsch, N.; Buchholz, R., New processes and actual trends in biotechnology. FEMS Microbiol. Rev. 1995, 16, 259-269.

174. Avnir, D.; Coradin, T.; Lev, O.; Livage, J., Recent bio-applications of sol-gel materials. J. Mater. Chem. 2006, 16, 1013-1030.

175. Cosnier, S., Affinity biosensors based on electropolymerized films. Electroanal. 2005, 17, (19), 1701-1715.

176. Guschin, D.; Yershov, G.; Zaslavsky, A.; Gemmell, A.; Shick, V.; Proudnikov, D.; Arenkov, P.; Mirzabekov, A., Manual manufacturing of oligonucleotide, DNA, and protein microchips. Anal. Biochem. 1997, 250, 203-211.

177. Charles, P. T.; Taitt, C. R.; Goldman, E. R.; Rangasammy, J. G.; Stenger, D. A., Immobilization strategy and characterization of hydrogel-based thin films for interrogation of ligand binding with staphylococcal enterotoxin $B$ (SEB) in a protein microarray format. Langmuir 2004, 20, 270-272. 
178. Lee, Y.-S.; Mrksich, M., Protein chips: from concept to practice. TIBITECH 2002, 20, (12), S14-S18.

179. Kusnezow, W.; Hoheisel, J. D., Antibody microarrays: Promises and problems. BioTechniques 2002, 33, S14-S23.

180. Charles, P. T.; Goldman, E. R.; Rangasammy, J. G.; Schauer, C. L.; Chen, M.-S.; Taitt, C. R., Fabrication and characterization of 3D hydrogel microarrays to measure antigenicity and antibody functionality for biosensor applications. Biosensors and Bioelectronics 2004, 20, 753-764.

181. Rezvin, A. R., Ryan, J.; Yadavalli, Vamsi, K.; Koh, Won-Gun; Deister, Curt; Hile, David, D.; Mellott, Michael, B. and Pishko, Michael, V., Fabrication of poly(ethylene glycol) hydrogel microstructures using photolithography. Langmuir 2001, $17,5440-5447$.

182. Heo, J.; Crooks, R. M., Microfluidic biosensor based on an array of hydrogeltrapped enzymes. Anal. Chem. 2005, 77, 6843-6851.

183. Bange, A.; Halsall, H. B.; Heineman, W. R., Microfluidic immunosensor systems. Biosensors and Bioelectronics 2005, 20, 2488-2503.

184. Lim, J.-H.; Ginger, D. S.; Lee, K.-B.; Heo, J.; Nam, J.-M.; Mirkin, C. A., Directwrite dip-pen nanolithography of proteins on modified silicon oxide surfaces. Angew. Chem. Int. Ed. 2003, 115, (2411-2414).

185. Kurzawa, C.; Hengstenberg, A.; Schuhmann, W., Immobilization method for the preparation of biosensors based on $\mathrm{pH}$ shift-induced deposition of biomoleculecontaining polymer films. Anal. Chem. 2002, 74, 355-361.

186. Van der Biest, O. O.; Vandeperre, L. J., Electrophoretic deposition of materials. Annu. Rev. Mater. Sci. 1999, 29, 327-352.

187. Kalia, Y. N.; Naik, A.; Garrison, J.; Guy, R., Iontophoretic drug delivery. Adv. Drug Delivery Rev. 2004, 56, 619-658.

188. Shalaby, W. S.; Abdallah, A. A.; Park, H.; Park, K., Loading of bovine serum albumin into hydrogels by an electrophoretic process and its potential application to protein drugs. Pharm. Res. 1993, 10, (3), 457-460. 
189. Stubbs, D. D. Development of an acoustic wave based biosensor for vapor phase detection of small molecules. Georgia Institute of Technology, Atlanta, GA, 2005.

190. Cavic, B. A.; Hayward, G. L.; Thompson, M., Acoustic waves and the study of biochemical macromolecules and cells at the sensor-liquid interface. The Analyst 1999, $124,1405-1420$.

191. Stubbs, D. D.; Hunt, W. D.; Lee, S. H.; Doyle, D. F., Gas phase activity of antiFITC antibodies immobilized on a surface acoustic wave resonator device. Biosens. Bioelectron. 2002, 17, (6-7), 471-477.

192. Lee, S.-H.; Stubbs, D. D.; Cairney, J.; Hunt, W. D., Rapid detection of bacterial spores using a quartz crystal microbalance (QCM) immunoassay. IEEE Sensors J. 2005, $5,(4), 737-743$.

193. Thompson, M.; Arthur, C. L.; Dhaliwal, G. K., Liquid-phase piezoelectric and acoustic transmission studies of interfacial immunochemistry. Anal. Chem. 1986, 58, 1206-1209.

194. Marxer, C. G.; Coen, M. C.; Greber, T.; Greber, U. F.; Schlapbach, L., Cell spreading on quartz crystal microbalance elicits positive frequency shifts indicative of viscosity changes. Anal. Bioanal. Chem. 2003, 377, 578-586.

195. Hunt, W. D.; Stubbs, D. D.; Lee, S. H., Time-dependent signatures of acoustic wave biosensors. Proceedings of the IEEE 2003, 91, (6), 890-901.

196. Martin, S. J.; Frye, G. C.; Senturia, S. D., Dynamics and response of polymercoated surface acoustic wave devices: effect of viscoelastic properties and film resonance. Anal. Chem. 1994, 14, 2201-2219.

197. Grate, J. W.; Klusty, M.; McGill, A. R.; Abraham, M. H.; Whiting, G.; AndonianHaftvan, J., The predominant role of swelling-induced modulus changes of the sorbent phase in determining the responses of polymer-coated surface acoustic wave sensors. Anal. Chem. 1994, 64, 610-624.

198. Buttry, D. A.; Ward, M. D., Measurement of interfacial processes at electrode surfaces with the electrochemical quartz crystal microbalance. Chem. Rev. 1992, 92, 1355-1379. 
199. Lucklum, R.; Behling, C.; Hauptmann, P., Role of mass accumulation and viscoelastic film properties for the response of acoustic-wave-based chemical sensors. Anal. Chem. 1999, 71, 2488-2496.

200. Martin, S. J.; Frye, G. C., Surface acoustic wave response to changes in viscoelastic film properties. Appl. Phys. Lett. 1990, 57, 1867-1869.

201. Grate, J. W.; Wenzel, S. W.; White, R. M., Frequency-independent and frequency-dependent polymer transitions observed on flexural plate wave ultrasonic sensors. Anal. Chem. 1992, 64, 413-423.

202. Teuscher, J. H.; Yeager, L. J.; Yoo, H.; Chadwick, J. E.; Garrell, R. L., Phase transitions in thin alkane films and alkanethiolate monolayers on gold detected with a thickness shear mode device. Faraday Discuss. 1997, 107, 399-416.

203. Ricco, A. J.; Staton, A. W.; Crooks, R. M.; Kim, T., Single-monolayer insitu modulus measurements using a SAW device photocrosslinking of a diacetylenic thiolbased monolayer. Faraday Discuss. 1997, 107, 247-258.

204. Schultz, P. J.; Lynn, K. G., Interaction of positron beams with surfaces, thin films, and interfaces. Rev. Mod. Phys. 1988, 60, (3), 701-779.

205. Jean, Y. C., Comments on the paper "Can positron annihilation lifetime spectroscopy measure the free-volume hole size distribution in amorphous polymers?" Macromol. 1996, 29, 5756-5757.

206. Jean, Y. C.; Zhang, J.; Chen, H.; Li, Y.; Liu, G., Positron annihilation spectroscopy for surface and interface studies in nanoscale polymeric films. Spectrochim. Acta A 2005, 61, 1683-1691.

207. Mallon, P. E., Application to polymers. In Principles and applications of positron and positronium chemistry, Jean, Y. C.; Mallon, P. E.; Schrader, D. M., Eds. World Scientific: Singapore, 2003; pp 253-277.

208. Jean, Y. C.; Mallon, P. E.; Zhang, R.; Chen, H.; Wu, Y. C.; Li, Y.; Zhang, J., Applications of slow positrons to polymeric surfaces and coatings. In Principles and applications of positron and positronium chemistry, Jean, Y. C.; Mallon, P. E.; Schrader, D. M., Eds. World Scientific: Singapore, 2003. 
209. Jean, Y. C.; Zhang, R.; Cao, H.; Yuan, J.-P.; Huang, C.-M.; Nielsen, B.; AsokaKumar, P., Glass transition of polystyrene near the surface studied by slow-positronannihilation spectroscopy. Phys. Rev. B 1997, 56, (14), R8459-RR8462.

210. Cao, H.; Zhang, R.; Sundar, C. S.; Yuan, J.-P.; He, Y.; Sandreczki, T. C.; Jean, Y. C., Degradation of polymer coating systems studied by positron annihilation spectroscopy. 1. UV irradiation effect. Macromol. 1998, 31, 6627-6635.

211. Zhang, R.; Gu, X.; Chen, H.; Zhang, J.; Li, Y.; Nguyen, T.; Sandreczki, T. C.; Jean, Y. C., Study of the photodegradation of epoxy polymers with slow positron annihilation spectroscopy. J. Polym. Sci. Part B: Polym. Phys. 2004, 42, 2441-2459.

212. Zhang, R.; Mallon, P. E.; Chen, H.; Huang, C. M.; Zhang, J.; Li, Y.; Wu, Y.; Sandreczki, T. C.; Jean, Y. C., Characterization of photodegradation of a polyurethane coating by positron annihilation spectroscopy: correlation with crosslink density. Prog. Org. Coat. 2001, 42, 244-252.

213. Jean, Y. C.; Chen, H.; Zhang, R.; Li, Y.; Zhang, J., Early stage of deterioration in polymeric coatings detected by positron annihilation spectroscopy. Prog. Org. Coat. 2005, 52, 1-8.

214. Petkov, M. P.; Weber, M. H.; Lynn, K. G.; Rodbell, K. P.; Cohen, S. A., Doppler broadening positron annihilation spectroscopy: A technique for measuring open-volume defects in silsesquioxane spin-on glass films. Appl. Phys. Lett. 1999, 74, (15), 2146-2148.

215. Sun, J.-N.; Gidley, D. W.; Frieze, W. E.; Ryan, E. T., Depth-profiling plasmainduced densification of porous low-k thin films using positronium annihilation lifetime spectroscopy. Appl. Phys. Lett. 2002, 81, (8), 1447-1449.

216. Myneni, S.; Peng, H.-G.; Gidley, D. W.; Hess, D. W., Compatibility of high pressure cleaning mixtures with a porous low dielectric constant film: A positronium annihilation lifetime spectroscopic study. J. Vac. Sci. Technol. B 2005, 23, (4), 14631469.

217. Li, Y.; Zhang, R.; Chen, H.; Zhang, J.; Suzuki, R.; Ohdaira, T.; Feldstein, M. M.; Jean, Y. C., Depth profile of free volume in a mixture and copolymers of poly(N-vinylpyrrolidone) and poly(ethylene glycol) studied by positron annihilation spectroscopy. Biomacromol. 2003, 4, 1856-1864. 
218. Panda, A.; Sodaye, H. S.; Acharya, R. N.; Goswami, A.; Pujari, P. K.; Sabharwal, S.; Manohar, S. B., Positron annihilation studies on radiation-crosslinked poly(NIsopropylacrylamide) hydrogels. J. Polym. Sci. Part A: Polym. Chem. 2000, 38, 34623466.

219. Sousa, R. G.; Freitas, R. F. S.; Magalhaes, W. F., Structural characterization of poly(N-Isopropylacrylamide) gels and some of their copolymers with acrylamide through positron annihilation lifetime spectroscopy. Polymer 1998, 39, (16), 3815-3819.

220. Hsueh, C.-H.; Lee, S.; Lin, H.-Y., Analyses of mode I edge delamination by thermal stresses in multilayer systems. Composites: Part B 2006, 37, 1-9.

221. Yu, H. H.; He, M. Y.; Hutchinson, J. W., Edge effects in thin film delamination. Acta Mater. 2001, 49, 93-107.

222. Hsueh, C. H., Thermal stresses in elastic multilayer systems. Thin Solid Films 2002, 418, 182-188.

223. Xie, W.; Sitaraman, S. K., Interfacial thermal stress analysis of anisotropic multilayered electronic packaging structures. J. Electron. Packag. 2000, 122, 61-66. 